Prepared for the U.S. Department of Energy

under Contract DE-AC05-76RL01830

\title{
Methods and Instruments for Fast Neutron Detection
}
DV Jordan
KR McCormick
PL Reeder
AJ Peurrung
M Cooper
GA Warren

May 2005

\section{Pacific Northwest}

NATIONAL LABORATORY

Proudly Operated by Battelle Since 1965 


\title{
DISCLAIMER
}

This report was prepared as an account of work sponsored by an agency of the United States Government. Neither the United States Government nor any agency thereof, nor Battelle Memorial Institute, nor any of their employees, makes any warranty, express or implied, or assumes any legal liability or responsibility for the accuracy, completeness, or usefulness of any information, apparatus, product, or process disclosed, or represents that its use would not infringe privately owned rights. Reference herein to any specific commercial product, process, or service by trade name, trademark, manufacturer, or otherwise does not necessarily constitute or imply its endorsement, recommendation, or favoring by the United States Government or any agency thereof, or Battelle Memorial Institute. The views and opinions of authors expressed herein do not necessarily state or reflect those of the United States Government or any agency thereof.

\author{
PACIFIC NORTHWEST NATIONAL LABORATORY \\ operated by \\ BATTELLE \\ for the \\ UNITED STATES DEPARTMENT OF ENERGY \\ under Contract DE-AC05-76RL01830
}

Printed in the United States of America
Available to DOE and DOE contractors from the Office of Scientific and Technical Information,
P.O. Box 62, Oak Ridge, TN 37831-0062;
ph: (865) 576-8401
fax: $(865)$ 576-5728
email: reports@adonis.osti.gov

\begin{abstract}
Available to the public from the National Technical Information Service, U.S. Department of Commerce, 5285 Port Royal Rd., Springfield, VA 22161 ph: (800) 553-6847 fax: $(703) 605-6900$ email: orders@ntis.fedworld.gov online ordering: http://www.ntis.gov/ordering.htm
\end{abstract}

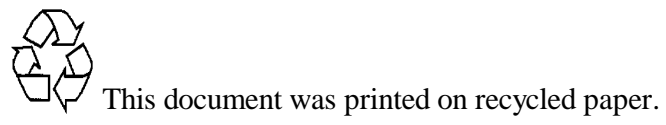




\section{Methods and Instruments for Fast Neutron Detection}

D.V. Jordan

P.L. Reeder

M. Cooper

K.R. McCormick

A.J. Peurrung

G.A. Warren

May 23, 2005

Prepared for the U.S. Department of Energy under Contract DE-AC05-76RL01830

Pacific Northwest National Laboratory

Richland, Washington 99352 


\section{Abstract}

Pacific Northwest National Laboratory evaluated the performance of a large-area $\left(\sim 0.7 \mathrm{~m}^{2}\right)$ plastic scintillator time-of-flight (TOF) sensor for direct detection of fast neutrons. This type of sensor is a readily area-scalable technology that provides broad-area geometrical coverage at a reasonably low cost. It can yield intrinsic detection efficiencies that compare favorably with moderator-based detection methods. The timing resolution achievable should permit substantially more precise time windowing of return neutron flux than would otherwise be possible with moderated detectors. The energy-deposition threshold imposed on each scintillator contributing to the event-definition trigger in a TOF system can be set to blind the sensor to direct emission from the neutron generator. The primary technical challenge addressed in the project was to understand the capabilities of a neutron TOF sensor in the limit of large scintillator area and small scintillator separation, a size regime in which the neutral particle's flight path between the two scintillators is not tightly constrained.

The project comprised an experimental campaign and a modeling campaign. The experimental campaign focused on measuring the response of an existing, dual-sheet scintillator sensor to mono-energetic gamma sources (including ${ }^{137} \mathrm{Cs}$ and ${ }^{54} \mathrm{Mn}$ ) and a ${ }^{252} \mathrm{Cf}$ gamma + neutron source. The sensor's intrinsic gamma and neutron detection efficiencies were mapped as a function of the TOF threshold above which the sensor's response is integrated. A fast neutron intrinsic detection efficiency of approximately $2.5 \%$, averaged over the ${ }^{252} \mathrm{Cf}$ neutron spectrum, was obtained at a TOF threshold that permits approximately 10,000:1 gamma rejection. An additional contribution to the sensor's total intrinsic neutron detection efficiency of roughly $1.3 \%$ results from including the TOF response to neutron flux reflected from a 5.08 $\mathrm{cm}$ ( 2 in.) layer of $\mathrm{Pb}$ shielding surrounding the sensor on four sides. In addition to the TOF measurements, the report presents preliminary investigations of the supplementary neutron spectroscopic information content available in scintillator pulse-height measurements.

The modeling campaign consisted of development of a simulation code based upon the Geant 4 radiation transport framework. A prescription for calculating effective particle interaction times in each scintillator of the TOF sensor is described. This prescription avoids the significant computational overhead associated with tracking large ensembles of scintillation photons, at the cost of sacrificing a realistic model of the sensor's photomultiplier tube (PMT) signal pulse development with time. The model predicts the qualitative features of the neutron TOF distribution (although it underpredicts the $\mathrm{Pb}$ reflected component) and provides a consistent description of the measured neutron and gamma detection efficiencies. The model fails to predict the shapes of the gamma and neutron responses accurately enough to permit reliable prediction of the details of the gamma rejection as a function of TOF threshold.

The report concludes by comparing the intrinsic neutron detection efficiency of the present TOF sensor to a Monte Carlo N-Particle Transport Code (MCNP) simulation of a hypothetical, moderator-based ${ }^{3} \mathrm{He}$ tube sensor of comparable area. It is demonstrated that the TOF method yields a fast neutron intrinsic detection efficiency that compares favorably with the calculated efficiency of the moderator-based sensor. Although the simple design assumed for the hypothetical ${ }^{3} \mathrm{He}$ sensor has not been subjected to performance optimization in any of its geometrical parameters, neither is the design completely unrealistic. Thus, the comparison serves the purpose of highlighting the potential competitive position of the TOF method, especially in view of the cost benefits realizable from deploying plastic scintillator in a large-area sensor. 



\section{Executive Summary}

The scope of this sub-task of Pacific Northwest National Laboratory's (PNNL's) contribution to the Department of Homeland Security (DHS) Science and Technology (S\&T) Rad/Nuc Countermeasures Active Sensors project is to evaluate the performance of a large-area $\left(\sim 0.7 \mathrm{~m}^{2}\right)$, two-sheet plastic scintillator neutron time-of-flight (n-TOF) sensor for direct detection of fast neutrons, in support of Lawrence Livermore National Laboratory's (LLNL's) Active Detection program. A plastic scintillator nTOF sensor detects fast neutrons via successive elastic scatterings in two physically separated scintillation detectors. Measuring the particle flight time through the sensor, i.e., the time interval between correlated energy deposition "hits" in the two scintillators, permits discrimination between neutrons and gammas. A large-area scintillator sensor promises several attractive benefits as the enabling fast neutron detection technology in an active neutron interrogation program:

- Monolithic plastic scintillators represent a readily area-scaleable technology that provides broadarea geometrical coverage at reasonably low cost per unit area.

- The physical mechanism for neutron detection by the TOF method can yield intrinsic detection efficiencies that compare favorably with moderator-based detection methods. Coupled with large solid angle coverage at low cost per area, good intrinsic detection efficiency yields an economical sensor with high absolute neutron detection efficiency.

- The timing resolution achievable with a plastic scintillator-based system (of order $100 \mathrm{~ns}$ ) should permit substantially more precise time windowing of return neutron flux than would otherwise be possible with moderated detectors, such as ${ }^{3} \mathrm{He}$ tubes, where the time-scale for moderation is typically 100 times longer (10 microseconds). High-resolution time windowing can provide a powerful tool for background suppression in an active system in which the sensor response can be phase-locked to the generator of the interrogating radiation.

- The energy-deposition threshold imposed on each scintillator contributing to the event-definition trigger in a TOF system can be set to blind the sensor to direct emission from the neutron generator. This is particularly true in the case of the proposed LLNL "near-passive" system in which the interrogation beam consists of $60 \mathrm{keV}$ neutrons.

The primary technical challenge addressed in the project was to understand the capabilities (including gamma and fast neutron detection efficiencies, and the effectiveness of neutron/gamma discrimination) of an n-TOF sensor in the limit of large scintillator area, a size regime in which the neutral particle's flight path between the two scintillators is not tightly constrained.

The project comprised an experimental campaign and a modeling campaign. The experimental campaign leveraged an existing apparatus at PNNL, a dual-sheet plastic scintillator sensor (the so-called "ALPS II," in which the acronym stands for the NA-22 funded "Advanced Large-Area Plastic Scintillator" project at PNNL) designed for gamma detection portal monitor studies. The sensor consists of two aluminizedmylar wrapped sheets of $127 \mathrm{~cm} \times 57.2 \mathrm{~cm} \times 5.0 \mathrm{~cm}(50$ in. $\times 22.5$ in. $\times 2$ in.) BC-408 scintillator, separated by a center-to-center distance of $17.8 \mathrm{~cm}$ (7 in.). The shorter ends of both scintillator sheets are outfitted with three $12.7 \mathrm{~cm}$ (5 in.) diameter Hamamatsu photomultiplier tubes (PMTs) apiece, for a total of 12 PMTs for the entire sensor. The sensor is housed in a steel cabinet lined on four sides (sides, back, and bottom) with $5.08 \mathrm{~cm}$ ( 2 in.) thick $\mathrm{Pb}$. Note that the original purpose of the $\mathrm{Pb}$ lining was to reduce 
terrestrial gamma background in the sensor's use as a gamma portal monitor and is not necessarily an essential element in the application of the apparatus to TOF-based neutron detection. (However, the $\mathrm{Pb}$ lining was found to increase the sensor's total neutron response via "reflection" of neutrons back into the sensor that might otherwise have failed to scatter in both scintillators.) The ALPS II sensor's pulseprocessing and readout electronics, which include a Time-to-Digital Conversion (TDC) module, were reconfigured to emphasize measurements of timing information from only four of these PMTs, one at the center of each scintillator end. The sensor's data-acquisition and analysis software were tailored to calculate the transit time between the first and second scintillators for events in which all four participating PMTs presented valid pulses to the readout electronics in coincidence.

The sensor's mono-energetic gamma response and its fast neutron response were measured using ${ }^{137} \mathrm{Cs}$ (emitting a $662 \mathrm{keV}$ gamma) and ${ }^{252} \mathrm{Cf}$ (emitting both fast neutrons and gammas) point sources, respectively. Figure S.1 displays the TOF spectra for a particular value of the PMT discriminator threshold in the pulse-processing electronics $(10 \mathrm{mV})$. The gamma distribution is peaked at small TOF, consistent with their maximal velocity. The more slowly moving neutrons, in contrast, populate larger absolute values of TOF. The bimodal distribution arises from neutrons reflecting off the $\mathrm{Pb}$ shielding lining the sensor housing. Events in the positive-TOF "lobe" of the distribution correspond to neutrons traversing the sensor from front to back (i.e., in the order that normally-incident source neutrons would encounter the scintillators) whereas the negative-TOF lobe corresponds to neutrons elastically scattered off the rear $\mathrm{Pb}$ shielding wall that traverse the sensor from back to front. The reflection effect is much less important for the gamma source, and at the small (in absolute value) TOF values characteristic of the gamma peak, a reverse-direction distribution cannot be resolved from the front-to-back distribution. Note that the difference in the gamma and neutron TOF distributions provides the key to discriminating between the two particle types. A threshold or gate on the TOF distribution (imposed, for example, in the data-acquisition software) can be used to select the fast neutron portion of the spectrum, with the following performance trade-off in effect: As the TOF threshold is increased, the gamma rejection improves, but at the cost of lower neutron detection efficiency. Optimizing the performance of a TOF sensor within a particular operating environment typically involves selecting the TOF threshold to maximize neutron efficiency while simultaneously retaining satisfactory gamma rejection.

The sensor's intrinsic detection efficiency, i.e., the absolute detection efficiency expressed relative to the number of particles incident upon the front scintillator, is displayed in Figure $\mathrm{S} 2$ for the $10-\mathrm{mV}$ discriminator threshold. The efficiency is plotted as a function of the threshold TOF value above which the sensor's TOF distribution is integrated. The response is separated into "Forward" (i.e., front-to-back) and "Reverse" (back-to-front) components for both particle types, although the distinction is most meaningful for the bimodal neutron distribution. (For the unimodal gamma distribution, the distinction merely serves to quantify the asymmetry in the peak shape relative to the most probable TOF value.) The performance tradeoff discussed above can be observed in this figure. As the TOF threshold increases above $10 \mathrm{~ns}$, the ratio of the gamma efficiency to the neutron efficiency drops rapidly, indicating greater gamma rejection for a sensor "neutron" signal defined by this TOF threshold. But the neutron detection efficiency begins to decrease precipitously above about $20 \mathrm{~ns}$, consistent with the exclusion of valid neutron events (clearly evident in Figure S1) in the 10- to 20-ns portion of the spectrum. 


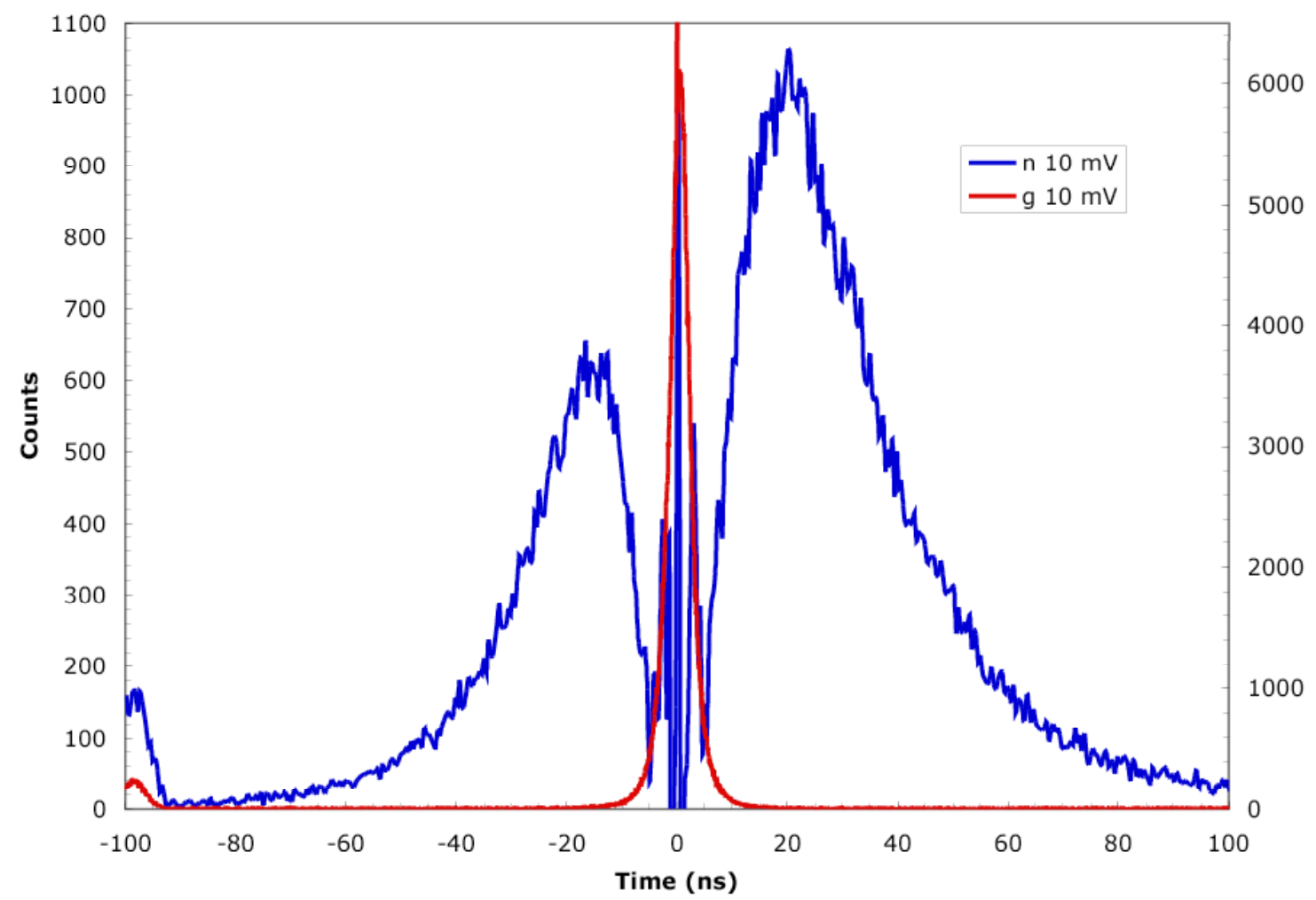

Figure S1. Measured TOF Response of the $0.7 \mathrm{~m}^{2}$, Dual-Scintillator Sheet Sensor. The sensor's TOF distribution for a mono-energetic gamma source $\left({ }^{137} \mathrm{Cs}, 662 \mathrm{keV}\right.$, red curve) is superimposed on the neutron-only TOF distribution measured with a ${ }^{252} \mathrm{Cf}$ source (blue curve). A portion of the ${ }^{252} \mathrm{Cf}$ TOF spectrum corresponding to the gamma emissions from this source has been subtracted to isolate the shape of the neutron distribution.

The results depicted in these two figures are typical of a wider set of data recorded at a variety of discriminator thresholds and detailed in the body of the report. At the beginning of the experimental campaign, it was anticipated that the sensor's response to a broader set of standard fast neutron sources (including, for example, $\mathrm{AmBe}, \mathrm{PuBe}$, and $\mathrm{PuO}_{2}$ ) would be measured in addition to ${ }^{252} \mathrm{Cf}$. These measurements would have provided an empirical basis for quantifying the potential use of a large-area, n-TOF sensor for differentiating (or classifying) fast neutron energy spectra based upon the shape of the TOF distribution. Unfortunately, PNNL facility use restrictions on these additional source types in the building housing the sensor precluded completing these measurements before the end of the project. The body of the report also describes a secondary aspect of the experimental campaign in which the TOF information was supplemented with measurements of the energy deposition in each scintillator, as registered in analog-to-digital converters (ADCs). These investigations attempted to address the issue of extracting spectroscopic information from the incident neutron flux, based upon the energy distribution of recoil protons in the scintillators. Without a more extensive variety of neutron source spectra measurements, however, the enhancement of the neutron spectroscopic differentiation capability already inherent in the shape of the TOF distribution alone could not be definitively quantified in this secondary campaign. The results in that section of the report should be considered of a more exploratory and preliminary character than the TOF-only campaign that represents the main thrust of the project. 


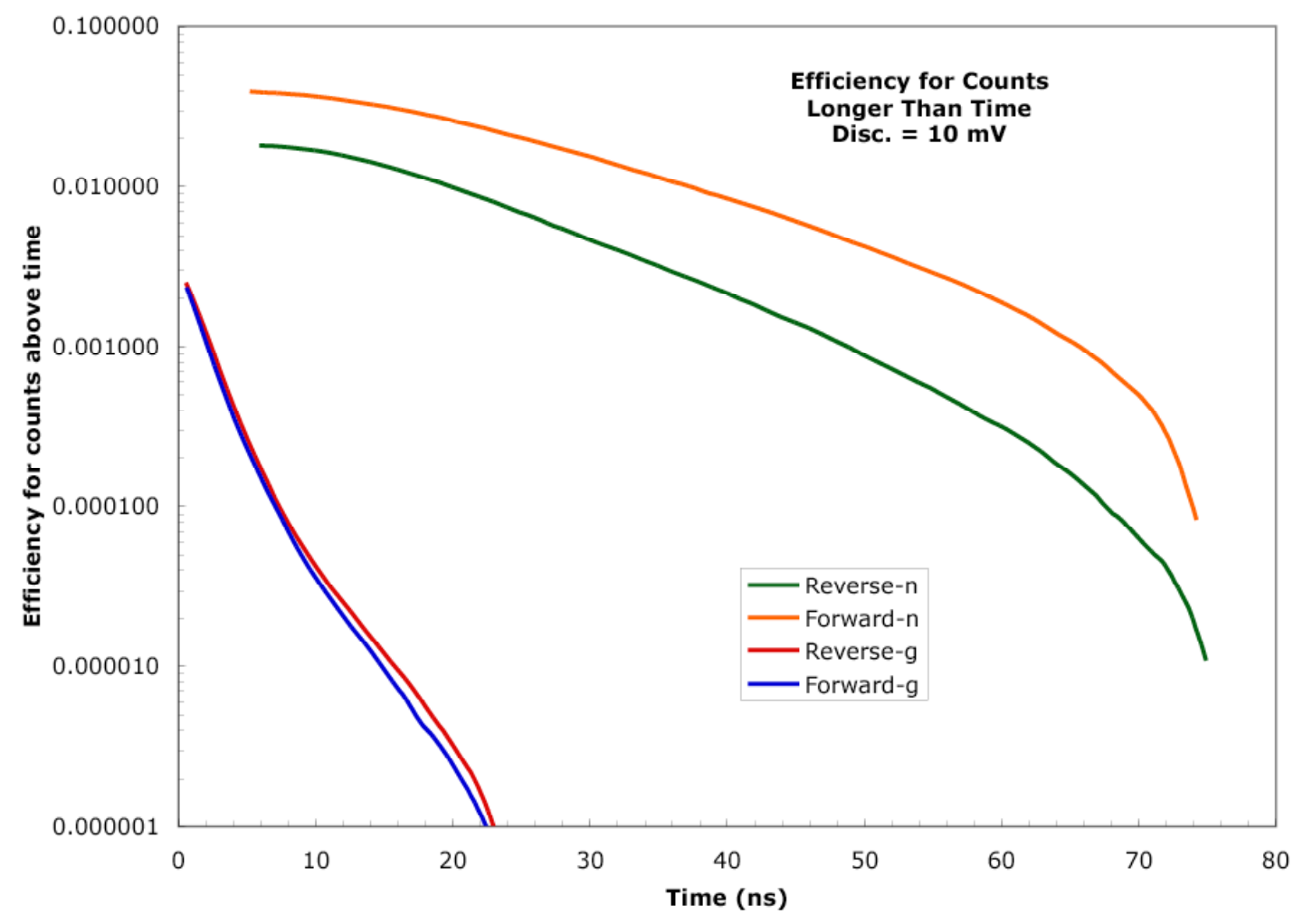

Figure S2. Intrinsic Fast Neutron $\left({ }^{252} \mathrm{Cf}\right)$ and Gamma $\left({ }^{137} \mathrm{Cs}\right)$ Detection Efficiency Measurements for the TOF Sensor at 10-mV PMT Discriminator Threshold, Plotted as a Function of the TOF Threshold Above Which the Sensor Response Is Integrated. The responses are divided into "Forward" and "Reverse" portions, corresponding to events traversing the sensor in the front-to-back direction and the back-to-front direction, respectively. The distinction is of greatest conceptual utility for the neutron source, in which reflection from the rear $\mathrm{Pb}$ shielding wall yields a substantial "Reverse" component to the sensor response.

In parallel with the experimental campaign, a Monte Carlo simulation of the TOF sensor was developed using the Geant 4 radiation transport toolkit. The main goals of the simulation effort at the outset of the project were to 1) guide interpretation of the experimental data and aid in planning additional measurements and 2) provide a rapid modeling tool for sensor design optimization, as verified against the benchmarking case provided by measurements with the existing ALPS II apparatus. The effectiveness of the modeling campaign with respect to the first purpose (especially in guiding interpretation of the "reflection effect" from the $\mathrm{Pb}$ wall evident in the negative-TOF portion of the neutron source data) was somewhat limited, given difficulties in rapidly defining and investigating a sensible set of timing variables in the simulation that could be meaningfully compared to data. Although the Geant 4 simulation framework supports optical photon tracking (in addition to a complete set of relevant physics governing interaction of ionizing radiation in materials), the computing time overhead associated with this tracking option was deemed prohibitive on the time-scale of this project. Instead, a substantially less central processing unit (CPU)-intensive "effective hit timing" approach, based on appropriate accounting of the tracking framework's time-stamp associated with each energy deposition event, or "hit," in a given scintillator slab, was adopted to facilitate rapid modeling of the TOF sensor. The extent to which this effort was successful can be judged on the basis of Figures S3 and S4, which display comparisons of, 
respectively, the simulated ${ }^{252} \mathrm{Cf}$ neutron TOF distribution to data, and the simulated intrinsic detection efficiency (for both ${ }^{137} \mathrm{Cs}$ gammas and ${ }^{252} \mathrm{Cf}$ neutrons) to data.

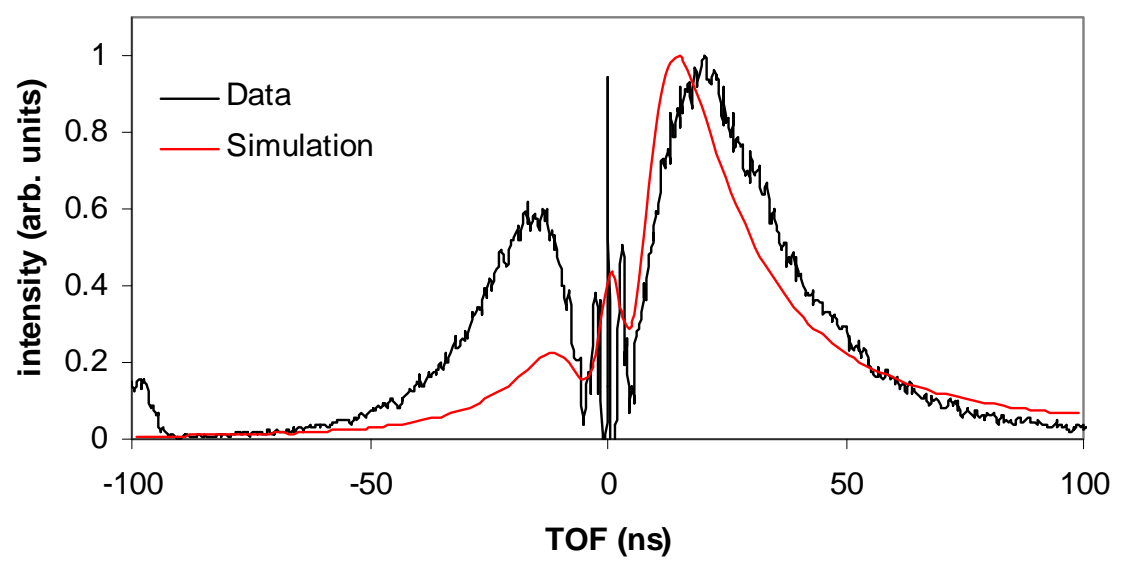

Figure S.3. Comparison of Simulated ${ }^{252} \mathrm{Cf}$ Neutron TOF Spectrum to Measured Spectrum. Both spectra are normalized to unity at the channel corresponding to the most probable TOF value. The data were recorded with 10-mV discriminator thresholds.

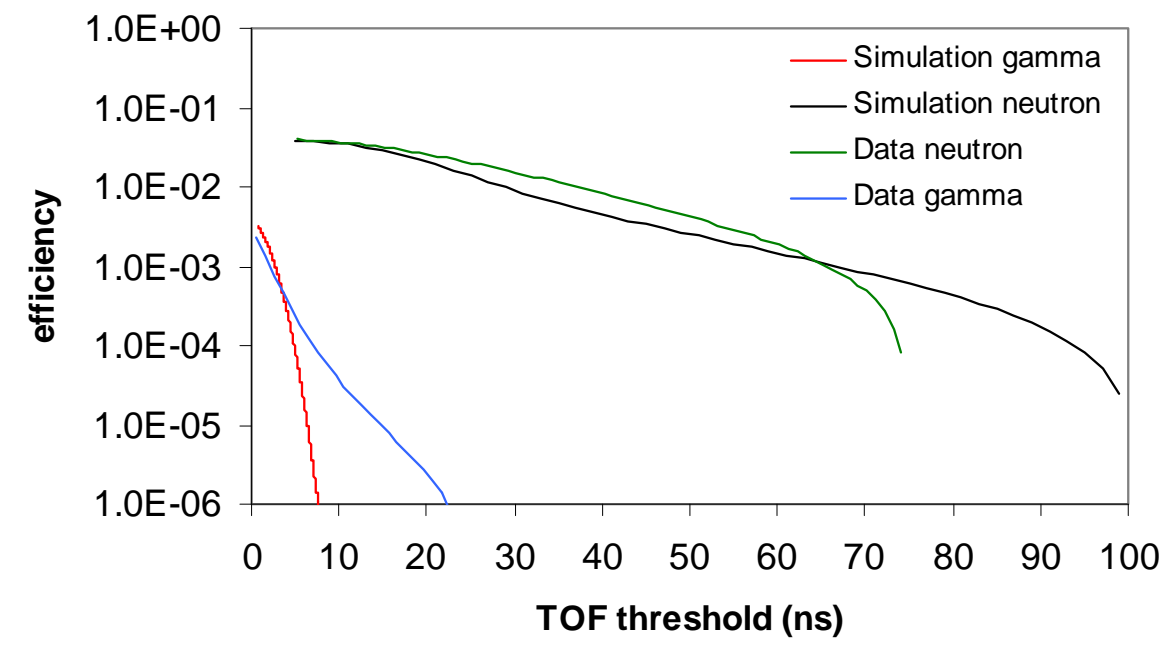

Figure S4. Intrinsic Detection Efficiency (detector response integrated above TOF threshold) as a Function of TOF Threshold for Mono-Energetic Gammas $\left({ }^{137} \mathrm{Cs} 662 \mathrm{keV}\right)$ and ${ }^{252} \mathrm{Cf}$ Neutrons. The data sets were recorded at 10-mV discriminator threshold; efficiencies correspond to the "Forward" portions of the TOF distributions. The simulation results correspond to energy deposition thresholds of $70 \mathrm{keV}$ (gamma) and $350 \mathrm{keV}$ (neutron), values that are consistent with one another given the differing light output of the secondary electrons and recoil protons that dominate the scintillator intearctions of gammas and neutrons, respectively.

The simulation code developed for this project fails to reproduce in detail the shapes of the gamma and neutron TOF distributions. The model departs from reality in the following major aspects:

- The most probable TOF value in the "Forward" lobe of the simulated neutron distribution is approximately $5 \mathrm{~ns}$ smaller than observed in the data. 
- The Pb-reflected component, or "Reverse" portion, of the neutron TOF distribution is substantially underpredicted in the simulation.

- The sensor's measured monoenergetic gamma TOF peak features non-Gaussian tails that are not predicted by the simulation.

However, despite these shortcomings, the model successfully describes the intrinsic detection efficiencies observed in the data for the ${ }^{137} \mathrm{Cs}$ gamma and ${ }^{252} \mathrm{Cf}$ neutron sources in terms of a consistent set of energy deposition thresholds for the two particle types. Because the only thresholds known a priori in the experimental apparatus are the discriminator levels for the PMT signal processing (expressed in essentially arbitrary signal voltages, rather than energies), the effective energy deposition threshold in the simulation constitutes a parameter that must be adjusted to achieve the best match between simulation and data. The best-match values of the simulation threshold are substantially different for the gamma and neutron source types ( 70 and $350 \mathrm{keV}$, respectively), but the two values can be reconciled by taking into account the differences in scintillation light yield for the relevant secondary particles (electrons and recoil protons for incident gammas and neutrons, respectively). Because the generation and tracking of scintillation light has not been explicitly modeled in the "effective hit timing" approach adopted for this simulation, the internal consistency of these two comparison benchmarks provides some confidence that the simulated efficiency results are, indeed, reasonable. To the extent that detector efficiency is reasonably well described, the code can be applied as a rapid modeling tool for design optimization in further n-TOF sensor work, with the essential caveat that the details of the TOF distribution in the region where the sensor's gamma response is changing rapidly (i.e., the non-Gaussian tail region extending roughly from 10 to $20 \mathrm{~ns}$ ) cannot be predicted accurately. The possibility of remedying this shortcoming by implementing more rigorous, albeit substantially more CPU-intensive, models of 1) scintillation photon propagation and 2) PMT pulse formation was not investigated in this project.

The expected performance of the TOF detection technology within the context of a realistic active neutron interrogation application was not thoroughly quantified in this project, either via measurements with a neutron generator or modeling simulations. However, the potential performance constraints imposed by high background singles rates (i.e., those background rates associated with pulses registered from individual scintillators or PMTs, as contrasted with time-coincident detection of particles interacting in both scintillators) are discussed in the final section of the report. It is shown there that an effective (point) source background gamma emission rate of $1 \mathrm{MHz}$ leads to a few 10s of $\mathrm{Hz}$ of accidental (i.e., uncorrelated) coincidence background, given a reasonable set of operational parameter assumptions, for deployment of a sensor comparable to the one investigated in this project. Further, because the accidental background rate scales as the square of the source emission rate, a factor of 10 increase in the effective source emission rate will raise this background event rate to over $1 \mathrm{kHz}$. In a similar vein, high PMT singles rates can lead to a "pre-emption" effect in which the time-interval information for one or more PMTs registered in the sensor's TDC can be shifted to smaller times. The estimated distortion of the TOF spectrum because of this effect, as measured by the number of events in the distribution displaced to incorrect times, is estimated to be a few percent at $100 \mathrm{kHz}$ single-PMT pulse rate.

The report concludes by comparing the intrinsic neutron detection efficiency of the present TOF sensor to a Monte Carlo N-Particle Transport Code (MCNP) simulation of a hypothetical, moderator-based ${ }^{3} \mathrm{He}$ tube sensor of comparable area. It is demonstrated there that the TOF method yields a fast neutron intrinsic detection efficiency ( $2.5 \%$ to $5 \%$, averaged over the ${ }^{252} \mathrm{Cf}$ emission spectrum, depending upon the TOF threshold) that compares favorably with the calculated efficiency of the moderator-based sensor 
(about $0.5 \%$ for the possibly under-moderated configuration assumed). Although the simple design adopted for the hypothetical ${ }^{3} \mathrm{He}$ sensor has not been subjected to performance optimization in any of its geometrical parameters, neither is the design grossly unrealistic (and, for that matter, the existing scintillator sensor's design has not been optimized for a TOF application, either). Thus, the comparison merely serves the purpose of highlighting the potential competitive position of the TOF method, especially in view of the cost benefits realizable from deploying plastic scintillator in a large-area sensor. 



\section{Acronyms}

ADC analog-to-digital converter

ALPS II

Advanced Large Area Plastic Scintillator (dual-sheet plastic scintillator sensor)

CPU

central processing unit

DFND

Direct Fast Neutron Detection

DHS

Department of Homeland Security

FWHM

full-width half-maximum

LLNL

Lawrence Livermore National Laboratory

MCNP

Monte Carlo N-Particle Transport Code

n-TOF

neutron time-of-flight

PNNL

Pacific Northwest National Laboratory

PMT

photomultiplier tube

QDC

charge-to-digital converter

SNM

special nuclear material

S\&T

Science and Technology

TDC

time-to-digital converter

TOF

time-of-flight 



\section{Contents}

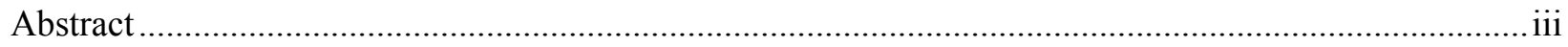

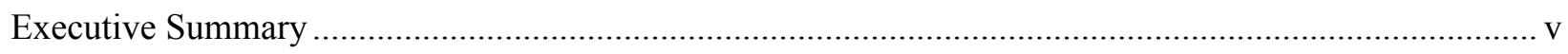

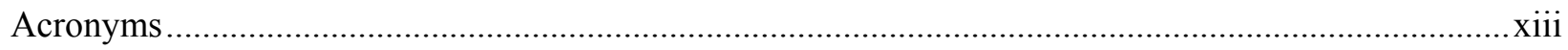

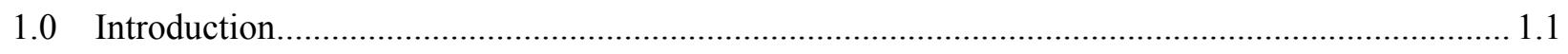

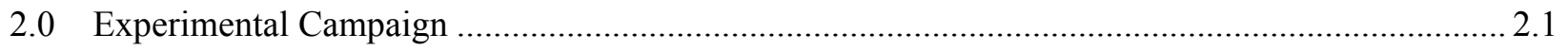

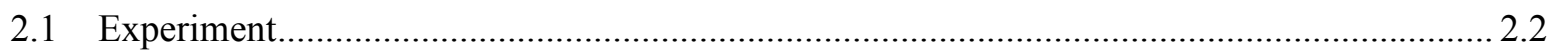

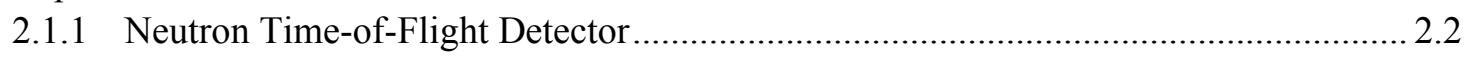

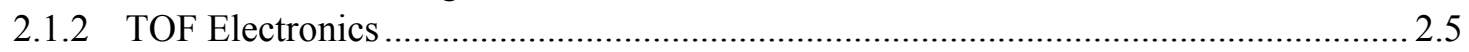

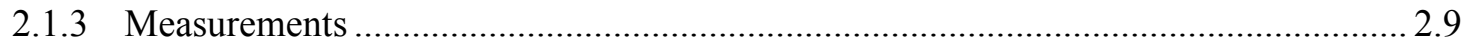

2.2 Results

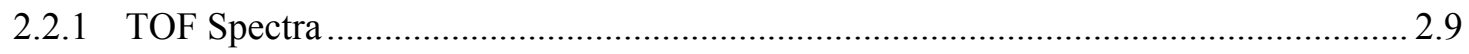

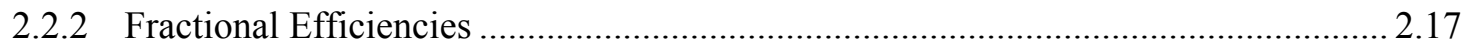

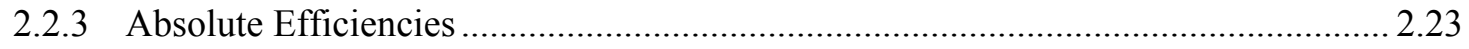

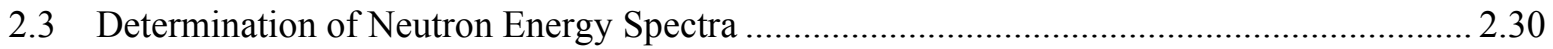

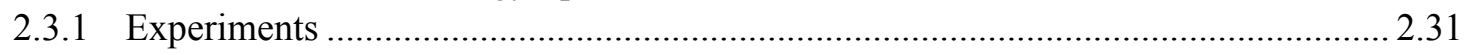

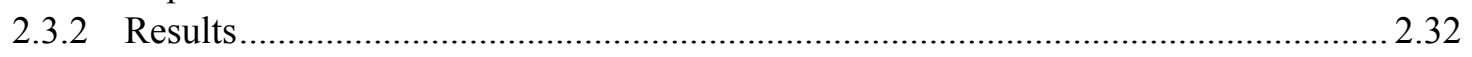

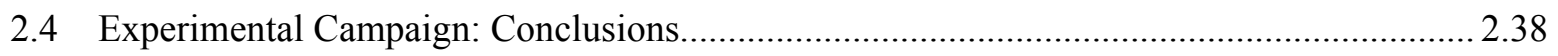

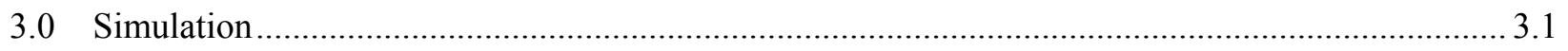

3.1 Simulation Results: Mono-Energetic Gamma Incident ................................................. 3.3

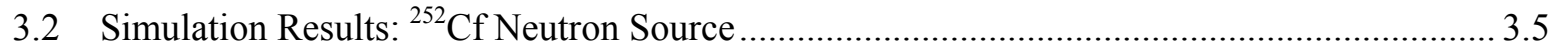

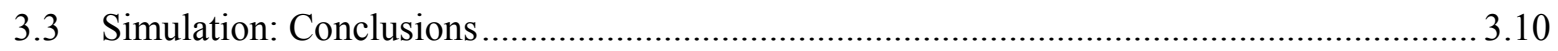

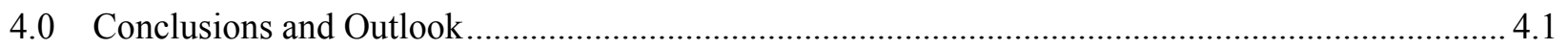

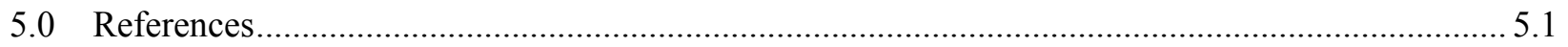




\section{Figures}

S1. Measured TOF Response of the $0.7 \mathrm{~m}^{2}$, Dual-Scintillator Sheet Sensor. vii

S2. Intrinsic Fast Neutron $\left({ }^{252} \mathrm{Cf}\right)$ and Gamma $\left({ }^{137} \mathrm{Cs}\right)$ Detection Efficiency Measurements for the TOF Sensor at 10-mV PMT Discriminator Threshold, Plotted as a Function of the TOF Threshold Above Which the Sensor Response Is Integrated viii

S.3. Comparison of Simulated ${ }^{252} \mathrm{Cf}$ Neutron TOF Spectrum to Measured Spectrum. ix

S4. Intrinsic Detection Efficiency (detector response integrated above TOF threshold) as a Function of TOF Threshold for Mono-Energetic Gammas $\left({ }^{137} \mathrm{Cs} 662 \mathrm{keV}\right)$ and ${ }^{252} \mathrm{Cf}$ Neutrons ix

1.1. $\mathrm{PuBe}$ (alpha, $\mathrm{n}$ ) TOF Spectrum Registered with a $30 \times 30 \mathrm{~cm}^{2}$ Dual-Sheet Plastic Scintillator Sensor Investigated in a Previous PNNL Project.

1.2. Comparison of (gamma-subtracted) TOF Spectra from Various Neutron Sources, Recorded with the $30 \times 30 \mathrm{~cm}^{2}$ Sensor of Stromswold et al.

2.1. Photograph of the Light-Tight Housing and Attached Electronics Cabinet for the Neutron TOF Sensor.

2.2. Detector Package for the n-TOF Sensor.

2.3. Cross-Sectional View of the n-TOF Sensor Showing the Arrangement of the Detector Package and Shielding.....

2.4. Conceptual Sensor Schematic Defining Timing Variables for Particle TOF and Interaction Position Reconstruction

2.5. Schematic of Data-Acquisition System

2.6. Time Distributions from Individual PMTs

2.7. TOF Spectra for the Background at Two Different Discriminator Levels

2.8. Comparison of ${ }^{137}$ Cs Gamma TOF Spectra for the Source Mounted at the Midpoint of the Steel Shield for Two Discriminator Levels

2.9. Comparison of ${ }^{137} \mathrm{Cs}$ Gamma TOF Spectra at a Discriminator Level of $100 \mathrm{mV}$ for the Standard Location and a Location 62.2-cm Farther Away

2.10. Comparison of ${ }^{137} \mathrm{Cs}$ Gamma TOF Spectra at a Discriminator Level of $10 \mathrm{mV}$ for the Standard Location and a Location 62.2-cm Farther Away

2.11. Comparison of Gamma TOF Spectra for Different Energy Gamma Sources at Two Discriminator Levels. 
2.12. Comparison of ${ }^{60} \mathrm{Co}$ TOF Spectra at Two Discriminator Levels .

2.13. Comparison of $\mathrm{Net}^{252} \mathrm{Cf}$ TOF Spectra After Normal Background Subtraction for the Source Mounted at the Midpoint of the Steel Shield for Two Discriminator Levels

2.14. Net ${ }^{252}$ Cf TOF Spectra After Normal Background Subtraction for Source Mounted Outside the Lead Shield at the Midpoint of the Backside of the Detector Housing.....

2.15. Comparison of net ${ }^{252} \mathrm{Cf}$ Neutron TOF Spectra After Subtraction of the ${ }^{252} \mathrm{Cf}$ Gamma Component for the Source Mounted at the Midpoint of the Steel Shield for Two Discriminator Levels.

2.16. Comparison of $\mathrm{Net}^{252} \mathrm{Cf}$ Neutron TOF Spectra for the Normal Source Location and a Location 62.2-cm Farther Away (discriminator level $=100 \mathrm{mV}$ ).

2.17. Comparison of $\mathrm{Net}^{252} \mathrm{Cf}$ Neutron TOF Spectra for the Normal Source Location and a Location 62.2-cm Farther Away (discriminator level $=10 \mathrm{mV}$ ).

2.18. Comparison of the ${ }^{137} \mathrm{Cs}$ Gamma TOF Spectrum and the ${ }^{252} \mathrm{Cf}$ Neutron TOF Spectrum at each of the four Discriminator Levels

2.19. Fractional Efficiency for ${ }^{137} \mathrm{Cs}$ as a Function of Time Threshold at each of the four Discriminator Levels

2.20. Comparison of Fractional Efficiency for ${ }^{137} \mathrm{Cs}$ in Reverse or Forward Distributions as a Function of Discriminator Levels

2.21. Fractional Efficiencies for ${ }^{137} \mathrm{Cs}$ at the Standard Source Distance and at a Distance 62.2-cm Farther Away

2.22. Relative Efficiency of Total Gamma Counts as a Function of Discriminator Level 2.20

2.23. Fractional Efficiencies for Reverse and Forward Neutron TOF Spectra for Four Discriminator Levels.

2.24. Comparison of Fractional Efficiency for ${ }^{252} \mathrm{Cf}$ in Reverse or Forward Distributions as a Function of Discriminator Levels

2.25. Fractional Efficiencies for ${ }^{252} \mathrm{Cf}$ at the Standard Source Distance and at a Distance 62.2-cm Farther Away

2.26. Relative Efficiency as a Function of Discriminator for Neutron TOF Spectra.

2.27. Neutron and Gamma Fractional Efficiencies

2.28. Absolute Efficiencies of the Gamma TOF Spectra as a Function of Time Threshold at each of the Four Discriminator Levels

2.29. Absolute Efficiencies for the Reverse and Forward Gamma TOF Spectra for all four Discriminator Levels. 2.25 xvii 
2.30. Absolute Efficiencies for Gamma TOF Spectra at the Standard Source Location and a Location 62.2-cm Farther Away

2.31. The absolute efficiency of total counts as a function of discriminator level for the Reverse,

Forward, and Total gamma TOF distributions.

2.32. Absolute Efficiency as a Function of Incident Gamma Energy for Sources at the Normal Location

2.33. Absolute Efficiency as a Function of Gamma Energy for Sources Located $62.2 \mathrm{~cm}$ from the Normal Position

2.34. Absolute Efficiencies for Neutrons as a Function of Time Threshold........................................ 2.28

2.35. Neutron Absolute Efficiencies for the Reverse and Forward Distributions................................ 2.29

2.36. Neutron Absolute Efficiencies as a Function of Time Threshold at the Standard Source Location and a Location 62.2-cm Farther away

2.37. Absolute Efficiency of Total Counts in Neutron TOF Spectra as a Function of Discriminator Level 2.30

2.38. Comparison of Absolute Gamma and Neutron Efficiencies as a Function of Time Threshold.....2.31

2.39. Pulse-Height Spectra for Three Gamma Sources Based on the Sum of the Pulse-Height Spectra of the Front and Back Scintillators

2.40. Pulse-Height Spectra for Two Discriminator Levels for the ${ }^{252} \mathrm{Cf}$ Source Based on the Sum of the Pulse-Height Spectra of the Front and Back Scintillators

2.41. TOF (horizontal axis) Versus Summed Pulse Height (vertical axis) for the ${ }^{60}$ Co Source with the TOF Discriminator at $20 \mathrm{mV}$.....

2.42. TOF (horizontal axis) versus Summed pulse height (vertical axis) for the ${ }^{252} \mathrm{Cf}$ source with the TOF discriminator at $100 \mathrm{mV}$.....

2.43. Total Pulse-Height Spectra for the ${ }^{252} \mathrm{Cf}$ Source for Different Processes Resulting from Gates set on the TOF Distribution

2.44. TOF (horizontal axis) Versus Pulse Height in the Back Scintillator (vertical axis) for the ${ }^{252} \mathrm{Cf}$ Source with the TOF Discriminators at $100 \mathrm{mV}$

2.45. TOF Spectrum Gated by a Narrow Region (channels 70-89) on the Back Scintillator Pulse-Height Axis

2.46. Location of Peaks on TOF Axis for TOF Spectra Gated by Narrow Bands on the Pulse-Height Axis for the Back Scintillator......

2.47. Location of Peaks on TOF Axis for TOF Spectra Gated by Narrow Bands on the Pulse-Height Axis for the Front Scintillator. 2.38 xviii 
3.1. Simulated ${ }^{137}$ Cs Gamma TOF Spectrum for the Dual-Sheet Sensor

3.2. Comparison of Simulated ${ }^{137}$ Cs TOF Spectrum (with 1.3-ns Gaussian smearing applied to both scintillator times before calculating TOF) to Data

3.3. Intrinsic Sensor Gamma Detection Efficiency as a Function of Threshold for the Data and the Simulation

3.4. ${ }^{252} \mathrm{Cf}$ Spontaneous Fission Neutron Spectrum Used as the Source Term for the n-TOF Sensor Response Simulation.

3.5. Simulated ${ }^{252} \mathrm{Cf}$ Neutron Spectrum Without (black curve) and with (red curve) Gaussian Smearing (1.3-ns standard deviation) Applied to the Time Signals from Both Scintillators

3.6. Comparison of Simulated ${ }^{252}$ Cf Neutron TOF Spectrum to Measured Spectrum.

3.7. Integrated Intensity Above TOF Threshold for the "Forward" Portion of the Sensor's ${ }^{252} \mathrm{Cf}$ Neutron TOF Distribution in Data (10-mV threshold) and Simulation.

3.8. Intrinsic Sensor Neutron Detection Efficiency as a Function of Threshold for the Data and the Simulation.

3.9. Intrinsic Detection Efficiency (detector response integrated above TOF threshold) as a Function of TOF Threshold for Gammas $\left({ }^{137} \mathrm{Cs} 662 \mathrm{keV}\right)$ and Neutrons $\left({ }^{252} \mathrm{Cf}\right)$

4.1. Calculated Gamma-Induced Accidental Trigger Rate as a Function of Point-Source Emission Rate for a TOF Sensor of the Same Dimensions as that Used in this Project.

4.2. Cross-Sectional Schematic of Moderated ${ }^{3} \mathrm{He}$ Neutron Detector Modeled in MCNP

\section{Table}

2.1. Weighted Average Channel Numbers for Pulse-Height Spectra Derived from Two-Dimensional Plots of Pulse-Height Spectra Versus TOF. 


\subsection{Introduction}

Neutron detection is a critical enabling technology for interdiction of covertly-transported special nuclear material (SNM), safeguards and security monitoring at nuclear reactor facilities, and characterization of uranium or transuranic materials (Reilly 1991). Measuring neutron flux can provide valuable information about the presence, quantity, and nature (distribution, chemical form, isotopic distribution, etc.) of uranium or transuranic elements in cargo, waste, or other matrix. Passive technologies rely upon neutron emission arising from spontaneous fission or from the (alpha, $n$ ) class of nuclear reactions with light elements in the matrix. Active and "near-passive" technologies use an external neutron source and rely upon neutron emission resulting from induced fission. Both approaches rely on neutron detection.

Conventional approaches to neutron detection require slowing incident neutrons with a moderator and recording their presence via any of a number of useful nuclear reactions (e.g., ${ }^{3} \mathrm{He}(\mathrm{n}, \mathrm{p})$ ). The moderation process, which is necessary to achieve reasonable detection efficiency, precludes any opportunity to recover much of the information carried by a source neutron. Timing information, for example, could in principle be exploited to measure coincident events, to reject pulsed interrogation neutrons, or even to locate neutron sources through measurement of "time-of-flight" (TOF). The moderation process, however, is a random process that can require anything between 1 and $100 \mu \mathrm{s}$, depending on the composition and arrangement of moderating materials. Moderation times between 10 and $30 \mu$ s are typical. For many applications, therefore, conventional neutron detection approaches preclude timing accuracies better than roughly $30 \mu \mathrm{s}$. Moderation also destroys information about the energy of individual neutrons because all incident neutrons are moderated to thermal energies before detection. It is still possible to extract neutron spectral information from a moderator-based detection method (e.g., a Bonners sphere set) by exploiting statistical characterizations of the dependence of neutron moderation and transport upon incident energy (Toyokawa 1996; Toyokawa 1997; Yamaguchi 1999). Such "statistical spectroscopy" devices, however, require measuring relatively large populations of neutrons to reliably reconstruct the incident energy spectrum. In addition, the relationship between the incident neutron spectrum and the device response depends in general on the detector's physical surroundings because of the moderation of neutrons in materials close to the detector. Thus, these devices are of limited utility for detection scenarios requiring rapid measurements and/or flexibility in deployment surroundings. Finally, moderation also obscures or destroys information about the incident direction of source neutrons because the process of moderation typically involves numerous individual scattering events that completely randomize a neutron's direction.

In contrast to detectors relying on the moderation of neutrons to thermal velocities as a prerequisite for achieving reasonably efficient detection, "fast neutron detectors" are here defined as those that have the capability to detect fast neutrons (energies in the range $100 \mathrm{keV}$ to $20 \mathrm{MeV}$ ) efficiently without the need for moderation (Reeder et al. 1999; Hansen et al. 2000). A fast neutron detector exploits a "direct" neutron interaction with an appropriate detection medium that 1) occurs with sufficiently high cross section at fast neutron energies that moderation is rendered unnecessary and 2) yields a signature amenable to convenient extraction from the medium. Such a detector offers the potential for significant enhancement in the quality and precision of information (e.g., time, location, and energy of emission of the event) registered from incident neutrons on an individual-particle basis. It is fair to characterize neutron detection applications in national security as dominated by moderator-based methods (Peurrung 2000), in no small part because ${ }^{3} \mathrm{He}$ tubes offer the user community a convenient, well- 
understood, and commercially available enabling technology for implementing this approach. However, one notes that a technology capable of directly detecting fast neutrons without moderation has access to much more information about the neutron source and can thus enable a variety of advanced technologies. A partial list of challenges that can be effectively addressed by direct fast neutron detection (DFND) is given below:

- Improve active technology: Active interrogation technology could conceivably be significantly improved were it possible to effectively discriminate between those neutrons that arise from the interrogation source and those that arise within the medium undergoing characterization. Conventional moderate-and-capture neutron detection methods are poor at enabling such discrimination. DFND, on the other hand, would enable discrimination on the basis of timing, directionality, or energy. An interesting example of such an approach is found in the fusion diagnostic literature (Fisher et al. 1997).

- Image neutron sources: The capability to directly image a neutron flux can enable more sensitive nuclear materials detection in a manner parallel to that provided by gamma ray imagers.

Analogous gamma-ray detection technologies have enabled gamma-ray imaging using methods such as Compton or coded aperture imaging. (See, for example, Woodring 1999 or Anderson et al. 2005.) Maximizing the usefulness of this approach for neutron detection will require enabling technologies capable of registering, at a minimum, the location of a fast neutron's first interaction. This interaction-position information is necessarily smeared in a moderator-based detector because of the finite-length "random walk" suffered by a neutron as it thermalizes in the moderator. A DFND method offers the potential for substantially improving the precision of the neutron interaction position measurement because this precision is limited only by the position resolution of the signature extracted from the detection medium (e.g., bubble nucleation position in a recoil-nucleation detector, or scintillation light emission position in a proton-recoil detector), and not by the spatial dispersion in the neutron's stochastic path through the moderator. DFND technologies in general are thus expected to substantially enhance neutron imaging capability.

- Improve coincident-detection approaches to neutron assay: Accurate quantification of SNM content, especially under adverse circumstances, is hindered by the lack of neutron detectors capable of recording accurate timing information. Timing information can be used in a number of ways with the result of greatly improved characterization. One obvious approach is to record coincident pairs of neutrons arising from spontaneous or induced fission events. (No (alpha, n) events can be detected in this way, which is frequently useful.) However, a detector with poor timing must cope with the serious problem of "accidental" coincidence signal. DFND methods typically enable timing that is at least an order of magnitude better than that provided by conventional detectors that must moderate neutrons before detection.

- Facilitate neutron detection in the presence of strong gamma-ray fluxes: Gamma interactions in the walls of ${ }^{3} \mathrm{He}$ and $\mathrm{BF}_{3}$ proportional tubes generate secondary electrons that traverse the tube gas. These secondary electron tracks can produce ionization in the gas and, if the gamma flux is sufficiently intense, will yield pulses of amplitude comparable to neutron-induced pulses. Thus, moderator-based neutron detectors typically face severe challenges when required to operate under high gamma-flux conditions, and the detector's capability to distinguish neutrons from gammas may be lost. Even when operation in a strong gamma field is possible, the specialized pulse-processing techniques required to permit amplitude discrimination against gammas 
generally compromise neutron detection efficiency. At least one type of fast neutron detector, namely the recoil-induced bubble nucleation detector, is inherently gamma-blind and ought thus to be able to operate in arbitrarily high gamma-ray fluxes (Schulze et al. 1992).

As these challenging pathways to detector performance improvement suggest, fast neutron detection evidently offers substantial promise for extracting maximum information content from the neutrons emitted by sources of interest in both passive and active detection applications. A range of methods have been the subject of research and have shown the ability to detect fast neutrons in a way that is both efficient and insensitive to gamma-ray radiation. Pacific Northwest National Laboratory (PNNL), in particular, has been engaged in such research since roughly 1994. Highlights of the fast neutron detection program at PNNL include the following:

- Fast neutron detection via bubble chambers: A PNNL exploratory research project in FY 2003 clearly demonstrated the feasibility of detecting fast neutrons with a pressure-cycled bubble chamber (Jordan et al. 2005). Bubble formation occurs due to the elastic scattering of neutrons from target nuclei in the superheated working fluid, producing highly-ionized, relatively slow-moving recoil ions. These recoil ions can deposit sufficient energy in a small enough space within the fluid to induce bubble nucleation. The energy deposition required for nucleation, typically several hundred $\mathrm{eV}$ to a few $\mathrm{keV}$, cannot be supplied by a thermal neutron, and thus the technology is inherently blind to thermals. Discrimination against gammas is achieved by selecting suitable chamber operating conditions such that the secondary electrons produced by gamma rays deposit energy too diffusely to nucleate bubbles. The bubble chamber is a pressure-cycled device that promises excellent detection efficiency (of order several 10's of percent) over a limited portion of the chamber's pressure cycle. Maximum cycling frequencies are anticipated to be on the order of $1 \mathrm{~Hz}$, with a sensitive time window of duration 10 to $50 \mathrm{~ms}$ per cycle. The bubble chamber thus offers a promising enabling technology for neutron generator-based active interrogation methods, since the chamber's neutronsensitive time window can be phase-locked to the neutron generator's operating cycle. The chamber can thus be made completely insensitive to the generator neutron pulse, and can map out the time structure of the return flux of neutrons from an interrogated sample or search area. In addition, the bubble location marks the position of a single neutron interaction, and can be determined by optical or acoustic means to a position resolution on the order of millimeters. The bubble chamber thus offers significant potential for neutron imaging applications. And finally, the dependence of the chamber's fast neutron energy threshold on the working fluid's superheat (directly related to the pressure drop experienced by the chamber fluid upon expansion) permits mapping of the energy distribution of the incident neutron flux, as first proposed in (Fisher et al. 1997). The bubble chamber thus offers a promising technological path forward for achieving fast neutron spectroscopy.

- Direct fast neutron detection by Time-of-Flight: Multiple-sheet plastic scintillator sensors can be used to exploit the TOF method for measuring the energy of individual neutrons without moderation. The method relies on the successive elastic scattering of a neutron in two sheets of plastic scintillator, separated by a distance on the order of a few 10's of cm. A PNNL project in the late 1990's (Stromswold et al. 1999) demonstrated that a fast neutron sensor consisting of two $30 \times 30 \mathrm{~cm}^{2}$ slabs of 2"-thick plastic scintillator could detect fast neutrons with an efficiency on the order of $1.5 \%$, and with clear spectral sensitivity to the incident neutron source type (e.g. AmBe, ${ }^{252} \mathrm{Cf}, \mathrm{PuBe}$, and $\mathrm{PuO}_{2}$ ). Refinements of the baseline technique explored in that project could include segmentation of the scintillator panels to permit enhanced TOF resolution. 
An important goal of the Methods and Instruments for Fast Neutron Detection project, a sub-task of PNNL's Department of Homeland Security (DHS) Science and Technology (S\&T) Rad/Nuc Countermeasures: Advanced Sensors project, has been to develop and test fast neutron detection technology suitable for passive and near-passive measurements of SNM in support of Lawrence Livermore National Laboratory's (LLNL's) Advanced Concept project under the Active Detection program. In order to exploit existing equipment and experience at PNNL, and because substantial engineering challenges face development of the pressure-cycled bubble chamber approach before this technology reaches a stage of maturity appropriate for inclusion in LLNL's sensor system, the Methods and Instruments project has focused on quantifying the fast neutron detection performance of a large-area $\left(\sim 0.7 \mathrm{~m}^{2}\right)$, monolithic, two-sheet plastic scintillator neutron time-of-flight (n-TOF) sensor. A plastic scintillator n-TOF sensor detects fast neutrons via successive elastic scatterings in two spatially separated scintillators. Gamma discrimination is achieved by segregating a portion of the TOF distribution corresponding to the fastest particles traversing the sensor. By placing a cut on this "gamma flash" portion of the distribution, implemented either in the sensor's hardware trigger or in software, the system's responses to gamma and neutron fields can be distinguished.

A large-area scintillator TOF sensor offers several attractive benefits in support of a passive or "near passive" neutron detection program. First, monolithic plastic scintillator slabs scale readily and costeffectively to large areas and can thus provide broad solid-angle coverage; this is an important factor in achieving reasonably high absolute detection efficiency. In addition, the timing resolution achievable with a plastic scintillator-based system should permit substantially more precise time windowing of return neutron flux (in a "near passive" interrogation system) than would otherwise be possible with moderated detectors such as ${ }^{3} \mathrm{He}$ tubes. High-resolution time windowing can provide a powerful tool for background suppression in an active system in which the sensor response can be phase-locked to the generator of the interrogating radiation. With these performance goals in mind, the primary technical challenges addressed in the current project were to understand and quantify the capabilities, including fast neutron detection efficiency and effectiveness of neutron/gamma discrimination, of an n-TOF sensor in the limit of large scintillator area. This is a size regime in which the neutral particle's flight path between the two scintillators is not tightly constrained.

The current project leverages prior PNNL experience with a relatively small area $(30 \mathrm{~cm} \times 30 \mathrm{~cm}=$ $0.09 \mathrm{~m}^{2}$ ) n-TOF scintillator sensor investigated in the course of an NA-22 funded project in the late 1990s (Stromswold et al. 1999). The primary goal of that experimental work, the so-called DFND project, was to achieve neutron detection with robust discrimination of gammas from neutrons in a single sheet of plastic scintillator by exploiting differences in the time dispersions of the interaction mechanisms (Compton scattering and/or photoelectric absorption for gammas, elastic proton recoil for neutrons) that deposit energy in the scintillator for the two particle types. This aspect of the DFND work did not yield results sufficiently promising to warrant further pursuit of the single-scintillator neutron detection method at that time. However, in a second phase of the project, the neutron detection capabilities of a $30 \times 30$ $\mathrm{cm}^{2}$ dual-sheet plastic scintillator TOF sensor (with adjustable sheet separation distance) were investigated, and the results of this study were promising:

- A clear distinction between the gamma "flash" peak in the TOF distribution and the neutron portion of the distribution was observed, as displayed in Figure 1.1 for a $30-\mathrm{cm}$ sheet separation. Increasing the separation between the scintillator sheets increases the "lever arm" for the TOF measurement, and thus improves the separation between the two portions of the spectrum, at the expense of reducing the sensor's absolute detection efficiency (because of the decreased solid 
angle for registering an interaction in the second scintillator sheet after an initial scattering event in the first sheet).

- An intrinsic detection efficiency (i.e., absolute efficiency expressed as the ratio of number of particles detected to the number of particles incident on the sensor) of approximately 1.0 to $1.5 \%$ was achieved for energetic (alpha, n) neutrons from a PuBe source.

- Spectral differentiation of several fast neutron source types $\left(\mathrm{PuBe}, \mathrm{PuO}_{2}\right.$, and $\left.{ }^{252} \mathrm{Cf}\right)$ was possible on the basis of the shape of the TOF distribution; see Figure 1.2.

- The system's pulse-processing electronics permitted measuring a maximum neutron TOF of approximately $50 \mathrm{~ns}$, or 0.05 microseconds, a timing interval sufficiently broad to encompass essentially the entire PuBe energetic neutron spectrum of interest. This coincidence resolving time represents a marked improvement over the performance of moderate-and-capture systems, for which the relevant time scale is typically microseconds or more.

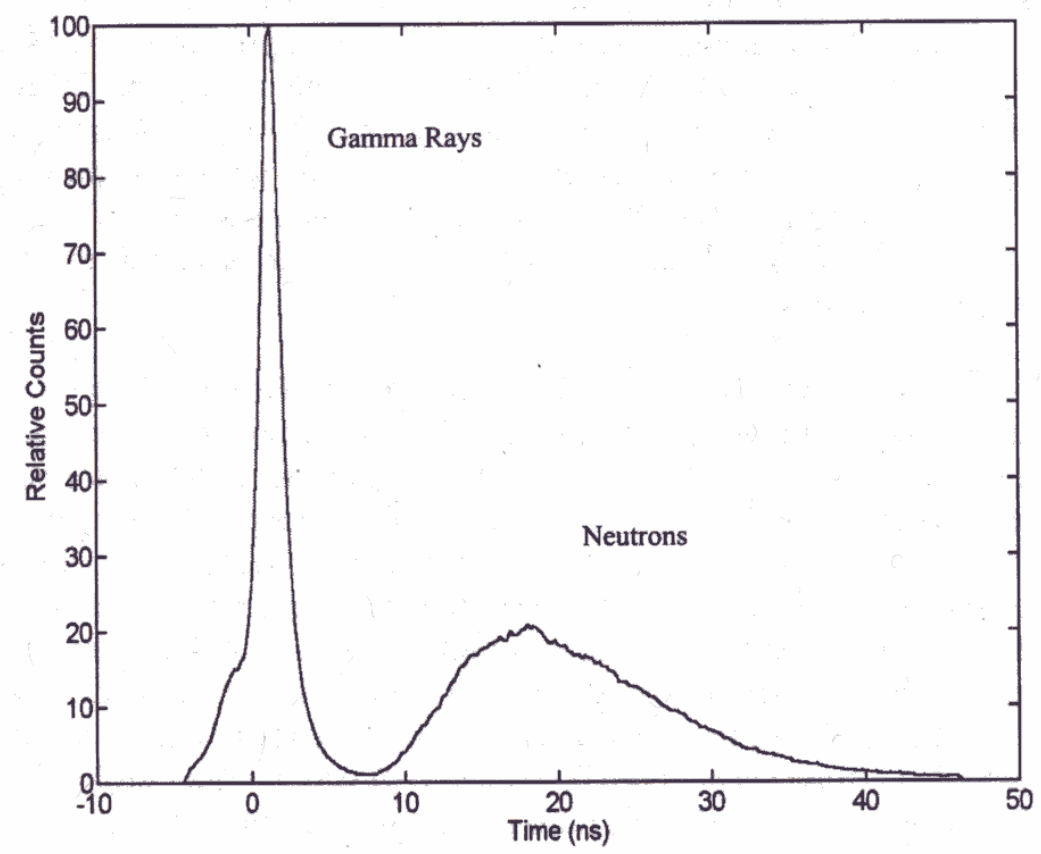

Figure 1.1. PuBe (alpha, n) TOF Spectrum Registered with a $30 \times 30 \mathrm{~cm}^{2}$ Dual-Sheet Plastic Scintillator Sensor Investigated in a Previous PNNL Project (Stromswold et al. 1999). The two scintillator sheets are separated by $30 \mathrm{~cm}$ (center-to-center). 


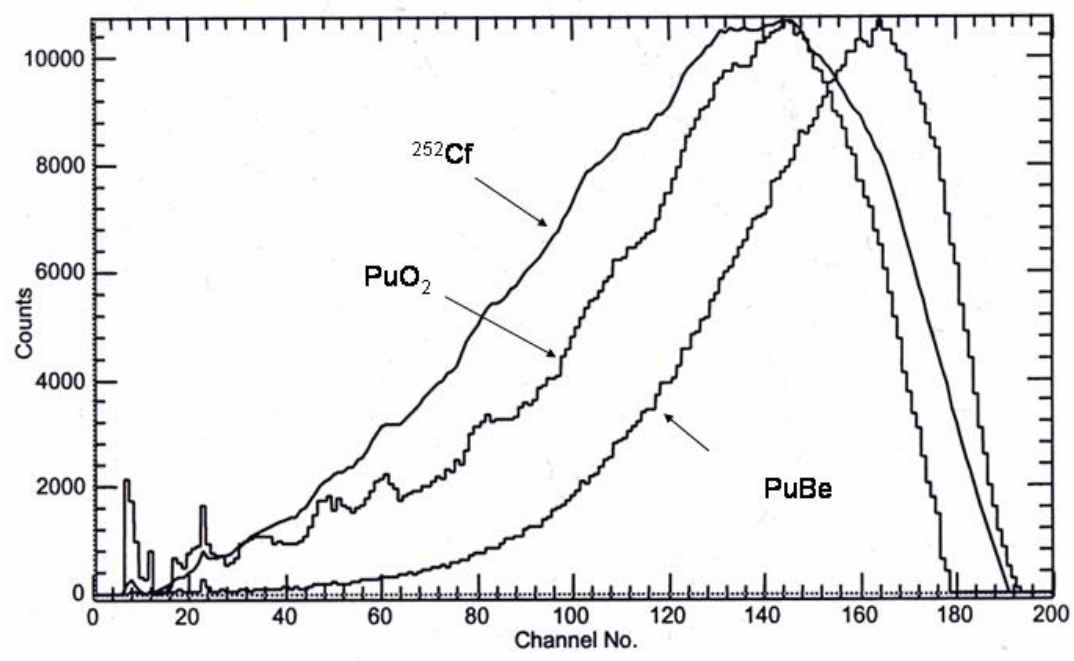

Figure 1.2. Comparison of (gamma-subtracted) TOF Spectra from Various Neutron Sources, Recorded with the $30 \times 30 \mathrm{~cm}^{2}$ Sensor of Stromswold et al. (1999)

The dual-sheet scintillator sensor investigated in the current Methods and Instruments project represents nearly an order of magnitude increase in sensor area $\left(0.73 \mathrm{~m}^{2}\right)$ over that of the earlier DFND project. The n-TOF sensor consists of two aluminized-mylar wrapped sheets of $127 \mathrm{~cm} \times 57.2 \mathrm{~cm} \times 5.0 \mathrm{~cm}(50 \mathrm{in} . \times$ 22.5 in. $\times 2$ in.) BC-408 scintillator, separated by a center-to-center distance of $17.8 \mathrm{~cm}(7 \mathrm{in}$.). The shorter ends of each scintillator sheet are outfitted with three $12.7 \mathrm{~cm}$ (5 in.) diameter Hamamatsu photomultiplier tubes (PMTs) apiece, for a total of 12 PMTs. Experimental work in this project focused on recording timing information from only four of these PMTs, one at the center of each scintillator end.

Extracting optimum performance from a large-area n-TOF sensor requires correcting the nominal (i.e., normal-incidence) flight path for the actual interaction positions within each scintillator because flight paths for obliquely-scattered particle tracks may be an order of magnitude larger than the scintillator separation. To the extent that the actual flight path can be determined, the particle velocity can be calculated from the ratio of the flight path to the TOF. The goal of calculating the particle velocity is to sharpen the distinction between the distributions corresponding to the gamma "flash" and the (slower) neutrons. It was anticipated at the outset of this project that flight-path corrections of sufficient accuracy to permit a significant improvement of gamma/neutron discrimination could be achieved with a minimum of position-sensitive detection apparatus via relative timing-based determinations of interaction position within each scintillator. A crude measure of the "long-axis" interaction position can be obtained in a double-ended scintillator sensor by determining the difference of signal arrival times from the PMTs at the two ends. In practice, this approach did not bear fruit in the apparatus used for this project because of ambiguities in the lateral (i.e., "short-axis") interaction position within each scintillator, a coordinate that is not well-measured by a dual-ended PMT configuration. 


\subsection{Experimental Campaign}

The primary mechanism for detecting gammas by TOF in plastic scintillators is Compton scattering. A gamma typically interacts in one scintillator by scattering off an electron. Part of the energy of the gamma is given to the electron as recoil energy, and the rest of the energy is in the scattered gamma. The recoiling electron produces a pulse in the first scintillator, and the lower-energy scattered gamma is detected via a second Compton scattering or photoelectron capture event in the second scintillator. The measured TOF is for the flight time of the scattered gamma from one detector to the other. The flight times expected for these experiments are $<5 \mathrm{~ns}$ because the gamma is traveling at the speed of light in air $(30 \mathrm{~cm} / \mathrm{ns})$.

Detecting neutrons by TOF depends on a different mechanism. Typically, a neutron elastically scatters off a proton in the first scintillator, giving part of its energy to the recoil proton, and then continues to the second scintillator where it again elastically scatters off a proton. The pulse observed in the first scintillator is caused by the recoil proton from the first scattering, and the pulse in the second scintillator is caused by the recoil proton from the second scattering. The measured TOF is the flight time for the neutron after its first scattering event. The neutron is a massive particle whose velocity can be expressed by the non-relativistic equation:

$$
v_{n}=\sqrt{E_{n} / 0.5165}
$$

where $\mathrm{v}_{\mathrm{n}}$ is the velocity of the neutron in $\mathrm{cm} / \mathrm{ns}$, and $\mathrm{E}_{\mathrm{n}}$ is the neutron energy in $\mathrm{MeV}$.

For the neutron energies of interest here $(<20 \mathrm{MeV})$, the velocities are $<6.2 \mathrm{~cm} / \mathrm{ns}$. Expected flight times are in the range of 2 to $100 \mathrm{~ns}$.

The highest energy neutrons have short flight times that overlap with the gamma flight times. Fortunately, the number of high-energy neutrons in this time region is a small fraction of the total neutrons of interest here. In practice, a time threshold will be set so that only events with a time greater than the threshold will be counted as neutrons. One of the goals of this work is to determine the probability of a gamma event exceeding various thresholds. There is a trade-off between maximizing the neutron efficiency by lowering the time threshold and minimizing the gamma contamination by raising the time threshold.

TOF measurements were made with a calibrated ${ }^{252} \mathrm{Cf}$ neutron source and a calibrated ${ }^{137} \mathrm{Cs}$ gamma source to measure the relative and absolute efficiencies of detecting each type of radiation. Of particular interest is the efficiency above a given TOF threshold so that the contamination of the neutron TOF spectrum by gammas could be determined. In addition, a few measurements were made with calibrated ${ }^{54} \mathrm{Mn}$ and ${ }^{60} \mathrm{Co}$ gamma sources to see whether the initial gamma energy influenced the results. We also report a few measurements of pulse-height spectra obtained by a multi-channel charge-to-digital converter performed simultaneously with TOF measurements. 


\subsection{Experiment}

\subsubsection{Neutron Time-of-Flight Detector}

The initial experiments were done with an existing, NA-22 funded assembly known as the Advanced Large-Area Plastic Scintillator (ALPS II) detector (Reeder et al. 2003). The detector, which was designed, fabricated, and previously studied in the context of gamma detection for portal monitor applications, consists of two slabs of BC-408 plastic scintillator of dimensions $127 \times 57.15 \times 5.08 \mathrm{~cm}^{3}$. A sheet of $0.32-\mathrm{cm}$ thick lead is between the two slabs to reduce scattering of low-energy gammas from one scintillator to the other. The slabs are separated by a distance of $13 \mathrm{~cm}$. The slabs are each wrapped in aluminized Mylar on all faces except the $57.15 \times 5.08 \mathrm{~cm}^{2}$ ends. Each end of each scintillator is viewed by three Hamamatsu R1250 5-in. (12.7-cm) diameter PMTs. The PMTs cover about $67 \%$ of the exposed area. However, the initial TOF experiments were done using only the center PMT of each end to simplify the data acquisition.

The scintillators are shielded by 5.08-cm-thick lead on the bottom, sides, and back and are encased in a light-tight steel box. A light-tight plastic door allows neutrons and gammas to enter the front face of the assembly. To minimize the gamma sensitivity of this detector, an external shield of 2.54-cm-thick steel covered the front face. Figures 2.1 through 2.3 display, respectively, a photograph of the sensor in its light-tight housing, CAD diagrams of the detector package, and a cross-sectional view of the sensor and shielding materials. 


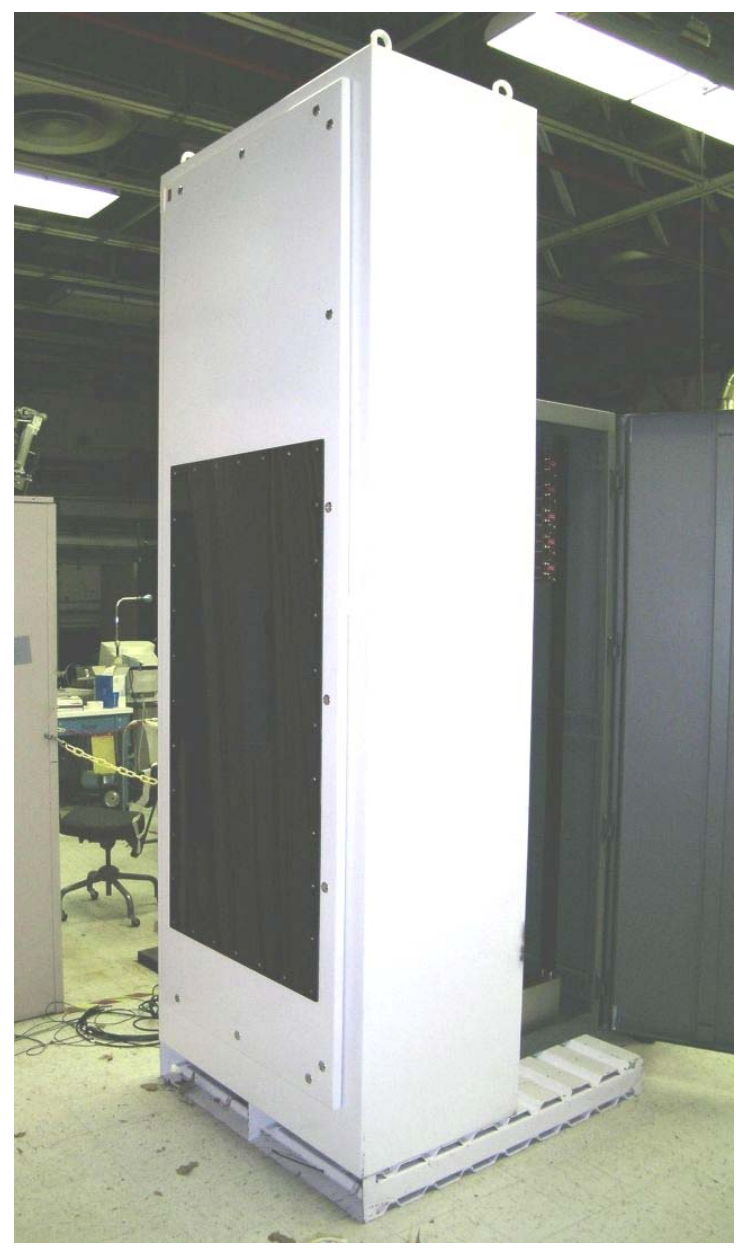

Figure 2.1. Photograph of the Light-Tight Housing and Attached Electronics Cabinet for the Neutron TOF Sensor. A 2.54-cm-thick steel plate, mounted at the front (left) entrance of the sensor throughout the experiments described in this report, is not pictured. 


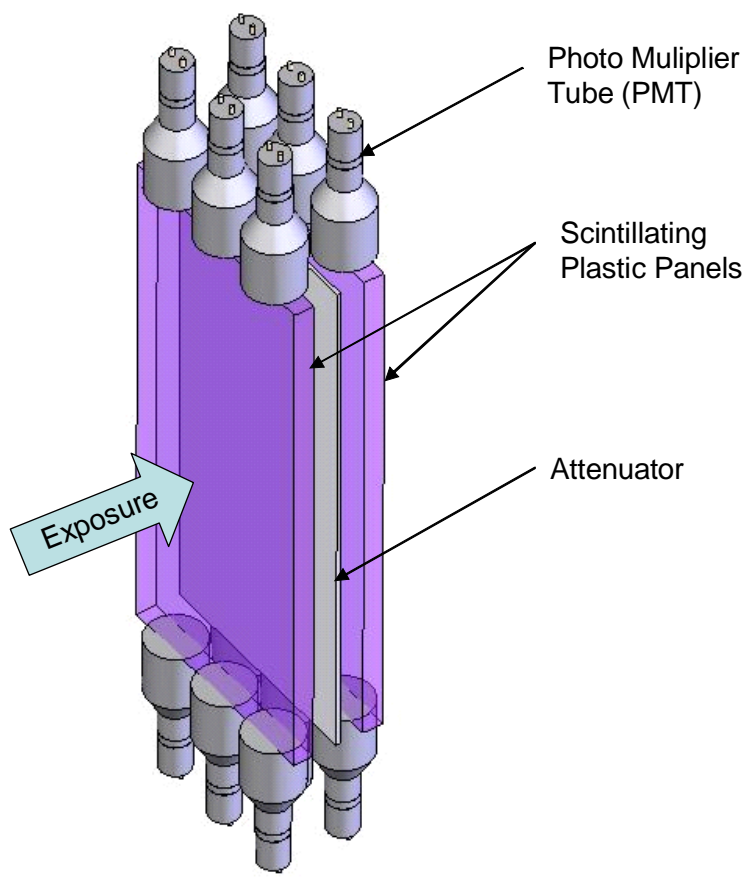

Figure 2.2. Detector Package for the n-TOF Sensor. Note that only the center PMTs on each scintillator sheet end are used to define the sensor's event trigger and TOF variable.

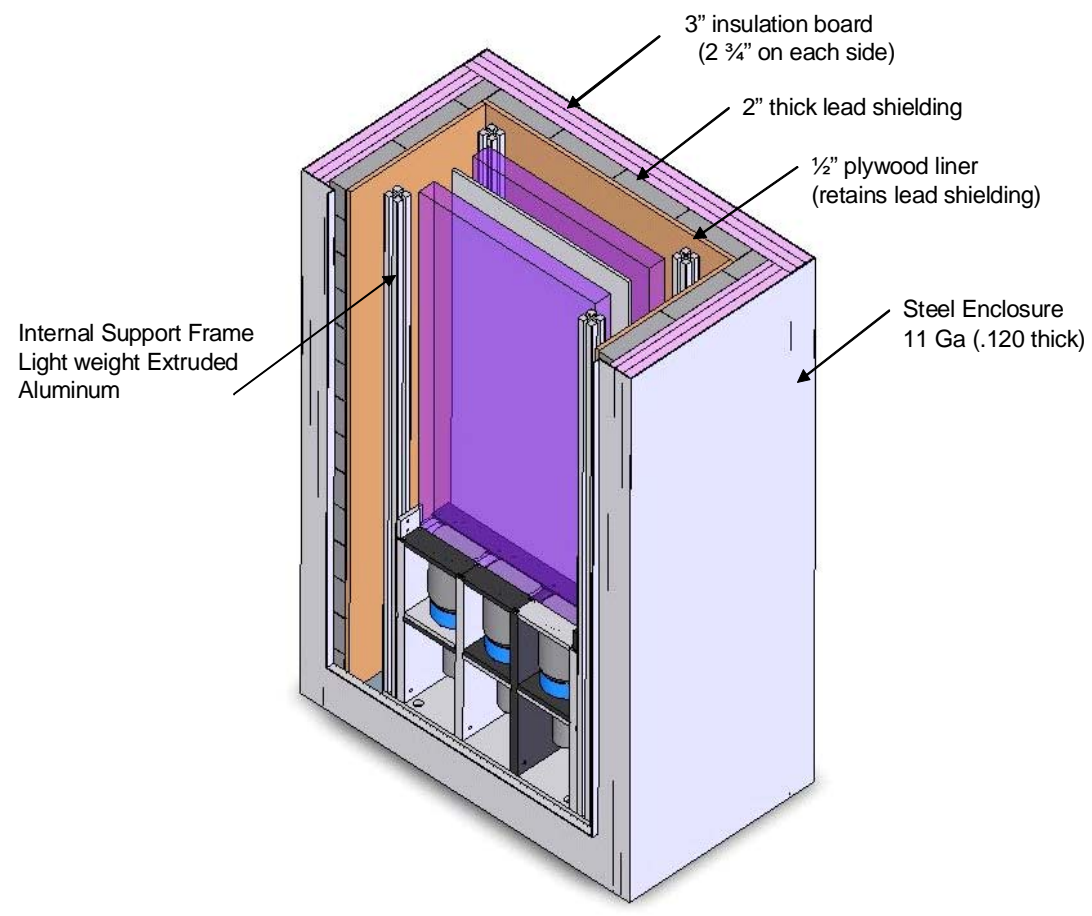

Figure 2.3. Cross-Sectional View of the n-TOF Sensor Showing the Arrangement of the Detector Package and Shielding 


\subsubsection{TOF Electronics}

Figure 2.4 displays a conceptual schematic of the sensor timing variables required to reconstruct the particle TOF (and, in principle, the interaction position of the particle within each scintillator). The "particle trajectory" depicted in the figure is idealized in the sense that a scattering event within the first scintillator will, in general, lead to a finite scattering angle and thus a "kink" in the trajectory at the first scattering position. The $\mathrm{X}$-axis in this figure lies along the long-axis of the scintillators; note that the figure fails to represent the dispersion of the interaction position along the transverse direction, or Y-axis (i.e., perpendicular to the plane of the figure). The incident particle scatters at position $X_{1}$ in the first scintillator sheet, as measured from one end of the sheet. Scintillation light propagates from this interaction position to the two ends of the scintillator with velocity $\mathrm{c} / \mathrm{n}$, where $\mathrm{c}$ is the speed of light in vacuum, and $\mathrm{n}$ is the index of refraction of the scintillator. Analog electronic pulses generated by the PMTs (not depicted in the diagram) traverse signal cables leading to discriminators, and the signal from the "left" end of the first scintillator provides the start signal for a time-to-digital converter (TDC). The appropriately delayed, discriminated PMT signals from each end of the two scintillators provide the TDC stop signals. Note that the stage of additional delay represented by the "delay boxes" highlighted in pink in the diagram is required to provide sufficient dynamic range in the TDC to accommodate events in which the timing of the "scintillator 1 left" signal lags the timing of one or more of the other scintillator ends. Also note that the TDC stop signal for scintillator 1 "left" end is simply a constant-time delayed copy of the start signal. Thus, the TDC variable, representing the time difference between stop and start pulses, for this particular scintillator end is simply a constant (neglecting very small timing jitter effects).

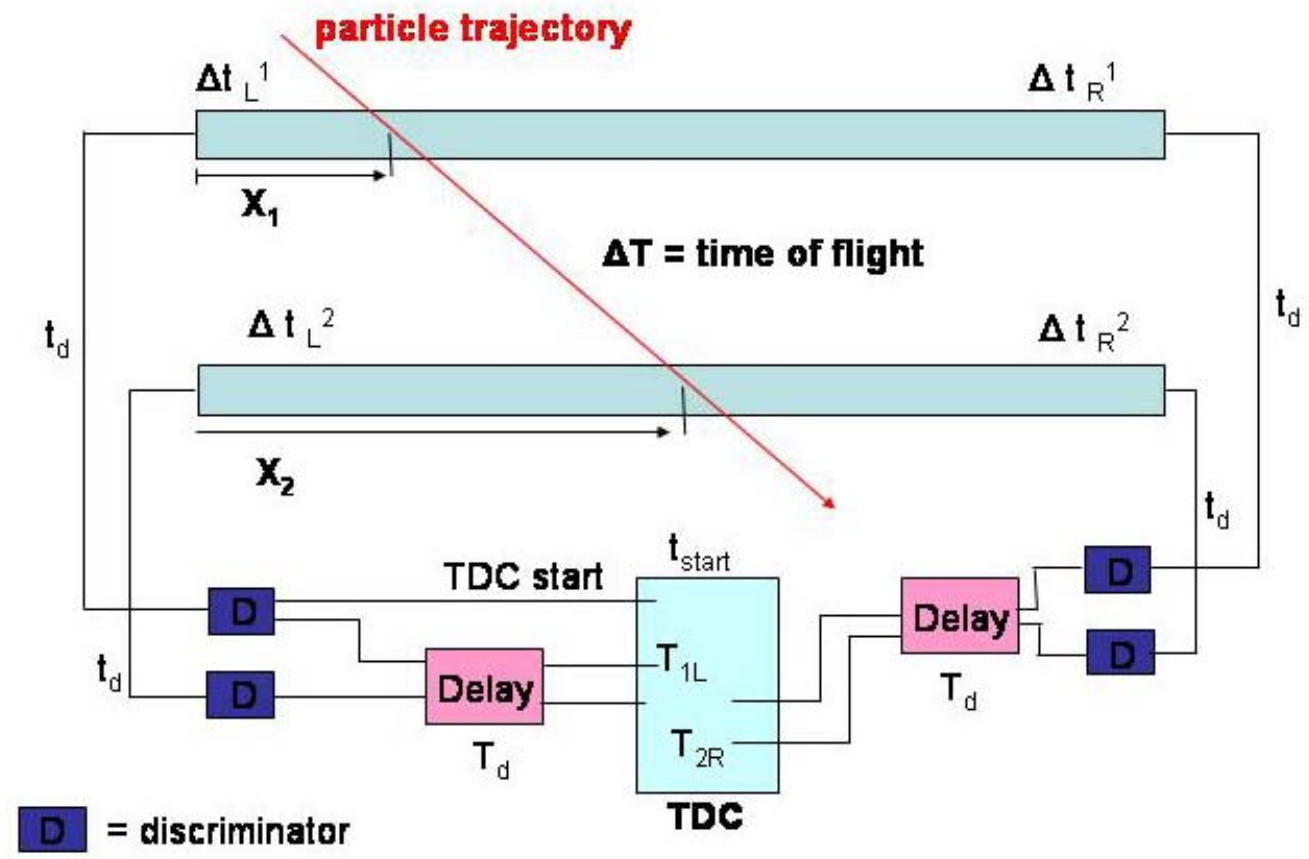

Figure 2.4. Conceptual Sensor Schematic Defining Timing Variables for Particle TOF and Interaction Position Reconstruction

It can be shown that the particle TOF through the sensor is given by the difference in the two scintillator mean times via 


$$
\mathrm{TOF}=\Delta \mathrm{T}=\frac{1}{2}\{\mathrm{TDC}(2, \mathrm{~L})+\mathrm{TDC}(2, \mathrm{R})-[\mathrm{TDC}(1, \mathrm{~L})+\mathrm{TDC}(1, \mathrm{R})]\}
$$

where $\operatorname{TDC}(i, j)$ refers to the TDC value registered for end $j(j=L, R)$ of scintillator $i(i=1,2)$. Similarly, the interaction positions $\mathrm{X}_{\mathrm{i}}$ can, in principle, be reconstructed from the TDC information and knowledge of the light propagation speed in the scintillator as follows:

$$
\begin{aligned}
\Delta t_{L}^{1} & =X_{1} / V_{\text {light }}, \quad \Delta t_{R}^{1}=\left(L-X_{1}\right) / V_{\text {light }} \\
X_{1} & =\frac{L-V_{\text {light }}\left(\Delta t_{R}^{1}-\Delta t_{L}^{1}\right)}{2} \\
& =\frac{L-V_{\text {light }}[T D C(1, R)-\operatorname{TDC}(1, L)]}{2}
\end{aligned}
$$

where the stand-alone term $\mathrm{L}$ in this set of equations is the length of the scintillator in the $\mathrm{X}$-axis direction. A similar expression can be obtained for $\mathrm{X}_{2}$. From the interaction positions $\mathrm{X}_{1}$ and $\mathrm{X}_{2}$, a correction to the nominal (i.e., normal-incidence) flight path through the sensor can be calculated, and the particle velocity through the sensor can then be obtained from the ratio of the flight path to the TOF. This analysis assumes that the effective signal propagation times to the two scintillator ends is governed solely by "line-of-sight" distances along the X-direction from the particle interaction point to the PMTs at each end. A fundamental requirement of the sensor readout electronics for this project is that the particle TOF be obtainable in a straightforward fashion from the raw data. In addition, the possibility of determining the scintillator interaction positions offers the potential for calculating a position-corrected velocity variable as well.

A schematic of the data-acquisition system is shown in Figure 2.5. Signals from the four center PMTs were sent to discriminator modules (Phillips PS 705) followed by pulse stretchers and fanouts. One output from each of the fanouts was sent to a four-fold coincidence logic module (Ortec CO4020). The data-acquisition system accepted only those events where valid timing signals were observed from all four of the center PMTs. Another output from each of the fanouts passed through an adjustable delay of about $240 \mathrm{~ns}$ and was sent to the Stop input of a multi-channel TDC. The TDC was triggered by a Common Start signal generated by a coincidence between a fanout output of one of the PMTs on the Front scintillator and the four-fold coincidence output signal. This trigger system meant that all time measurements were relative to the same PMT for every event. The four-fold coincidence was also used to generate a Veto signal that prevented the discriminators from accepting any other inputs for a period of $1.7 \mu$ s after the coincidence. Not shown in Figure 2.5 are three scalers that recorded the number of 4-fold coincidences, the number of 2-fold coincidences from the Front scintillator, and the number of 2-fold coincidences from the Back scintillator. 


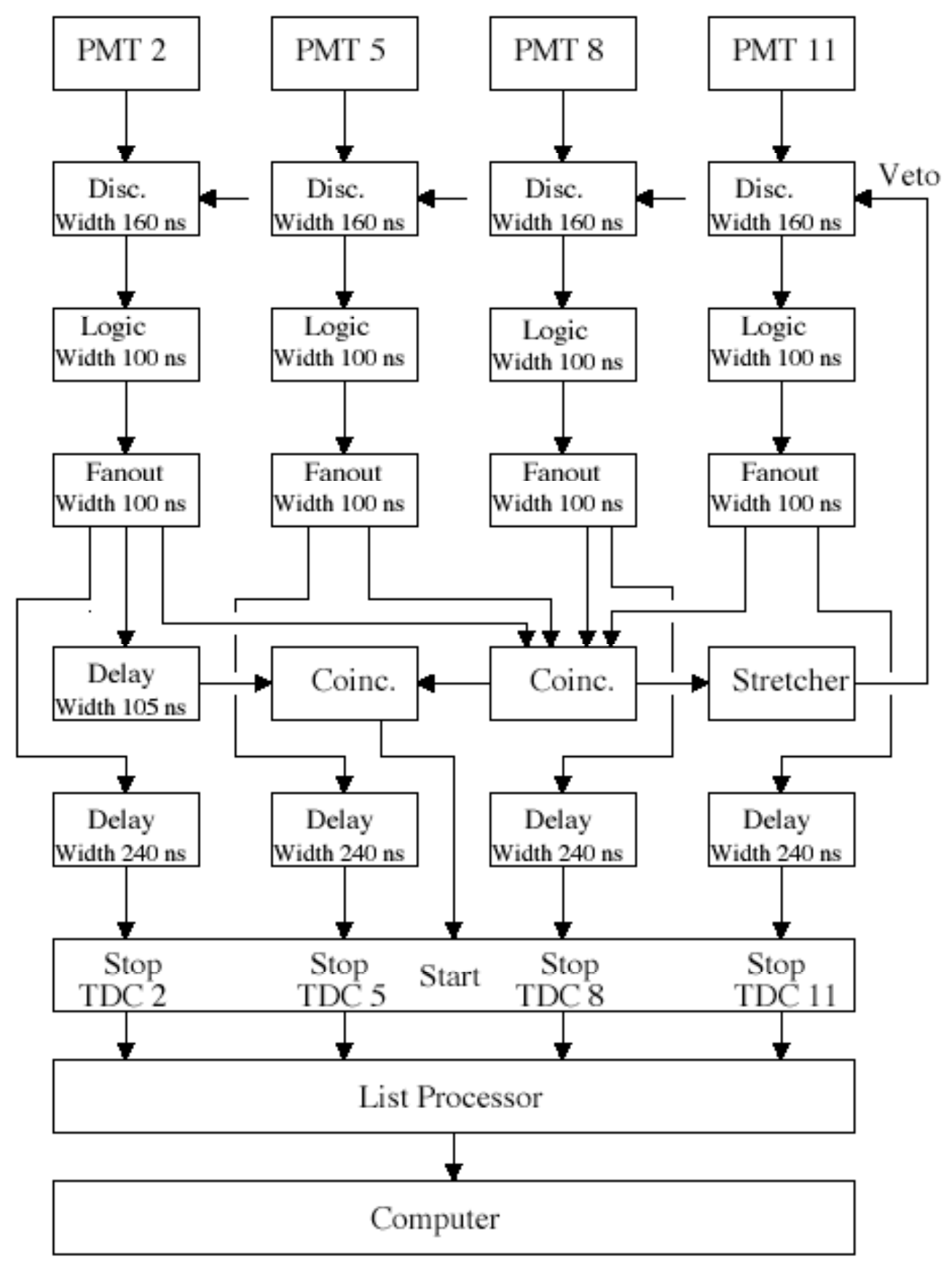

Figure 2.5. Schematic of Data-Acquisition System

The four PMTs were arbitrarily identified as PMT2 and PMT5 on the Front scintillator and PMT8 and PMT11 on the Back scintillator. The adjustable delays were set to put zero time at the same channel in the middle of 4096-channel histograms of the TDC outputs for all four PMTs. The calibration of the TDCs was $0.05 \mathrm{~ns} / \mathrm{ch}$ so that the total time range was $200 \mathrm{~ns}$. Because the Common Start trigger was generated by PMT 2, the histogram of its TDC pulses was an extremely narrow peak centered at time zero (channel 2000). The time distributions for the other three PMTs were broadly distributed above and 
below time zero. Figure 2.6 shows the time distributions for the four TDC outputs for the ${ }^{252} \mathrm{Cf}$ and ${ }^{137} \mathrm{Cs}$ sources at a discriminator setting of $100 \mathrm{mV}$.
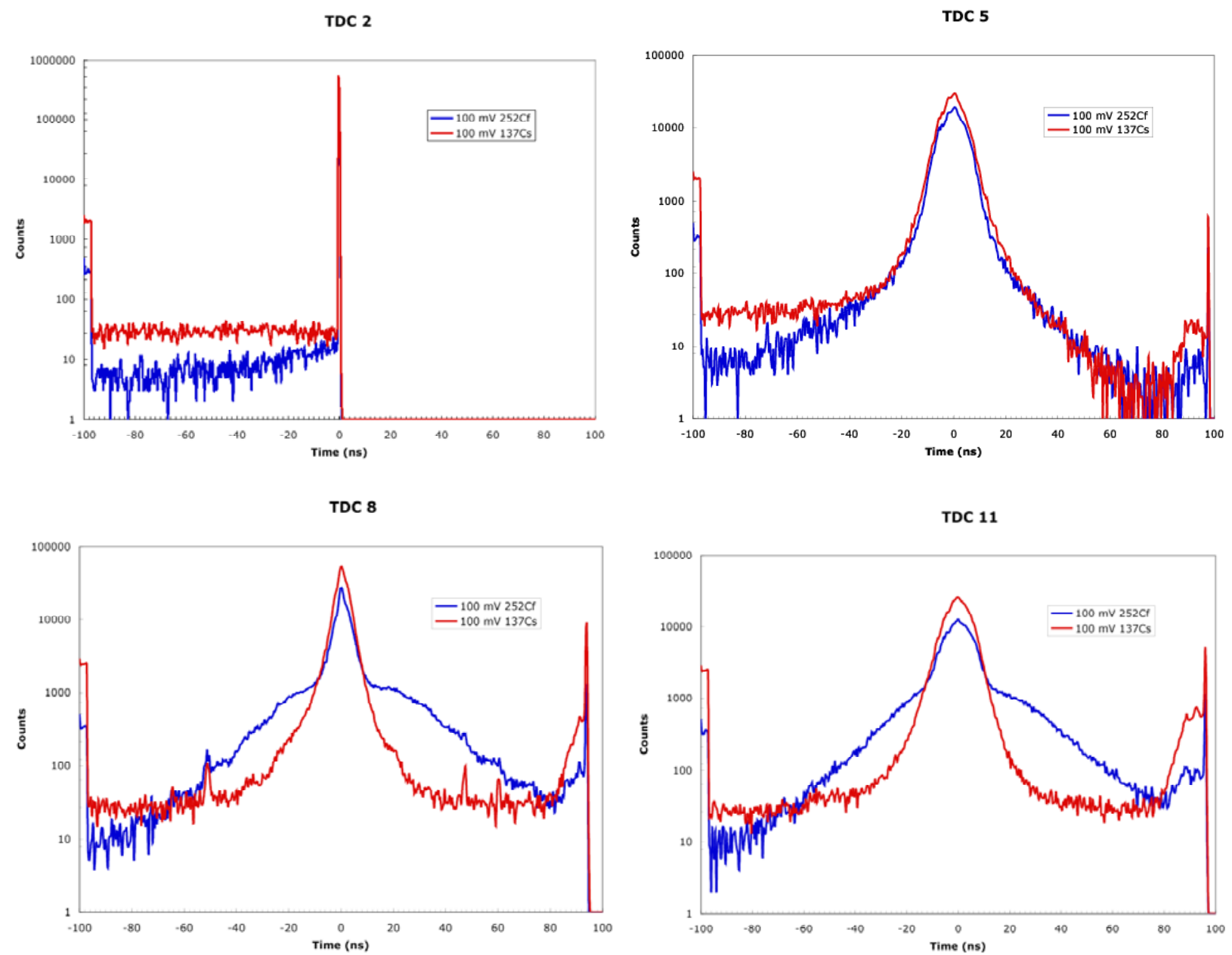

Figure 2.6. Time Distributions from Individual PMTs. Data binned at 0.4 ns per channel.

Because of the length of the scintillators, the transit time of a light pulse from one end to the other might be as large as $10 \mathrm{~ns}$, assuming the speed of light in the scintillator is 0.6 times the speed of light in air or $18 \mathrm{~cm} / \mathrm{ns}$. If an event in the front scintillator occurred close to PMT2, the arrival time of the light at PMT5 would be after the PMT2 pulse (positive time interval). If the event occurred close to PMT5, the light pulse at PMT5 arrives before the light pulse at PMT2 (negative time interval). Likewise pulses in PMTs 8 and 11 in the back scintillator could occur before or after the pulse in PMT2. For gamma rays, the transit time between the Front and Back scintillators could be as short as $0.5 \mathrm{~ns}$, which is significantly shorter than the transit time of light from one end of the scintillator to the other. For the neutron energies of interest here, the transit time between scintillators was generally longer than the transit time of light in the scintillator. In both cases, the TOF for the gamma or neutron was calculated as the difference between the average of the PMT2 and PMT5 times and the average of the PMT8 and PMT11 times. 
The data-acquisition system was based on a commercial multiparameter software package. ${ }^{\text {(a) }}$ The raw data (TOF for each PMT) were stored on an event-by-event basis in a list-processor (Hytec LP1342). When the list-processor memory was full, a block transfer of the data to the workstation computer was performed. The raw data could then be stored in event-mode files on the computer hard drive for off-line processing. In addition, the event-mode data could be processed by the acquisition computer between list-processor transfers to create histograms of raw and calculated parameters.

\subsubsection{Measurements}

The sources were mounted on the steel shield at the vertical and horizontal midpoint of the scintillators. Measurements of the TOF spectra were made at each of four discriminator settings $(10,20,50$, and $100 \mathrm{mV}$ ) for each of the sources. A few measurements were made with the sources at a distance of $62.2 \mathrm{~cm}$ from the steel sheet. The distance from the outside of the steel sheet to the front face of the Front scintillator was $12.7 \mathrm{~cm}$, corresponding to a solid angle of $35 \%$ of $4 \pi$. The solid angle for the sources at $62.2 \mathrm{~cm}$ from the steel sheet was calculated as $8.4 \%$ of $4 \pi$.

Background measurements were performed at every discriminator setting either before or following the source measurements at that setting. Count rates were low enough that no deadtime corrections were required.

\subsection{Results}

Both the background and source TOF histograms were normalized to $1000 \mathrm{~s}$ counting times. The background spectra and the net spectra after background subtraction given below were converted from channel number to time in ns based on the calibration of $0.05 \mathrm{~ns} / \mathrm{ch}$. Time zero is defined as if the average time in the Front scintillator and the average time in the Back scintillator were identical.

\subsubsection{TOF Spectra}

\subsubsection{Background}

Figure 2.7 shows the normalized background spectra for two different discriminator levels. The time range shown is dominated by gamma-type events. The spike at time zero is an artifact related to events where all four PMTs had a 0 TDC channel. The spectra decrease in amplitude as the discriminator is raised. The double peak can be explained as the result of events where the first Compton scattering occurred in the Front scintillator (positive times) or in the Back scintillator (negative times). The sensor's background response is almost certainly dominated by cosmic rays that have an essentially equal probability of traversing the sensor in the "front to back" and "back to front" directions. It is also possible that some terrestrial gammas entering from the front pass through the Front scintillator without interacting, but then interact in the Back scintillator and scatter backwards into the Front scintillator. The lead shield on four sides protects the Back scintillator better than the Front scintillator, so the Front scintillator is more susceptible to gammas coming from the local environment. Thus, the intensity of the Background TOF spectrum favors those events that originate in the Front scintillator.

(a) KMAX version 7.3g9, Sparrow Corp., Port Orange, FL 32128. 


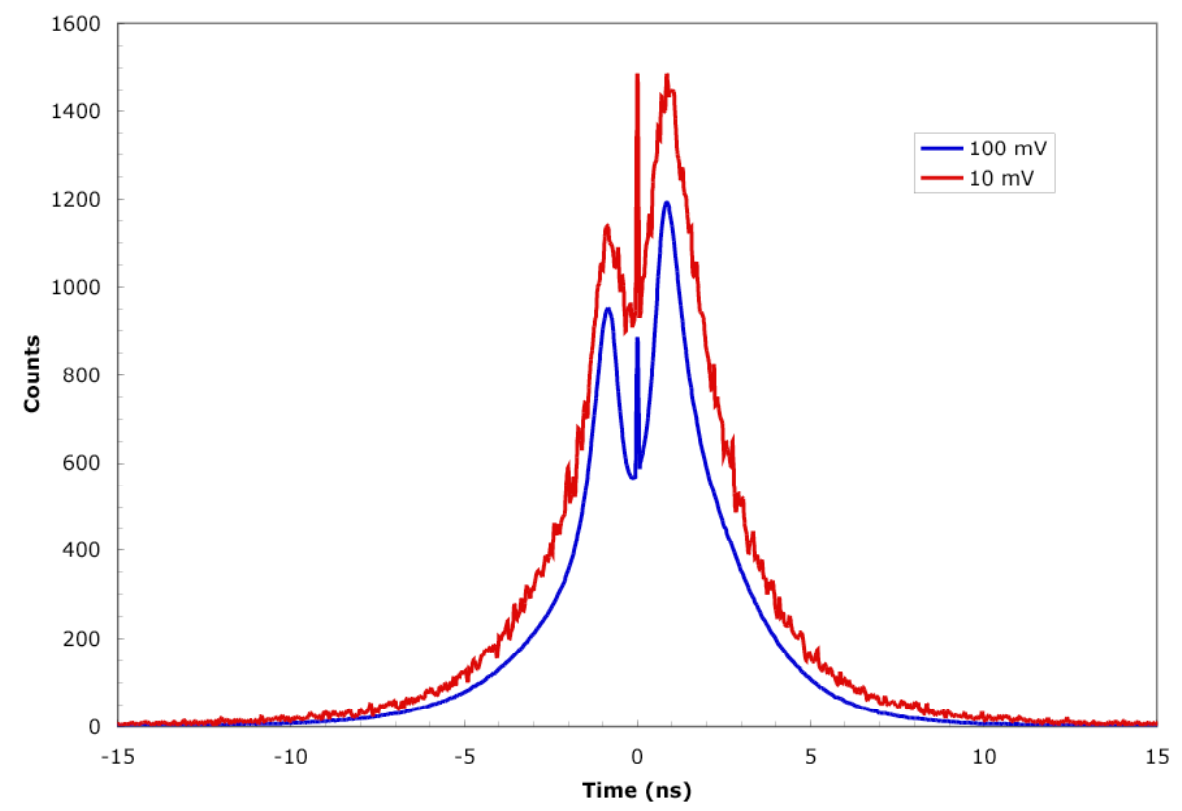

Figure 2.7. TOF Spectra for the Background at Two Different Discriminator Levels

\subsubsection{Gamma}

Figure 2.8 shows the net gamma TOF spectra for the ${ }^{137}$ Cs source for two discriminator levels. Again the count rate decreases as the discriminator level is raised.

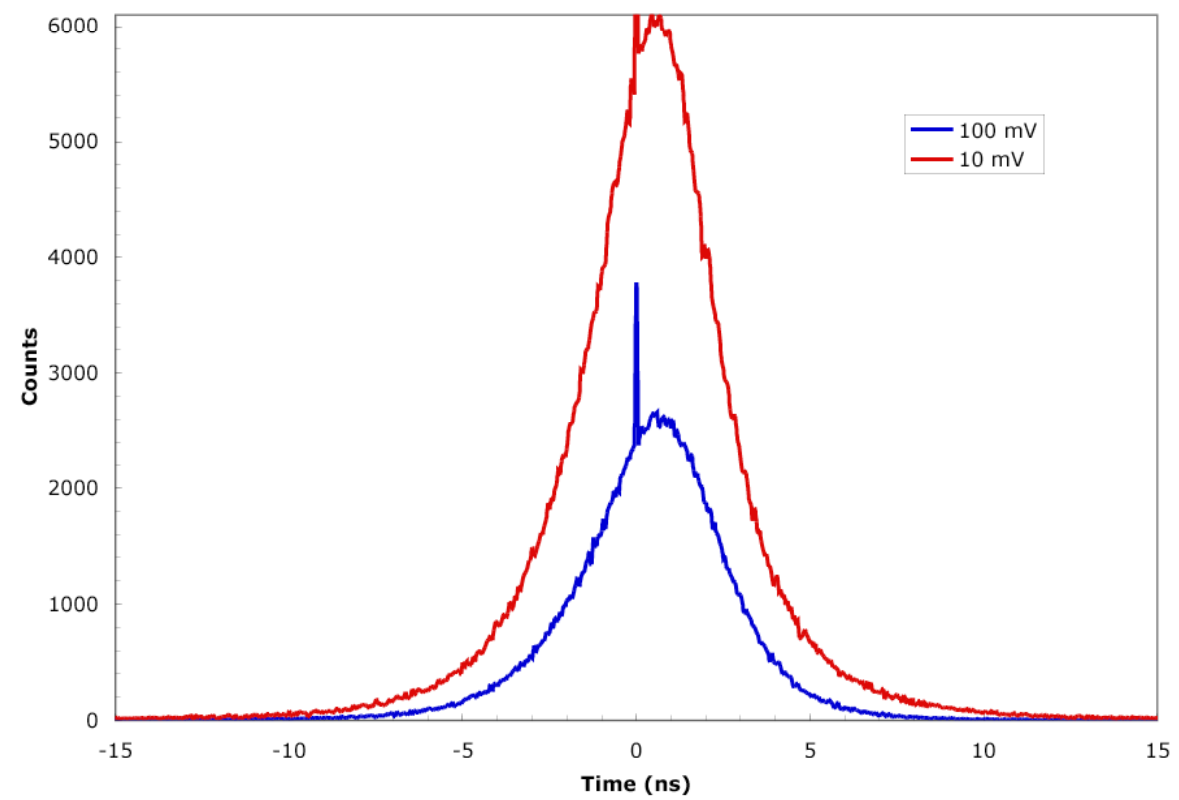

Figure 2.8. Comparison of ${ }^{137} \mathrm{Cs}$ Gamma TOF Spectra for the Source Mounted at the Midpoint of the Steel Shield for Two Discriminator Levels 
The net gamma TOF spectra do not show the double peak. This indicates that the TOF spectrum is dominated by events that first interact in the Front scintillator when the gamma source is in front of the detector assembly. Note that the peak of the gamma TOF spectrum is shifted by about $0.5 \mathrm{~ns}$ from time zero. This is consistent with the expected transit time for a gamma traveling the shortest possible distance between the scintillators $(13 \mathrm{~cm})$ at the velocity of light in $\operatorname{air}(30 \mathrm{~cm} / \mathrm{ns})$.

Figure 2.9 shows a comparison of the gamma TOF spectra at two distances for the discriminator level of $100 \mathrm{mV}$. Figure 2.10 shows similar data for the discriminator at $10 \mathrm{mV}$. Although the count rates are reduced at the farther distance, the shape of the spectra is the same.

The possibility that gamma TOF spectra might depend somewhat on the energy of the incident gamma ray was investigated by comparing TOF spectra for three gamma sources, ${ }^{137} \mathrm{Cs}(662 \mathrm{keV}),{ }^{54} \mathrm{Mn}$ $(835 \mathrm{keV})$, and ${ }^{60} \mathrm{Co}(1173-$ and $1332-\mathrm{keV})$. Figure 2.11 gives a comparison of normalized TOF spectra for ${ }^{137} \mathrm{Cs}$ and ${ }^{60} \mathrm{Co}$ at two different discriminator settings. There is very little difference in the shape of the TOF spectra as a function of gamma energy at either discriminator level. However, the discriminator level affects the detection efficiency for the ${ }^{137} \mathrm{Cs}$ source more than the ${ }^{60} \mathrm{Co}$ source in that the higher discriminator level requires a higher normalization factor for ${ }^{137} \mathrm{Cs}$ (ratio of ${ }^{60} \mathrm{Co}$ peak counts to ${ }^{137} \mathrm{Cs}$ peak counts $=4.05$ at $10 \mathrm{mV}$ and ratio of ${ }^{60} \mathrm{Co}$ peak counts to ${ }^{137} \mathrm{Cs}$ peak counts $=6.50$ at $100 \mathrm{mV}$ ).

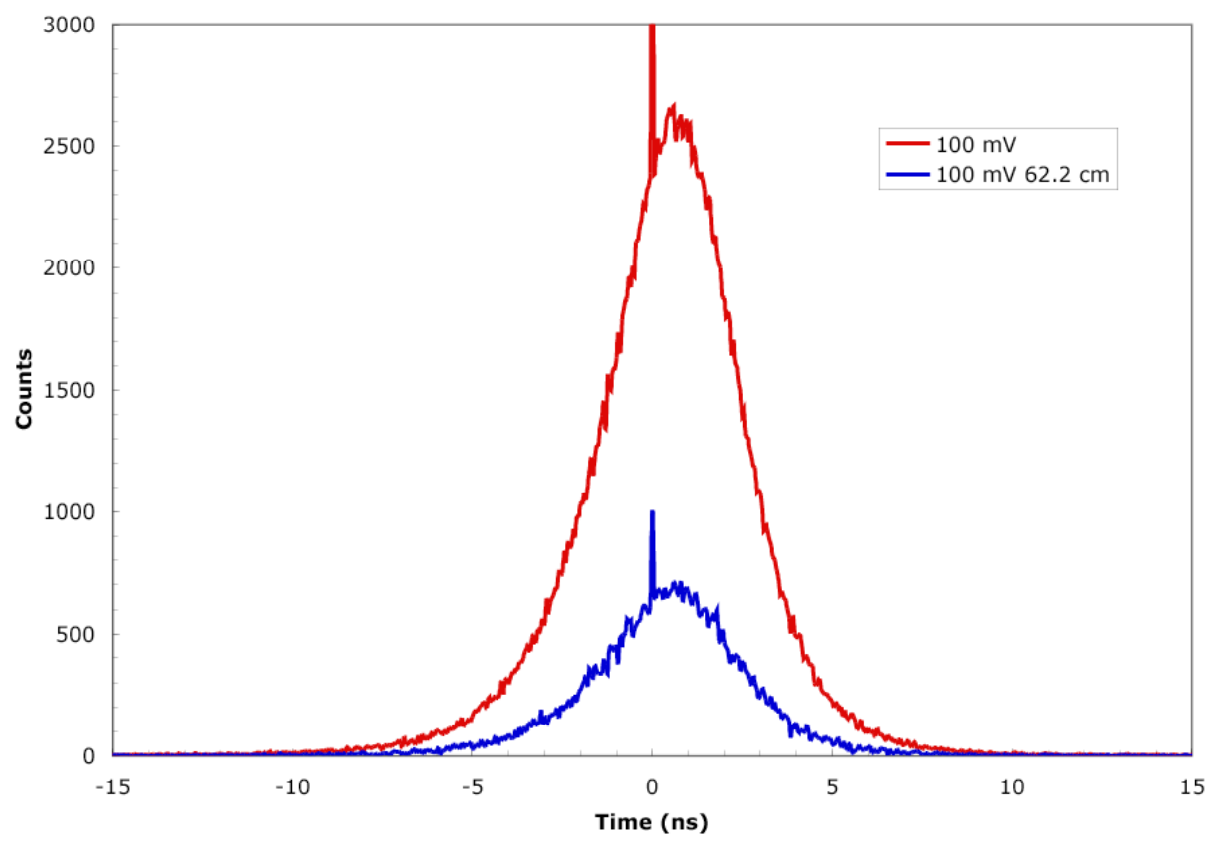

Figure 2.9. Comparison of ${ }^{137} \mathrm{Cs}$ Gamma TOF Spectra at a Discriminator Level of $100 \mathrm{mV}$ for the Standard Location and a Location 62.2-cm Farther Away 


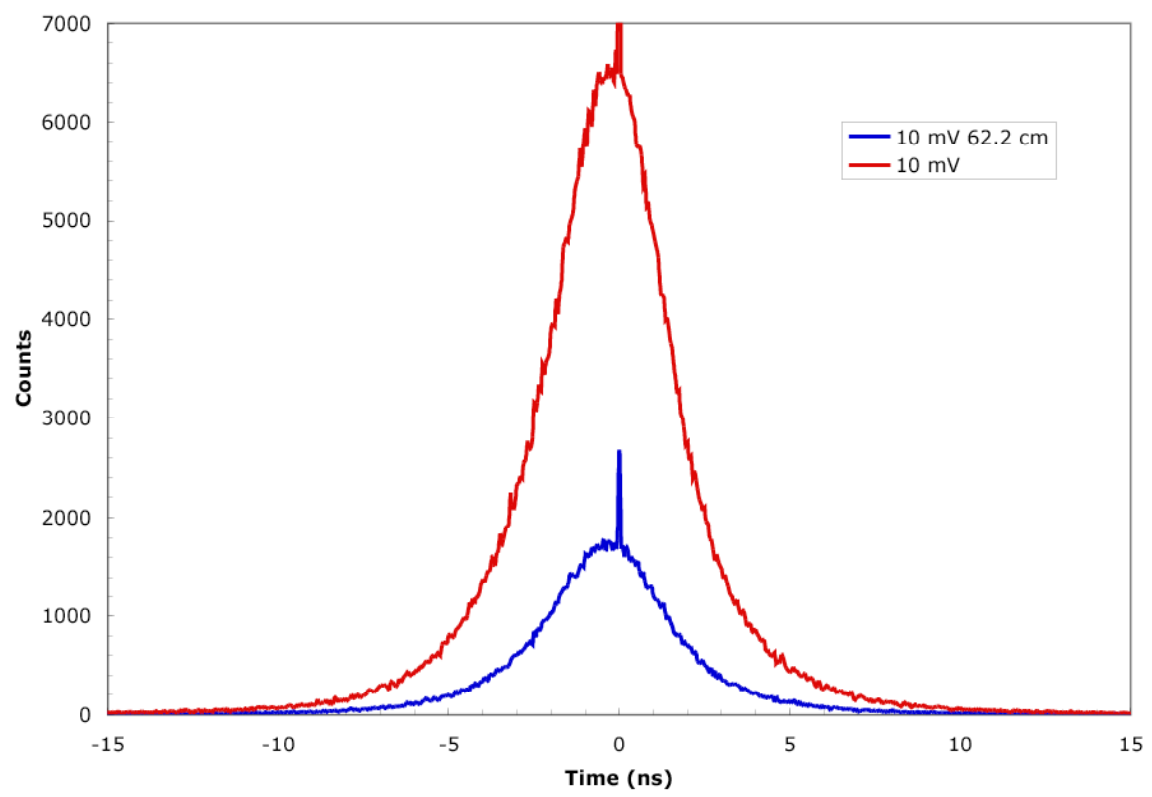

Figure 2.10. Comparison of ${ }^{137} \mathrm{Cs}$ Gamma TOF Spectra at a Discriminator Level of $10 \mathrm{mV}$ for the Standard Location and a Location 62.2-cm Farther Away. The shift in peak position relative to the time 0 peak is caused by different arbitrary time offsets for the TDC data relative to previously shown data.
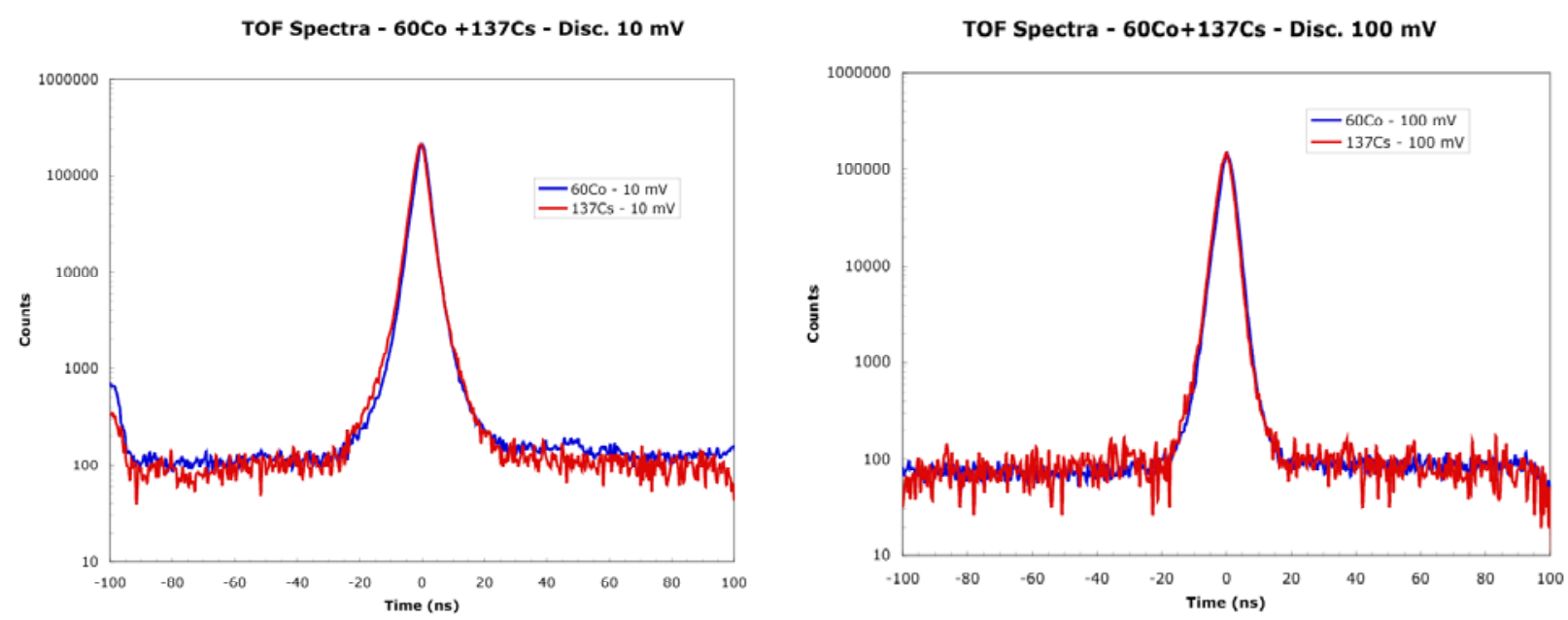

Figure 2.11. Comparison of Gamma TOF Spectra for Different Energy Gamma Sources at Two Discriminator Levels

The discriminator level has a significant effect on the shape of the TOF spectra as illustrated in Figure 2.12, which shows normalized TOF spectra for the ${ }^{60} \mathrm{Co}$ source at two discriminator settings. The TOF spectrum is narrower at the higher discriminator level. Because of the minimal dependence of the TOF spectra on gamma energy, the detailed analysis of gamma fraction and efficiency above a given TOF cutoff discussed below is based primarily on the ${ }^{137} \mathrm{Cs}$ data. 


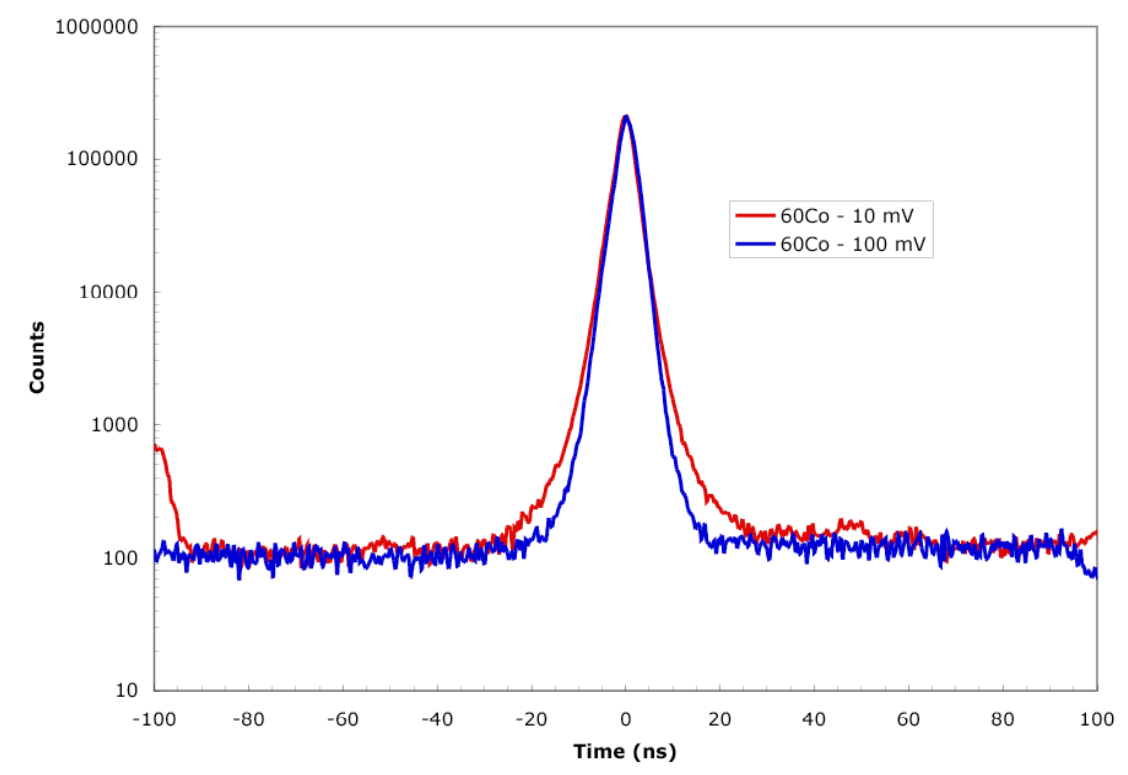

Figure 2.12. Comparison of ${ }^{60} \mathrm{Co}$ TOF Spectra at Two Discriminator Levels

\subsubsection{Neutron}

Figure 2.13 shows the net TOF spectra for the ${ }^{252} \mathrm{Cf}$ source. Because of lower count rates, the TOF data have been binned in channels of $0.4 \mathrm{~ns}$. The ${ }^{252} \mathrm{Cf}$ source emits gammas and neutrons so the backgroundsubtracted data include a narrow gamma distribution as well as the very broad neutron distribution extending out to $\pm 100 \mathrm{~ns}$. In contrast to the gamma data, the neutron TOF spectra show a significant distribution at negative times as well as at the expected positive times. This is understood as neutrons that do not interact with either scintillator before scattering off the lead shield. A neutron does not lose much energy in scattering from lead. The neutron can bounce back, interact in the Back scintillator, and then interact in the Front scintillator, thus producing a TOF spectrum with negative times.

The reasonableness of this interpretation of the negative TOF portion of the neutron response was demonstrated by placing the ${ }^{252} \mathrm{Cf}$ source on the backside of the detector assembly. The neutrons pass through the lead shield, interact with the Back scintillator, and then interact with the Front scintillator, giving a TOF spectrum at negative times. There is no lead on the front side of the detector assembly, so there is no back-scattering to produce a corresponding TOF spectrum at positive times. Figure 2.14 shows the experimental TOF spectrum with the discriminator set at $100 \mathrm{mV}$. The greatly reduced TOF spectrum at positive times confirms the expectations regarding lead back-scattering. 


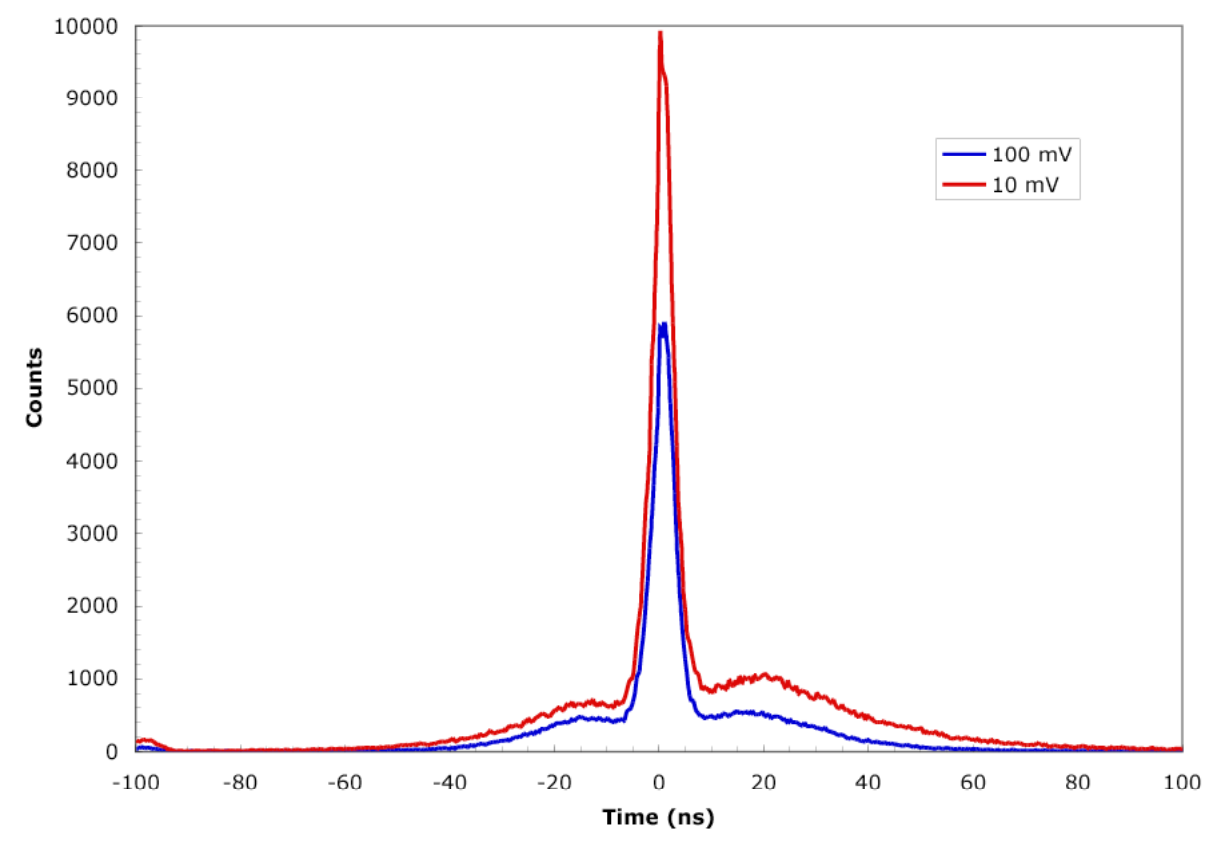

Figure 2.13. Comparison of Net ${ }^{252} \mathrm{Cf}$ TOF Spectra After Normal Background Subtraction for the Source Mounted at the Midpoint of the Steel Shield for Two Discriminator Levels

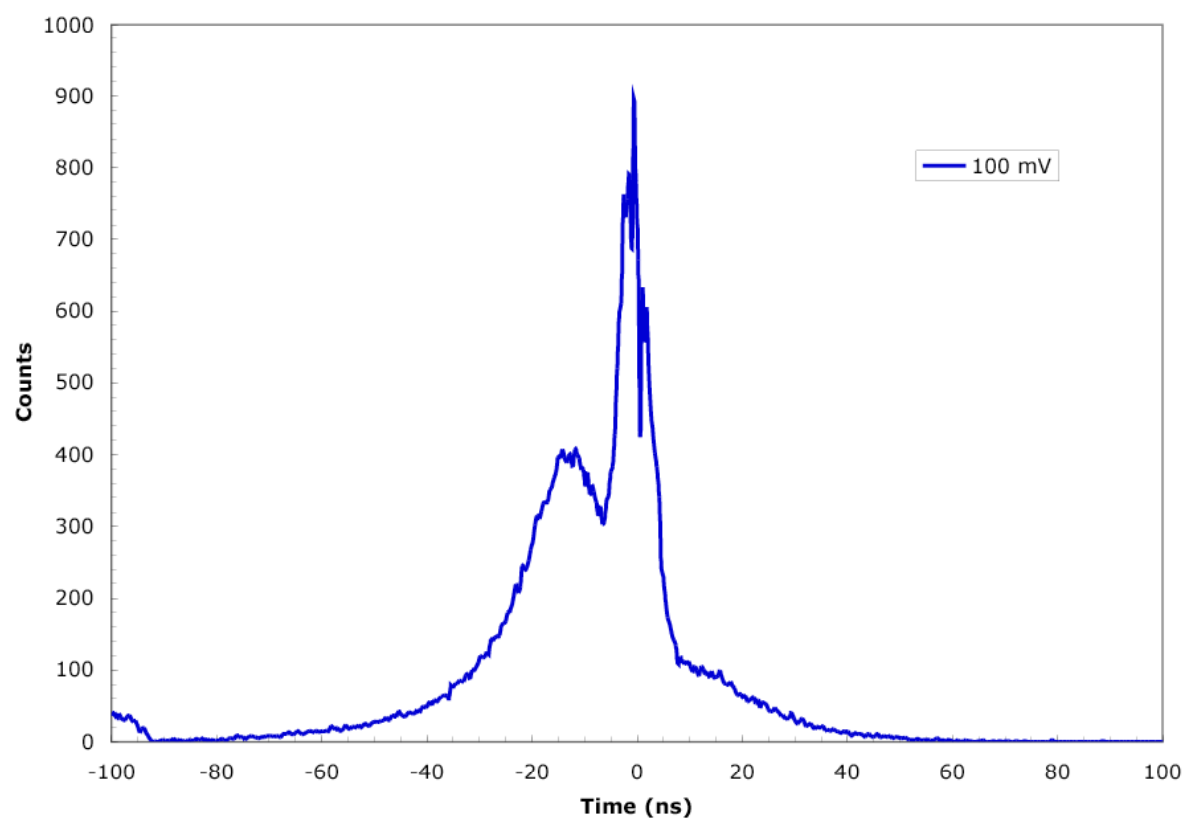

Figure 2.14. Net ${ }^{252} \mathrm{Cf}$ TOF Spectra After Normal Background Subtraction for Source Mounted Outside the Lead Shield at the Midpoint of the Backside of the Detector Housing. Note the reduced neutron TOF data at positive times. Also note the reduced gamma peak because of the 5-cm thick lead shield. 
To isolate the sensor's fast neutron TOF response, a procedure was used to strip out a gamma distribution having a standard shape from each ${ }^{252} \mathrm{Cf}$ TOF spectrum. The standard shape was determined by fitting the ${ }^{137} \mathrm{Cs}$ gamma TOF spectra with a function that was Gaussian at the peak. Away from the peak, the gamma distribution was fit with the sum of two exponential components. This functional form was fitted to the gamma region of the ${ }^{252} \mathrm{Cf}$ TOF spectra. The width of the Gaussian distribution was about $10 \%$ greater for gammas from ${ }^{252} \mathrm{Cf}$ than for gammas from ${ }^{137} \mathrm{Cs}$. The slopes of the two exponentials were the same in both cases. The peak of the gamma distribution from ${ }^{252} \mathrm{Cf}$ was at about $1 \mathrm{~ns}$ rather than at $0.5 \mathrm{~ns}$ as in the ${ }^{137} \mathrm{Cs}$ spectrum. These shifts in fitting parameters may be because ${ }^{252} \mathrm{Cf}$ has a broad spectrum of energies whereas ${ }^{137} \mathrm{Cs}$ has a single peak at $662 \mathrm{keV}$. Another possible difference is that the gamma TOF spectra were binned at $0.05 \mathrm{~ns} / \mathrm{ch}$ whereas the neutron TOF data were binned at $0.4 \mathrm{~ns} / \mathrm{ch}$.

Figure 2.15 shows the net neutron TOF spectra after subtraction of the fitted gamma distribution. Note that the data between -5 and +5 ns are subject to large statistical uncertainties because of large background and gamma subtractions in this region. Note that as the discriminator is raised, the ratio of the total number of neutron events in the positive time region decreases relative to the total number of events in the negative time region. Figure 2.16 compares the neutron TOF spectra at two distances for the discriminator level of $100 \mathrm{mV}$. Figure 2.17 shows similar data for the discriminator at $10 \mathrm{mV}$. There is a strong reduction in the number of counts in the negative TOF region relative to the number in the positive TOF region at the farther distance. This is in contrast to the gamma TOF spectra, which had the same shape at both distances.

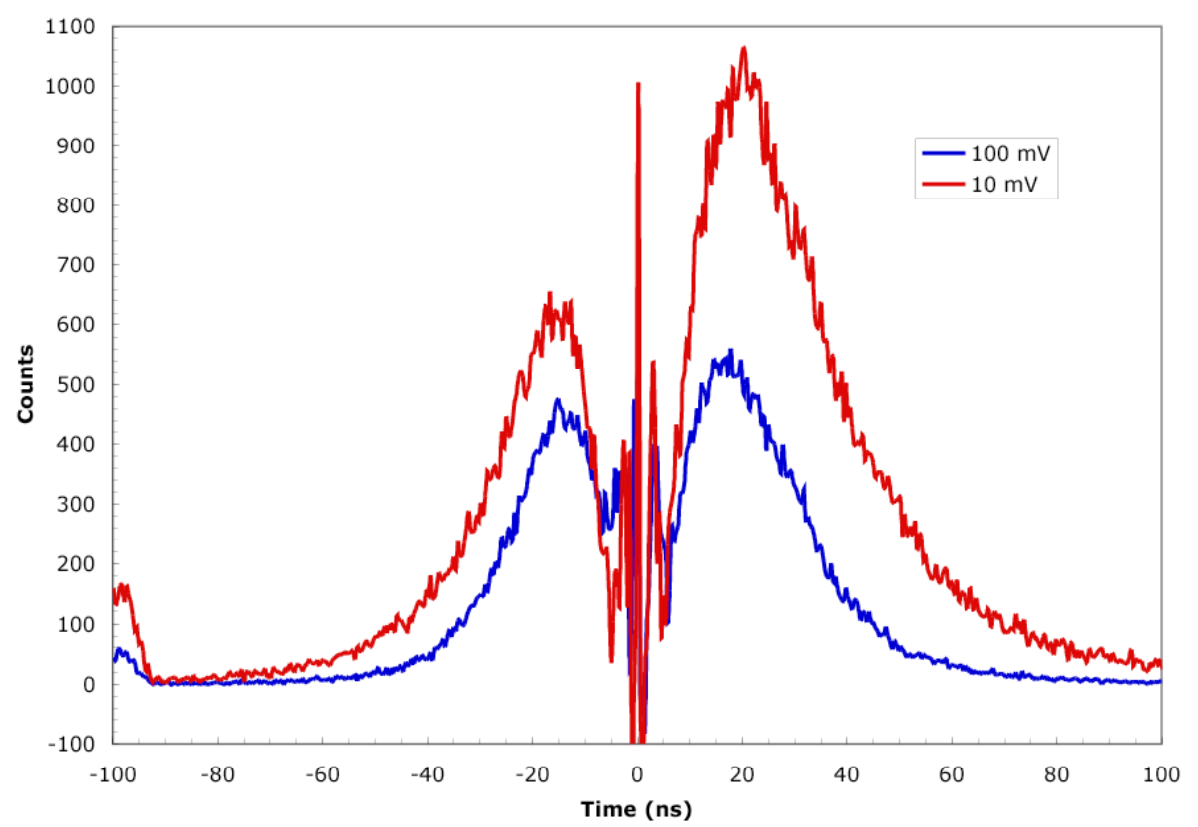

Figure 2.15. Comparison of net ${ }^{252} \mathrm{Cf}$ Neutron TOF Spectra After Subtraction of the ${ }^{252} \mathrm{Cf}$ Gamma Component for the Source Mounted at the Midpoint of the Steel Shield for Two Discriminator Levels 


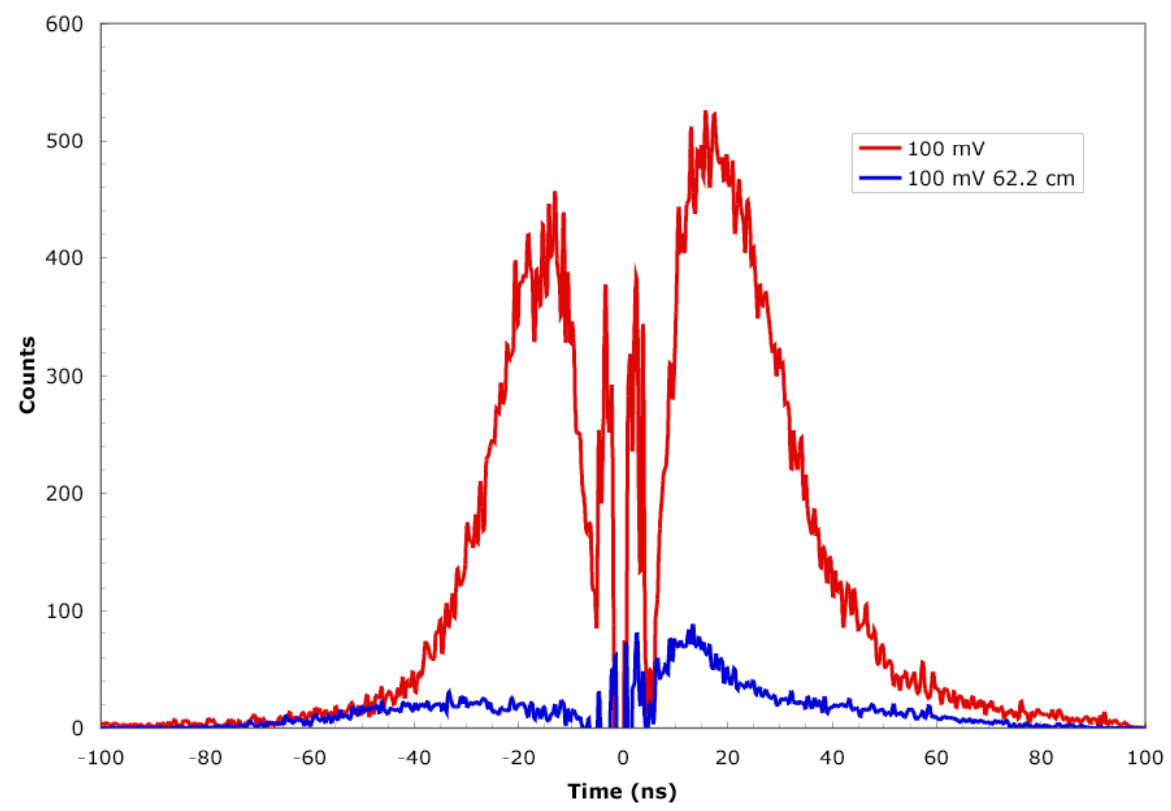

Figure 2.16. Comparison of Net ${ }^{252} \mathrm{Cf}$ Neutron TOF Spectra for the Normal Source Location and a Location 62.2-cm Farther Away (discriminator level $=100 \mathrm{mV}$ )

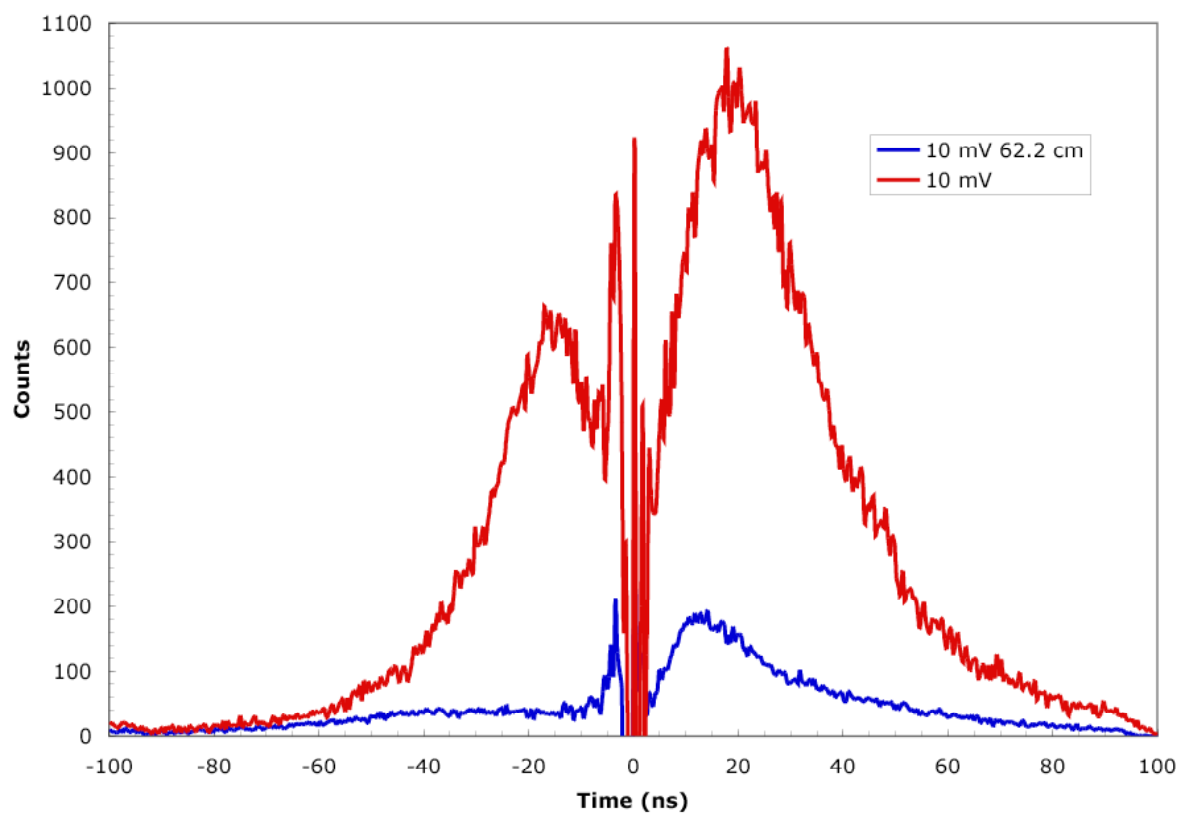

Figure 2.17. Comparison of Net ${ }^{252} \mathrm{Cf}$ Neutron TOF Spectra for the Normal Source Location and a Location 62.2-cm Farther Away (discriminator level $=10 \mathrm{mV}$ )

\subsubsection{Comparison of Neutron and Gamma TOF Spectra}

Figure 2.18 compares the TOF spectra for the neutron and gamma sources at the same discriminator level. In every case, the gamma events occur in a narrow time region between the positive and negative neutron time distributions. 


\subsubsection{Fractional Efficiencies}

\subsubsection{Gamma}

To understand the probability of gamma contamination above a given time threshold, the ratio of counts above a given time threshold to the total number of counts was calculated. This fractional efficiency was calculated separately for the gamma distribution above the peak (Forward) and the gamma distribution below the peak (Reverse). Note that the peak channel is not the time zero channel. No intrinsic difference between the Forward and Reverse distributions is expected, and the total gamma efficiency should be based on the sum of the entire peak. However, a comparison of the fractional efficiency in the Forward and Reverse distributions will reveal any possible asymmetry in the shape of the gamma TOF spectrum.
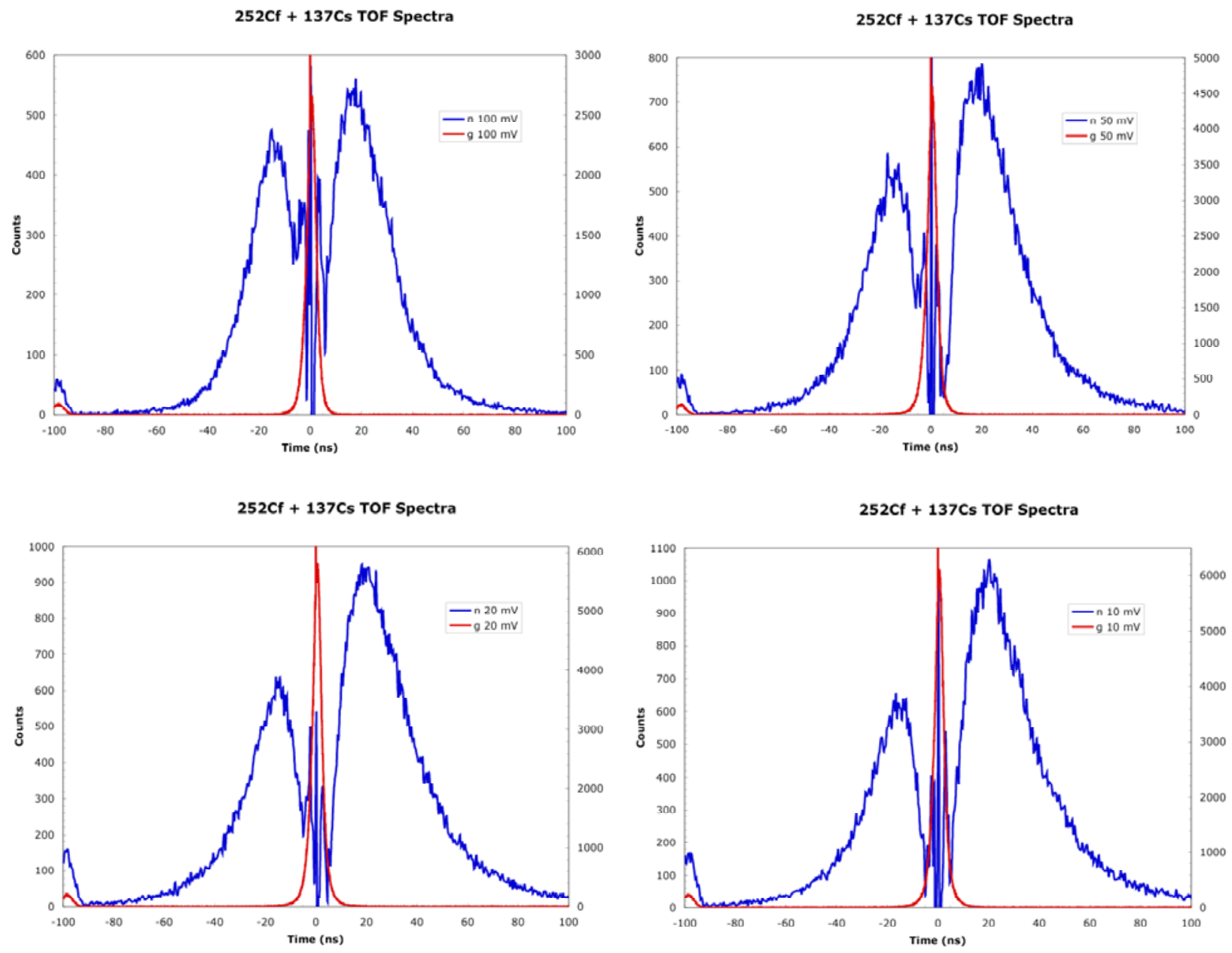

Figure 2.18. Comparison of the ${ }^{137} \mathrm{Cs}$ Gamma TOF Spectrum and the ${ }^{252} \mathrm{Cf}$ Neutron TOF Spectrum at each of the four Discriminator Levels. The source is in the normal location. The gamma counts scale is on the right edge of each figure. 
Figure 2.19 shows fractional efficiencies as a function of the time threshold for each of the discriminator settings. For the $10-\mathrm{mV}$ and $20-\mathrm{mV}$ discriminator levels, the Forward and Reverse fractional efficiencies are essentially the same. However, at the $50-\mathrm{mV}$ and $100-\mathrm{mV}$ discriminator levels, the fractional efficiency decreases more rapidly for the Forward distribution than the Reverse distribution. This indicates an asymmetry in the peak shape such that the Forward distribution becomes narrower at the higher discriminators.

Figure 2.20 shows fractional efficiencies for the Reverse and Forward distributions for all four discriminator levels. Both the Forward and Reverse distributions get narrower as the discriminator level is raised to $100 \mathrm{mV}$.
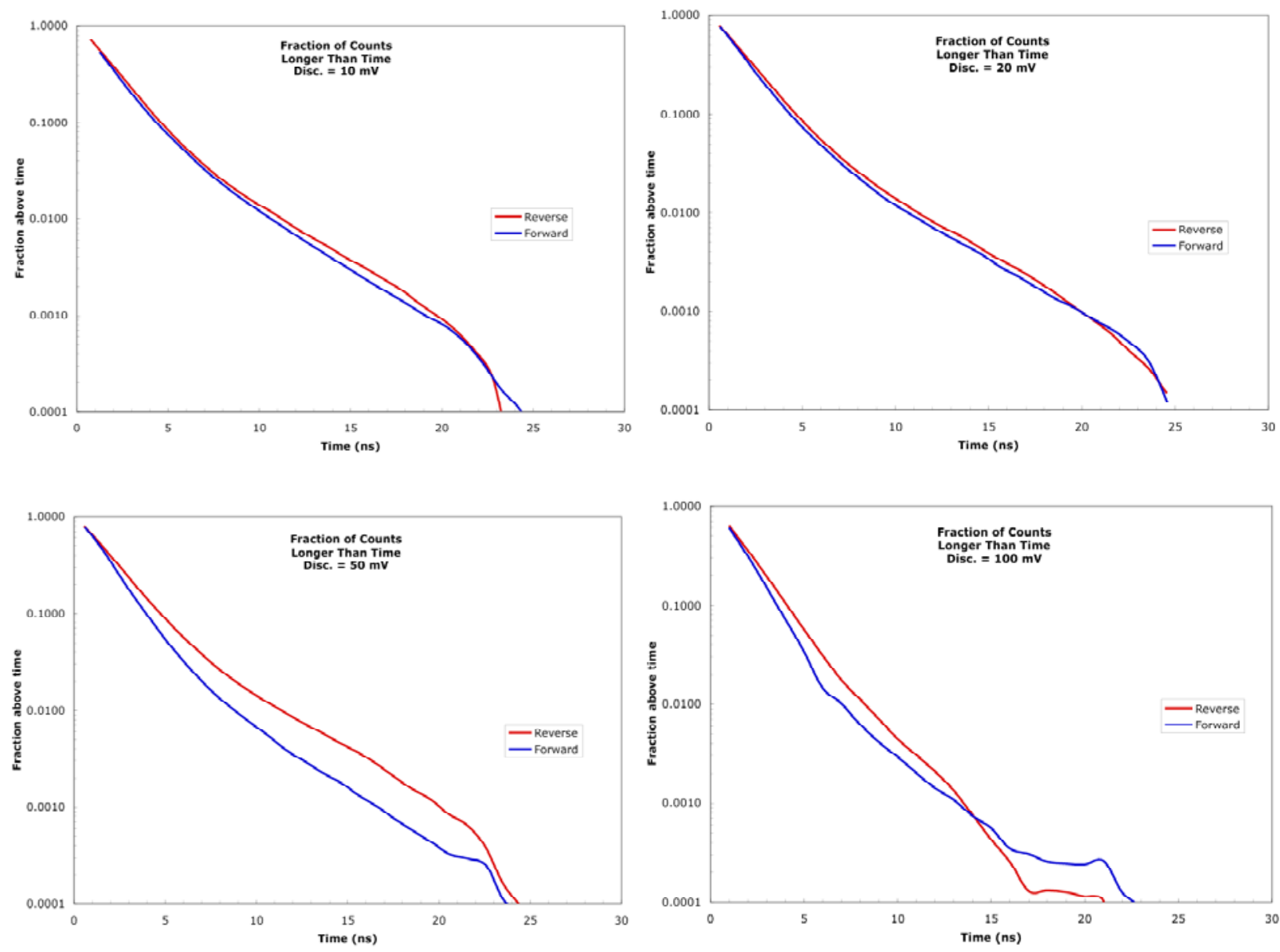

Figure 2.19. Fractional Efficiency for ${ }^{137} \mathrm{Cs}$ as a Function of Time Threshold at each of the four Discriminator Levels 

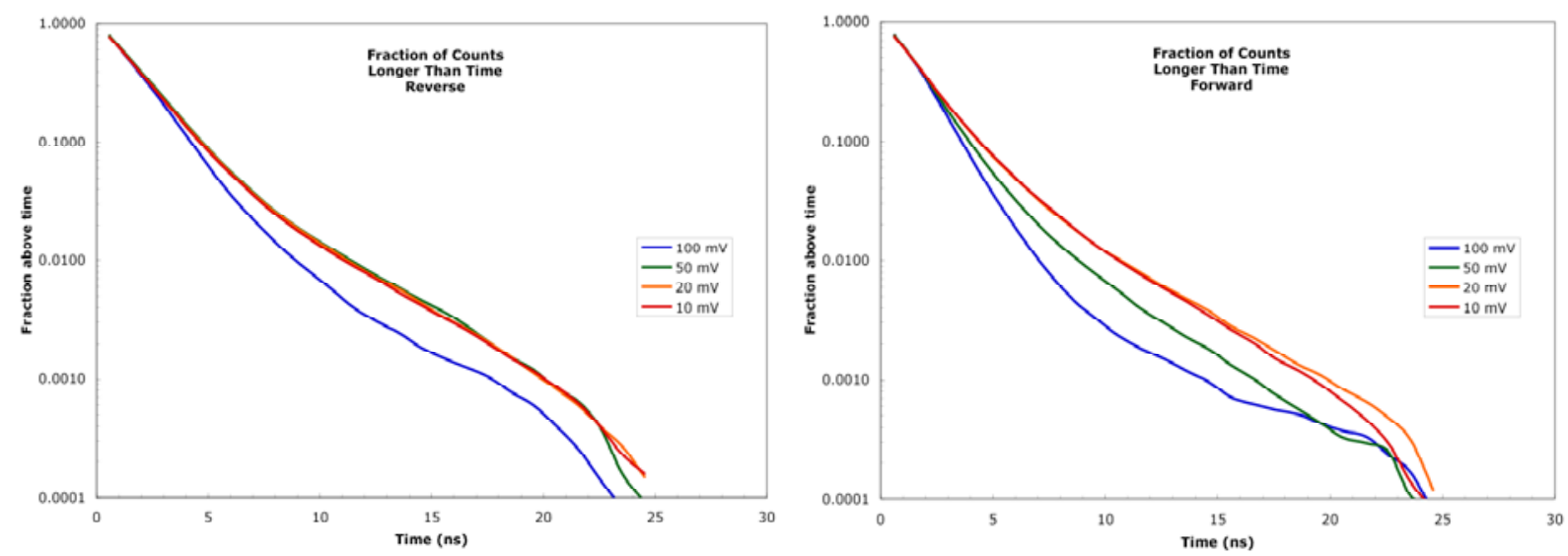

Figure 2.20. Comparison of Fractional Efficiency for ${ }^{137} \mathrm{Cs}$ in Reverse or Forward Distributions as a Function of Discriminator Levels

Fractional efficiencies at different source distances are shown for the discriminator levels at 10 and $100 \mathrm{mV}$ in Figure 2.21. The good agreement in the fractional efficiency curves between the data at different distances indicates that the shape of the spectra do not change because of the extra distance.
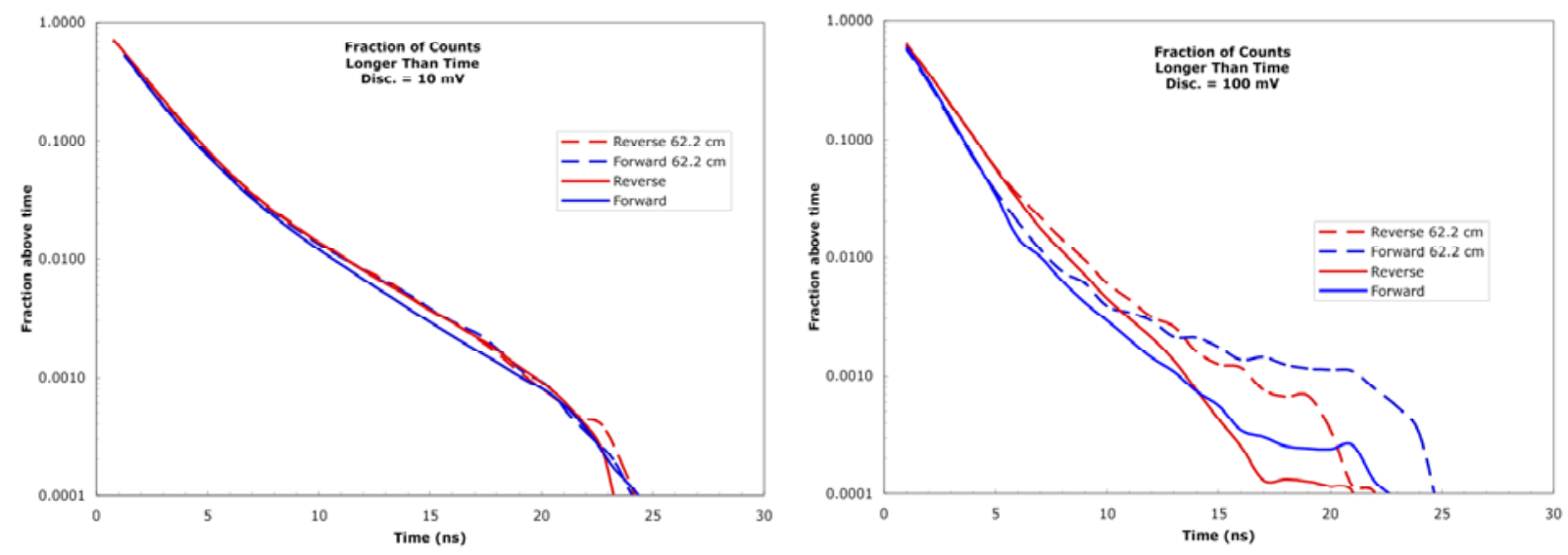

Figure 2.21. Fractional Efficiencies for ${ }^{137} \mathrm{Cs}$ at the Standard Source Distance and at a Distance 62.2-cm Farther Away

Figure 2.22 shows the relative efficiency of total counts as a function of discriminator for the Reverse, Forward, and Total gamma distributions. 


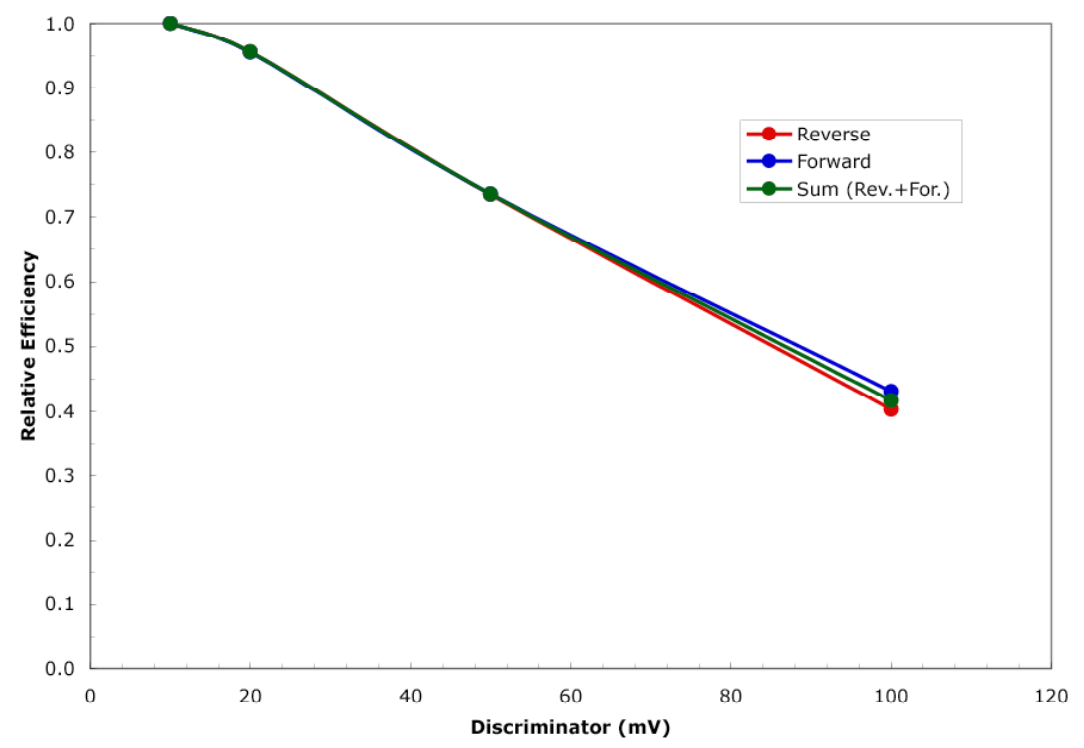

Figure 2.22. Relative Efficiency of Total Gamma Counts as a Function of Discriminator Level. Source at normal location.

\subsubsection{Neutron}

Fractional efficiencies for the neutron TOF spectra were calculated in a similar way as for the gamma fractional efficiency. In this case, however, the Forward fractional efficiency was based on positive time intervals only, and the Reverse fractional efficiency was based on negative time intervals only. The Forward and Reverse distributions are based on distinctly different scattering processes. As noted in section 2.2.1.3 above, the Reverse neutron response appears to be caused entirely, or at least predominantly, by the presence of the lead shield.

Figure 2.23 shows the fractional efficiencies for neutrons as a function of time threshold. Note that in these and subsequent figures, the neutron time intervals are relative to the peak of the ${ }^{252} \mathrm{Cf}$ gamma TOF spectrum and not the zero time channel. In every case, the Reverse distribution decreases more rapidly than the Forward distribution, indicating different spectral shapes.

Figure 4.24 shows fractional efficiencies for the Reverse and Forward distributions for all four discriminator levels. Both the Forward and Reverse distributions get narrower as the discriminator level is raised to 50 and $100 \mathrm{mV}$.

Fractional efficiencies at different source distances are shown for the discriminator level at $100 \mathrm{mV}$ in Figure 2.25. The lack of agreement in the fractional efficiency curves between the data at different distances indicates that the shape of the spectrum is quite different at the longer distance. In fact, the Forward distribution decreases more rapidly than the Reverse distribution at the longer distance whereas it is the exact opposite at the closer distance. 

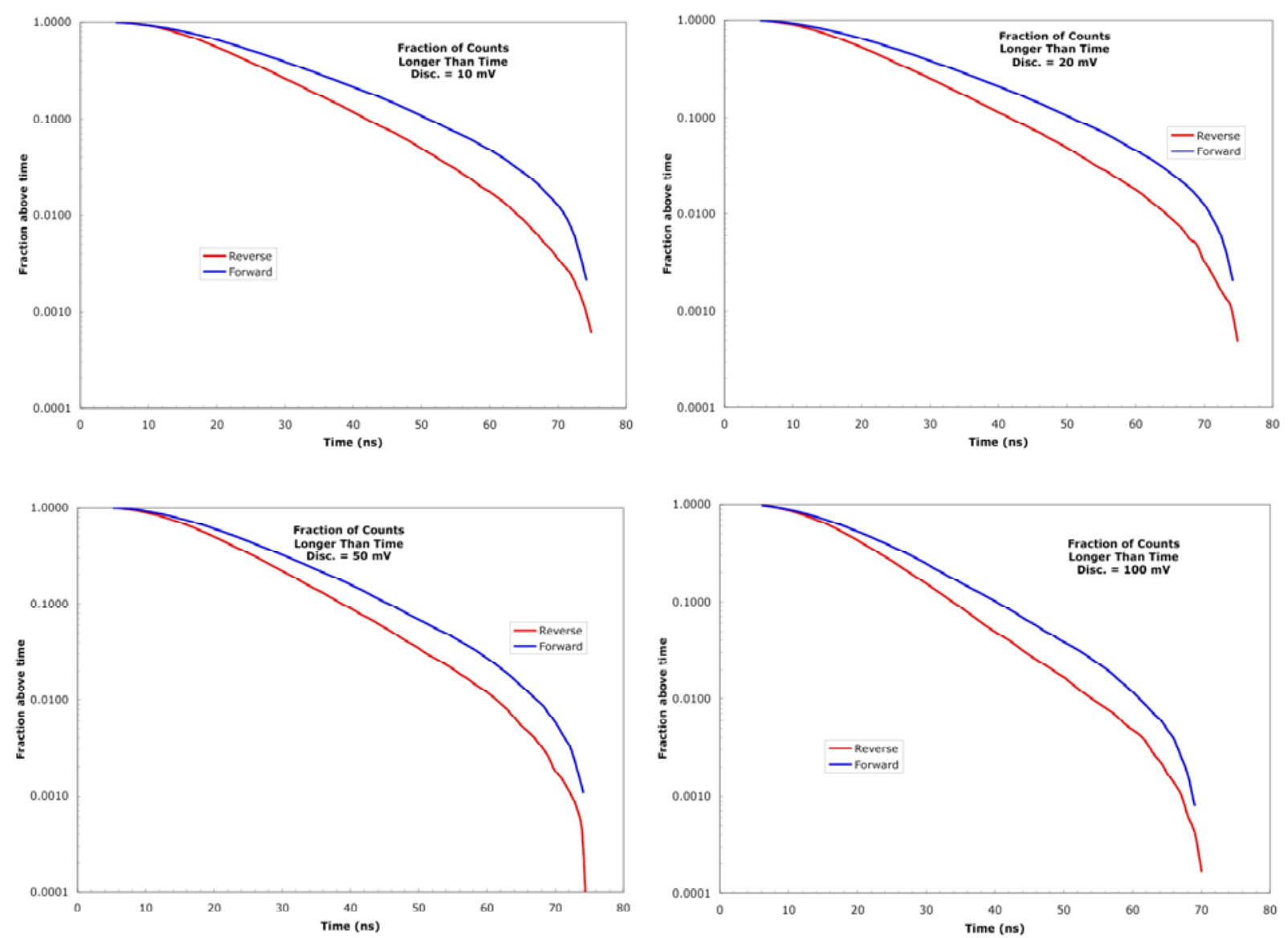

Figure 2.23. Fractional Efficiencies for Reverse and Forward Neutron TOF Spectra for Four Discriminator Levels
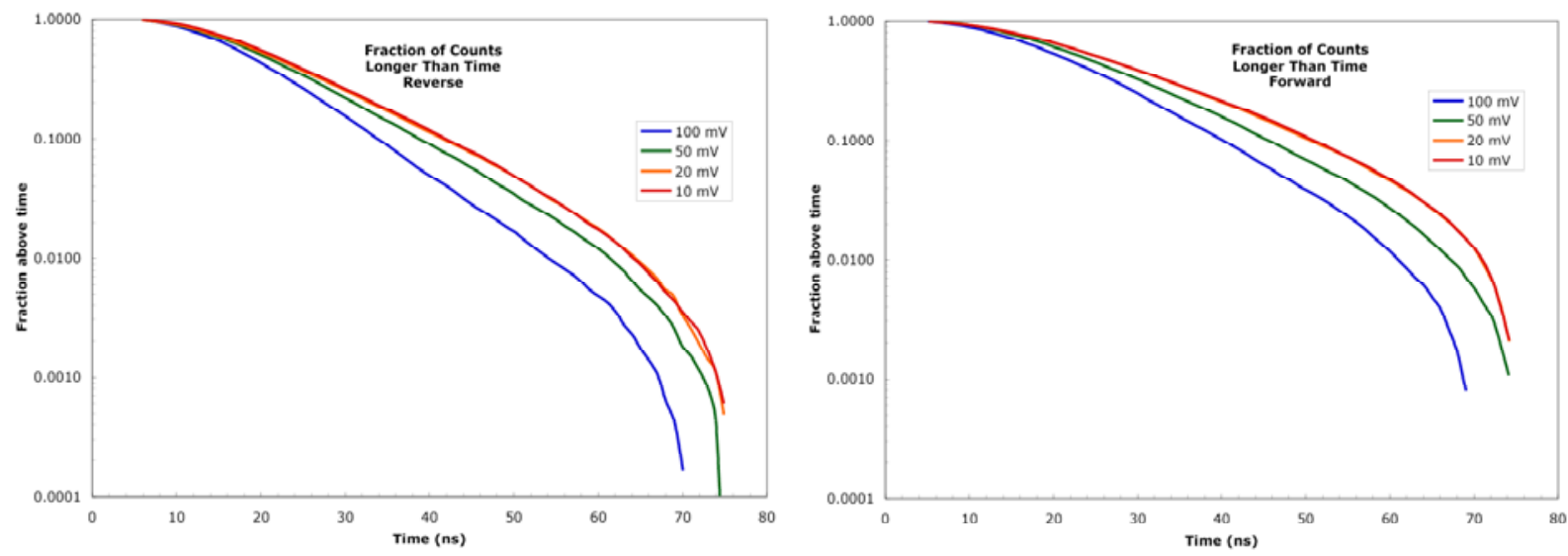

Figure 2.24. Comparison of Fractional Efficiency for ${ }^{252} \mathrm{Cf}$ in Reverse or Forward Distributions as a Function of Discriminator Levels 

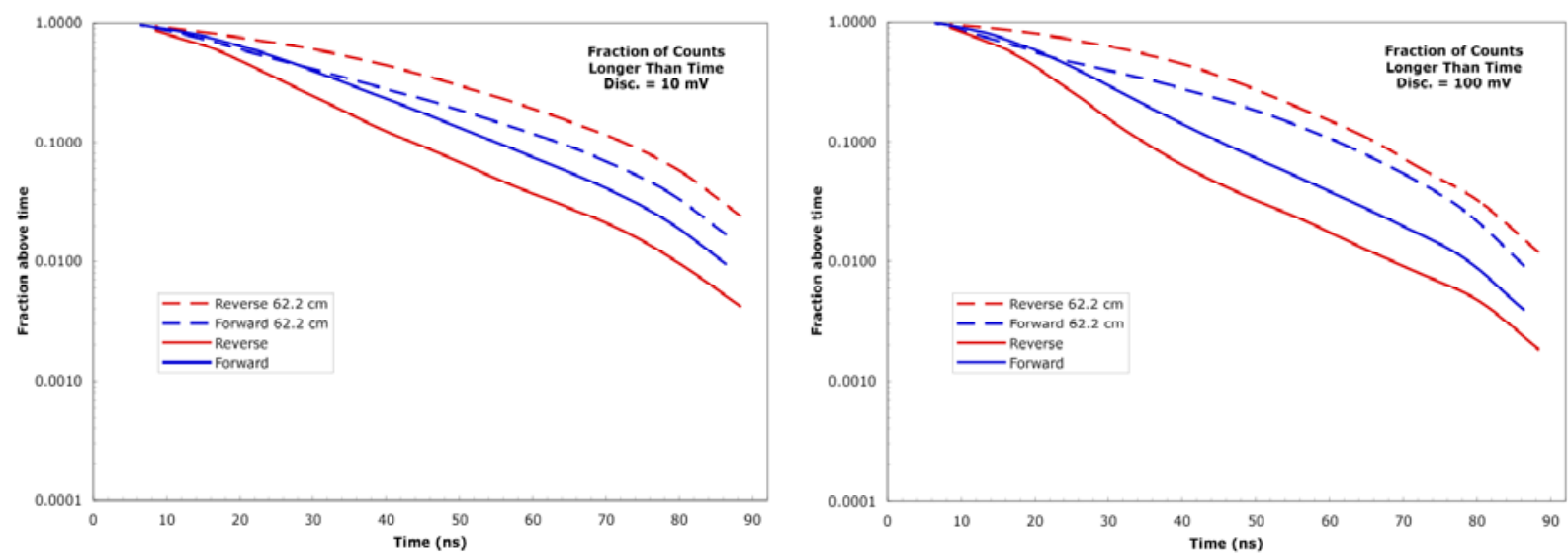

Figure 2.25. Fractional Efficiencies for ${ }^{252} \mathrm{Cf}$ at the Standard Source Distance and at a Distance 62.2-cm Farther Away

Figure 2.26 shows the relative efficiency as a function of discriminator for the Reverse, Forward, and Total neutron distributions.

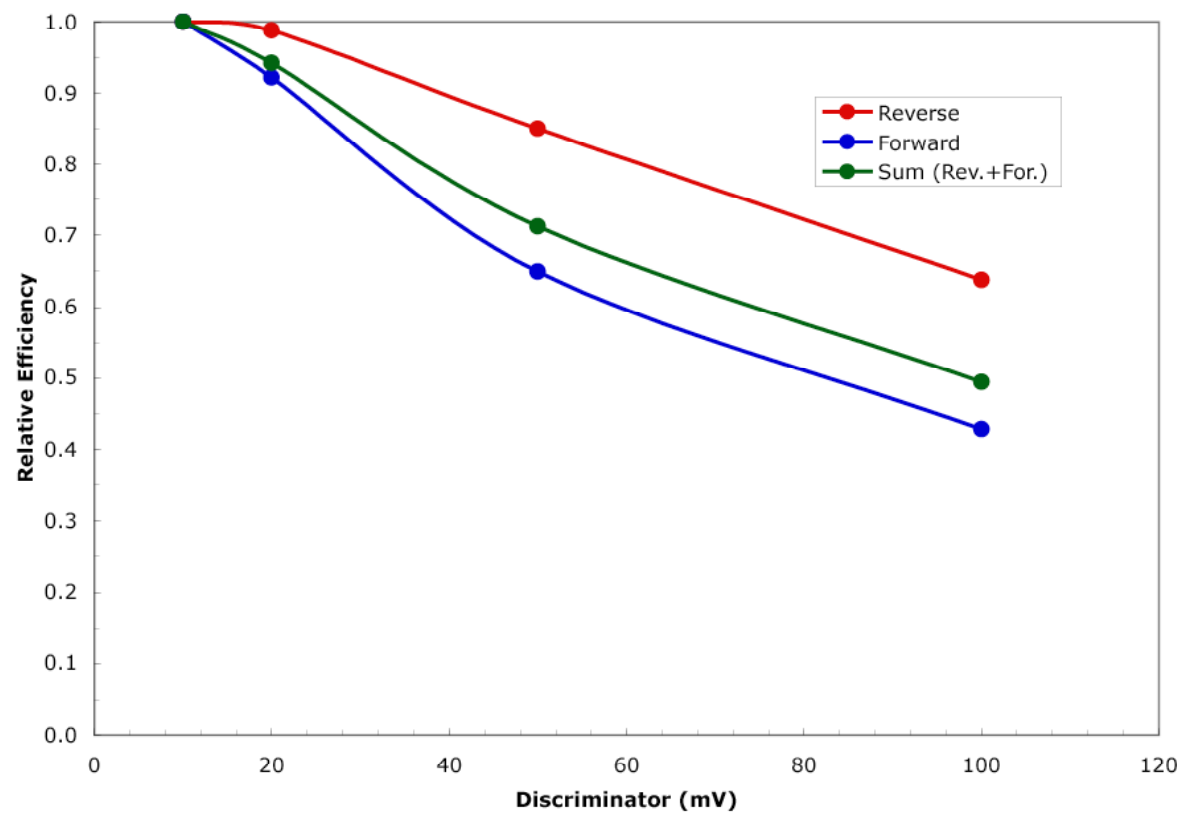

Figure 2.26. Relative Efficiency as a Function of Discriminator for Neutron TOF Spectra. The source is in the standard location.

\subsubsection{Comparison of Neutron and Gamma Fractional Efficiencies}

Figure 2.27 compares the fractional gamma and neutron efficiencies at the same discriminator level. These data can be used to quickly determine the relative effect of a given time threshold on the gamma and neutron efficiencies. 

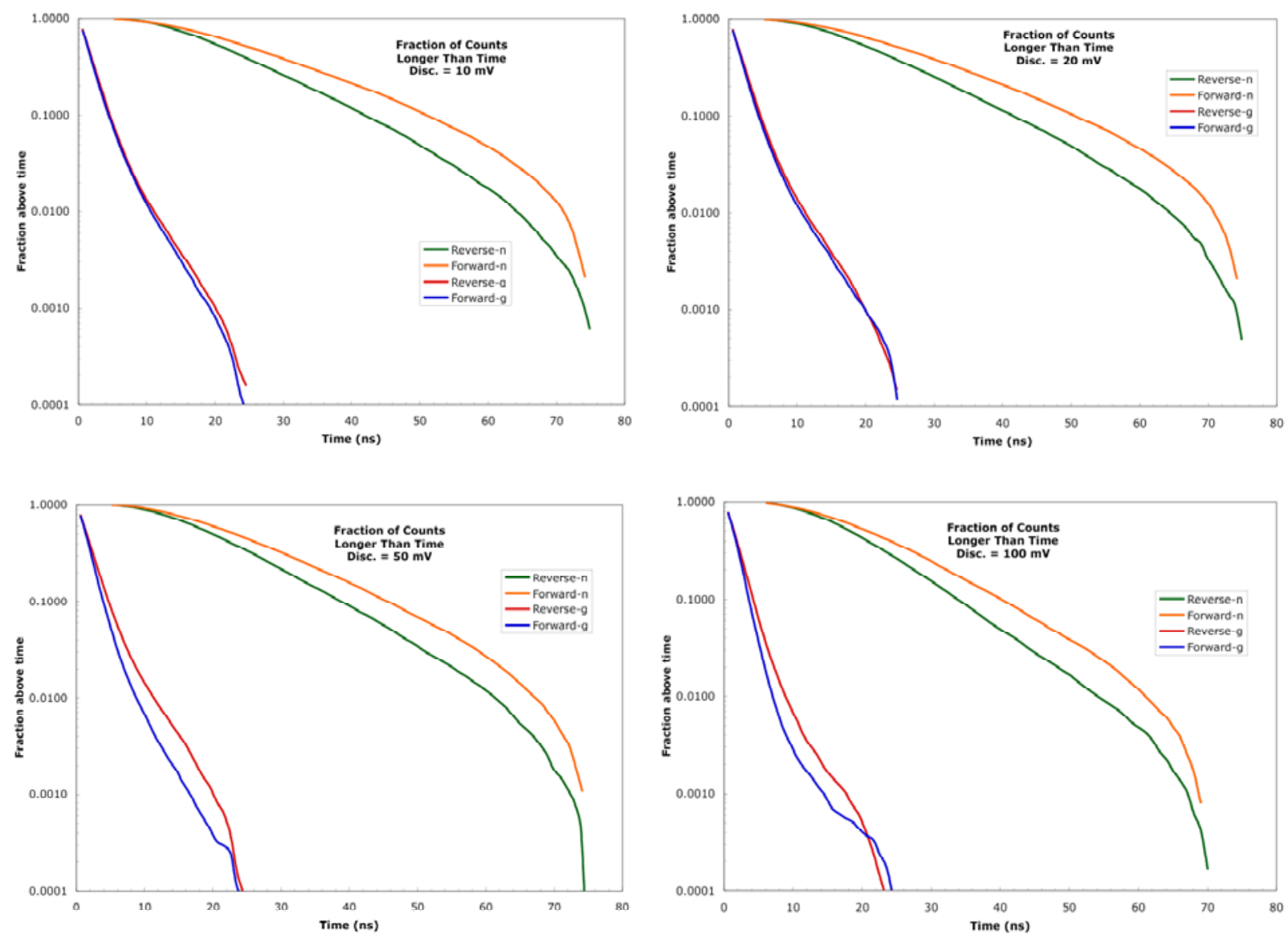

Figure 2.27. Neutron and Gamma Fractional Efficiencies. Sources are at standard location.

\subsubsection{Absolute Efficiencies}

The fractional efficiencies as a function of time threshold were converted to absolute efficiencies by correcting the fractional efficiencies by the source strength, the solid angle, and the differences between Forward and Reverse distributions. The source strength for the ${ }^{137} \mathrm{Cs}$ source is based on the original National Institute for Standards and Technology (NIST) calibration after correcting for decay from the date of calibration. The neutron source strength is based on a calibration done in April 1999 of a set of 26 identical sources. ${ }^{\text {(a) }}$ Only two of these sources were used here, so the neutron emission rate was reduced to the appropriate number of sources and corrected for decay from the date of calibration. Based on the original isotopic abundances of the $\mathrm{Cf}$ isotopes, a correction was calculated for the present abundance of ${ }^{250} \mathrm{Cf}$. This correction increased the source strength by $1 \%$. The difference in total counts in the Forward and Reverse distributions was not included in the fractional efficiencies calculated above, so it was necessary to make that correction here.

\subsubsection{Gamma}

As mentioned above, no intrinsic difference between the Forward and Reverse absolute efficiency is expected, and the total gamma efficiency should be based on the sum of the entire peak.

(a) $3.36 \times 10^{5}$ neutrons/second; David Stromswold (private communication). 
Figure 2.28 shows absolute efficiencies as a function of the time threshold for each of the discriminator settings. For the $10-\mathrm{mV}$ and $20-\mathrm{mV}$ discriminator levels, the Forward and Reverse absolute efficiencies are about the same. However, at the $50-\mathrm{mV}$ and $100-\mathrm{mV}$ discriminator levels, the Forward absolute efficiencies are significantly lower.
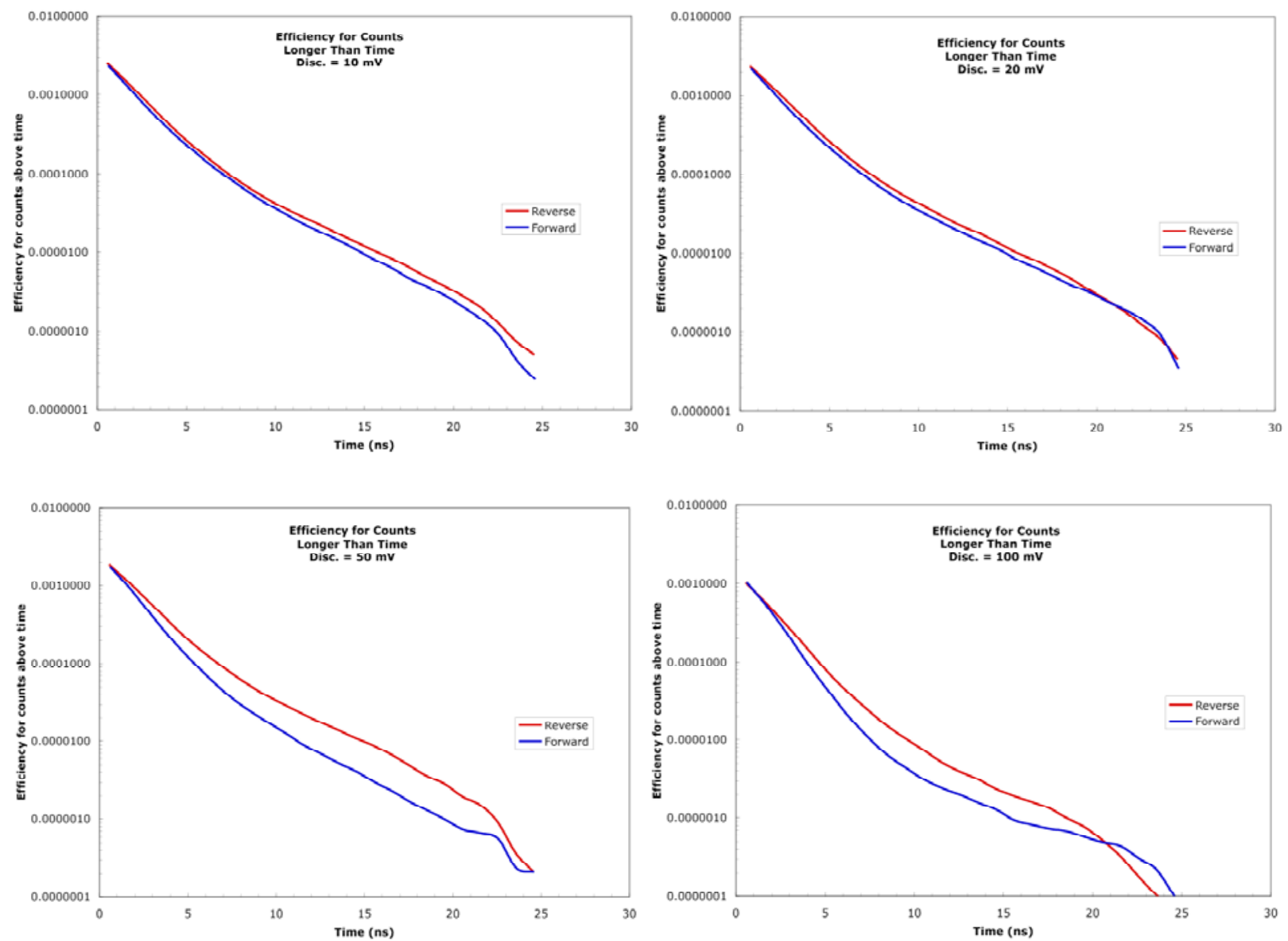

Figure 2.28. Absolute Efficiencies of the Gamma TOF Spectra as a Function of Time Threshold at each of the Four Discriminator Levels

Figure 2.29 shows absolute efficiencies for the Reverse and Forward distributions for all four discriminator levels. The trends to lower efficiency at higher discriminator are obvious. 

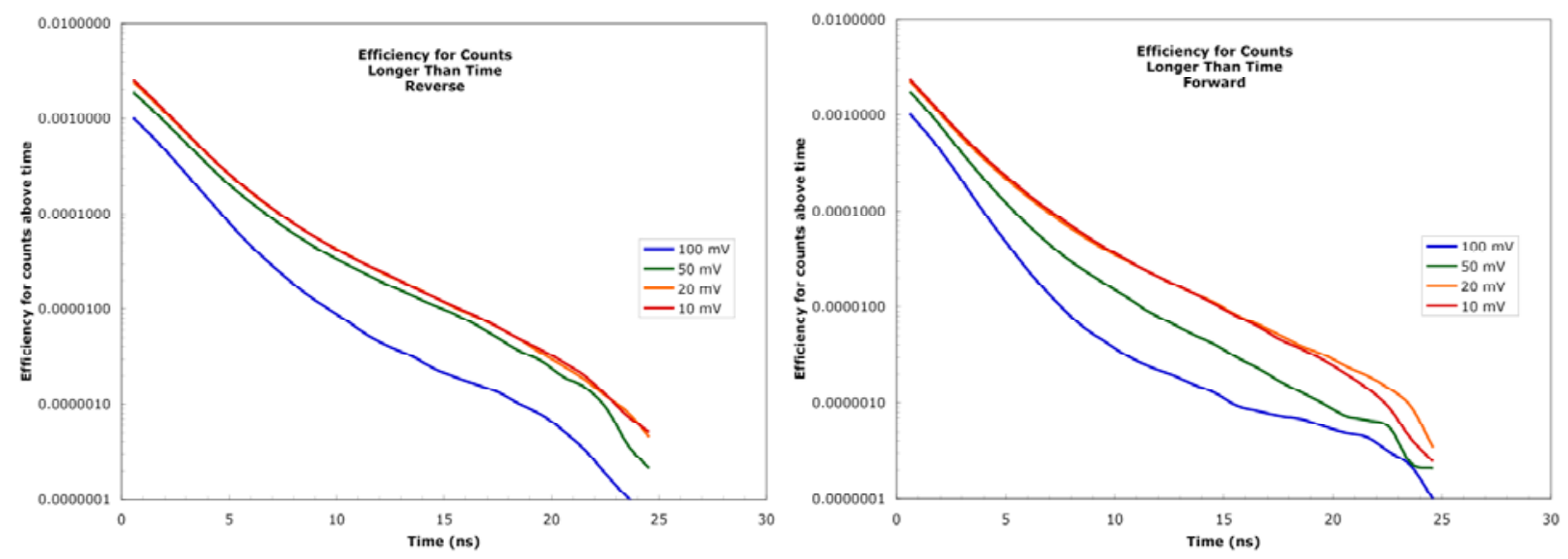

Figure 2.29. Absolute Efficiencies for the Reverse and Forward Gamma TOF Spectra for all four Discriminator Levels

Absolute efficiencies at two different source distances are shown for the discriminator level at 10 and $100 \mathrm{mV}$ in Figure 2.30. The good agreement in the absolute efficiency curves between the data at different distances indicates that the solid angle correction is reasonably accurate.
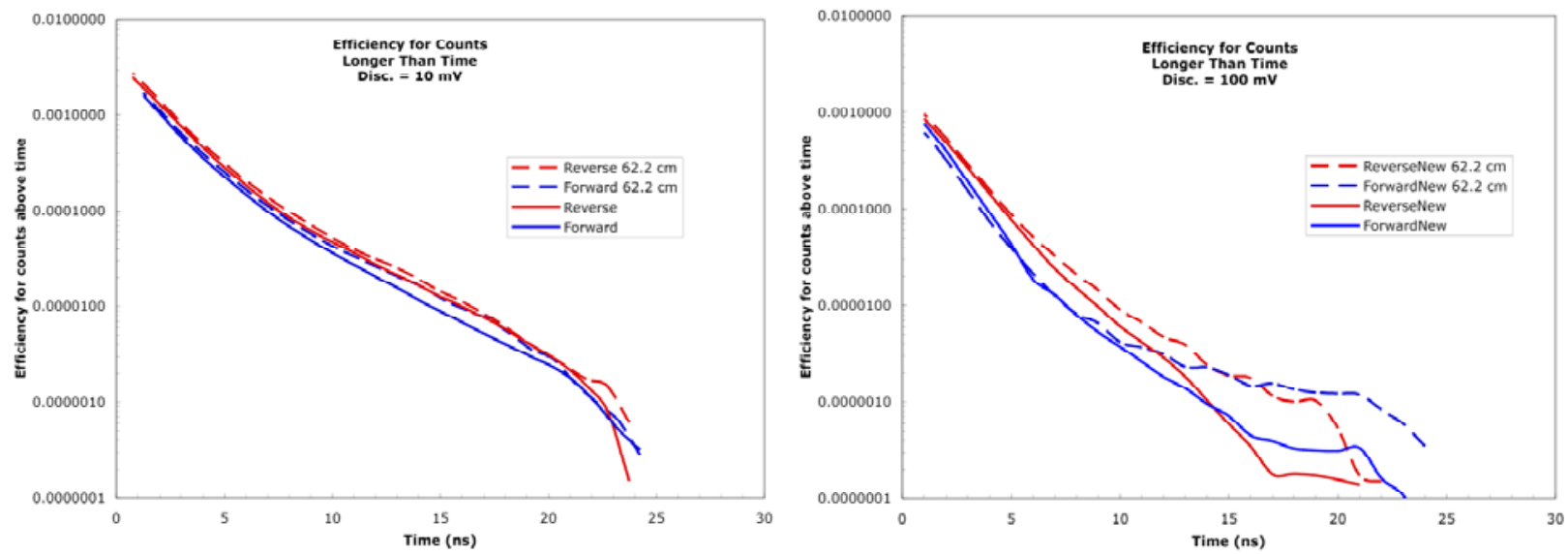

Figure 2.30. Absolute Efficiencies for Gamma TOF Spectra at the Standard Source Location and a Location 62.2-cm Farther Away

Figure 2.31 shows the absolute efficiency of total counts as a function of discriminator for the Reverse, Forward, and Total gamma distributions. Note that the maximum gamma efficiency is about $0.62 \%$ for the Sum of the entire gamma peak. Also note that the effect of the solid angle has been removed so that these efficiencies represent the efficiency for gammas striking the Front scintillator. 


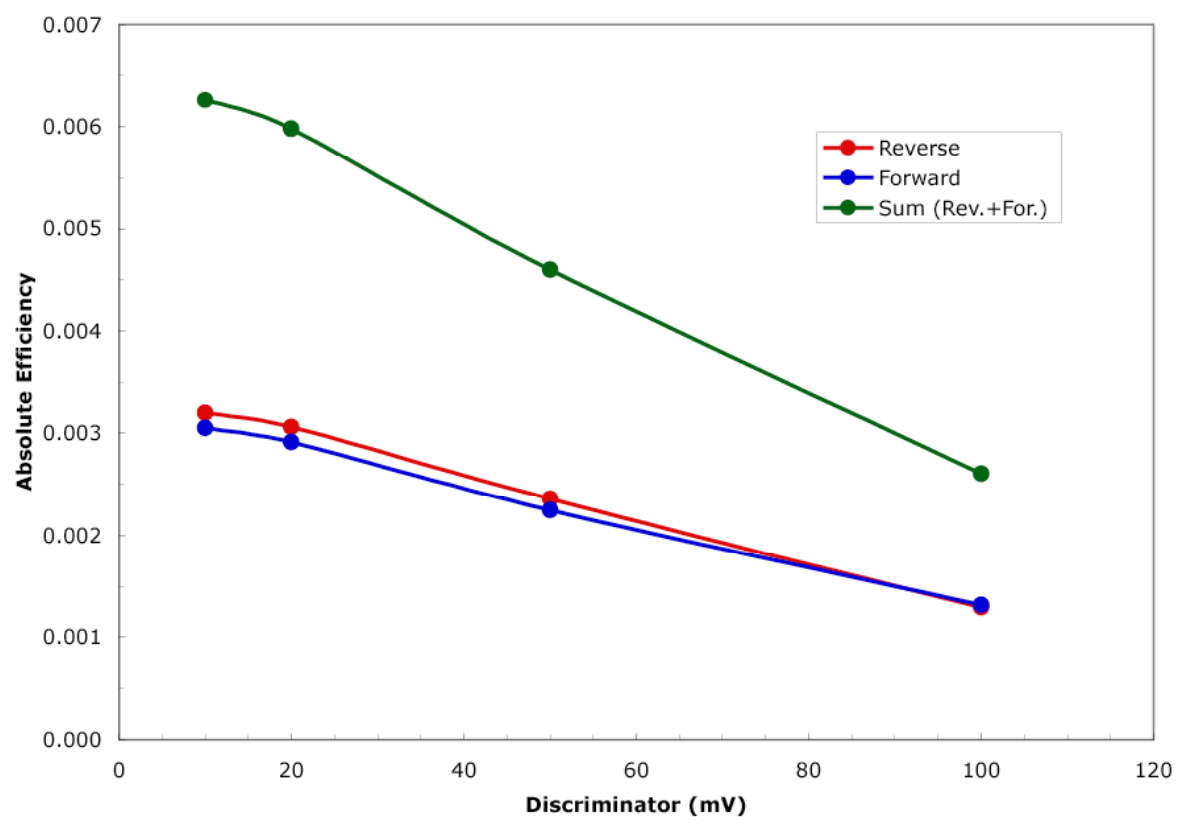

Figure 2.31. The absolute efficiency of total counts as a function of discriminator level for the Reverse, Forward, and Total gamma TOF distributions.

As mentioned in Section 2.2.1.2, the shape of the TOF spectra depends very little on the energy of the incident gamma. The detailed dependence of fractional efficiencies for ${ }^{137} \mathrm{Cs}$ as a function of the time threshold given above in Section 2.2.2.1 is assumed to be valid for other gamma sources. However, the absolute efficiencies as a function of time threshold must be corrected for the absolute efficiency of each source. Figures 2.32 and 2.33 show absolute efficiencies for ${ }^{137} \mathrm{Cs},{ }^{54} \mathrm{Mn}$, and ${ }^{60} \mathrm{Co}$. Note that the absolute efficiencies at the two different locations are quite similar because the solid angle correction from the source to the front face of the scintillator has been removed.

\subsubsection{Neutron}

The Forward and Reverse absolute efficiencies are based on different scattering processes. The Reverse distribution is predominantly caused by the presence of the lead shield, although a small portion of this response may arise from events in which the neutron suffers its first scattering the Back scintillator. The total neutron efficiency is the sum of the Forward and Reverse efficiencies if a lead shield is used. If no lead shield is used, the sensor's neutron response will be dominated by the Forward efficiency contribution.

Figure 2.34 shows the absolute efficiencies for neutrons as a function of time threshold. In every case, the Reverse distribution decreases more rapidly than the Forward distribution, indicating different spectral shapes. 


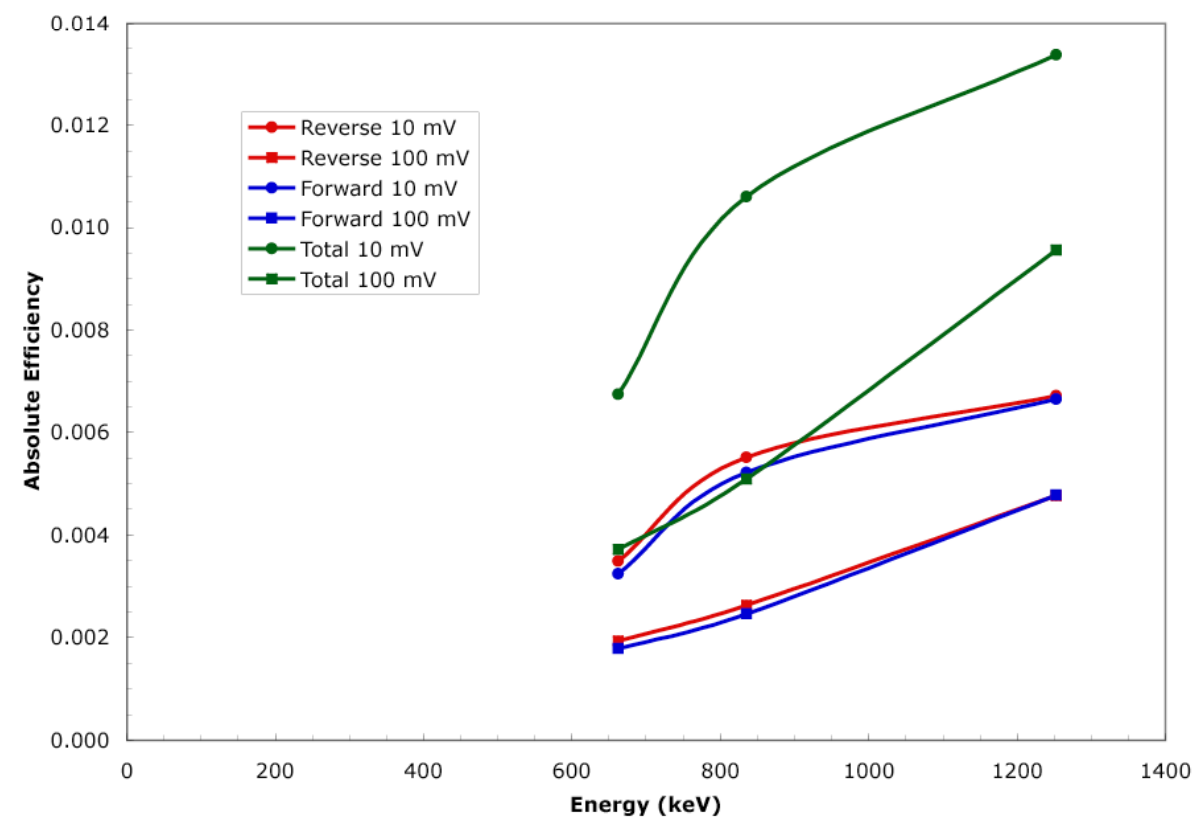

Figure 2.32. Absolute Efficiency as a Function of Incident Gamma Energy for Sources at the Normal Location

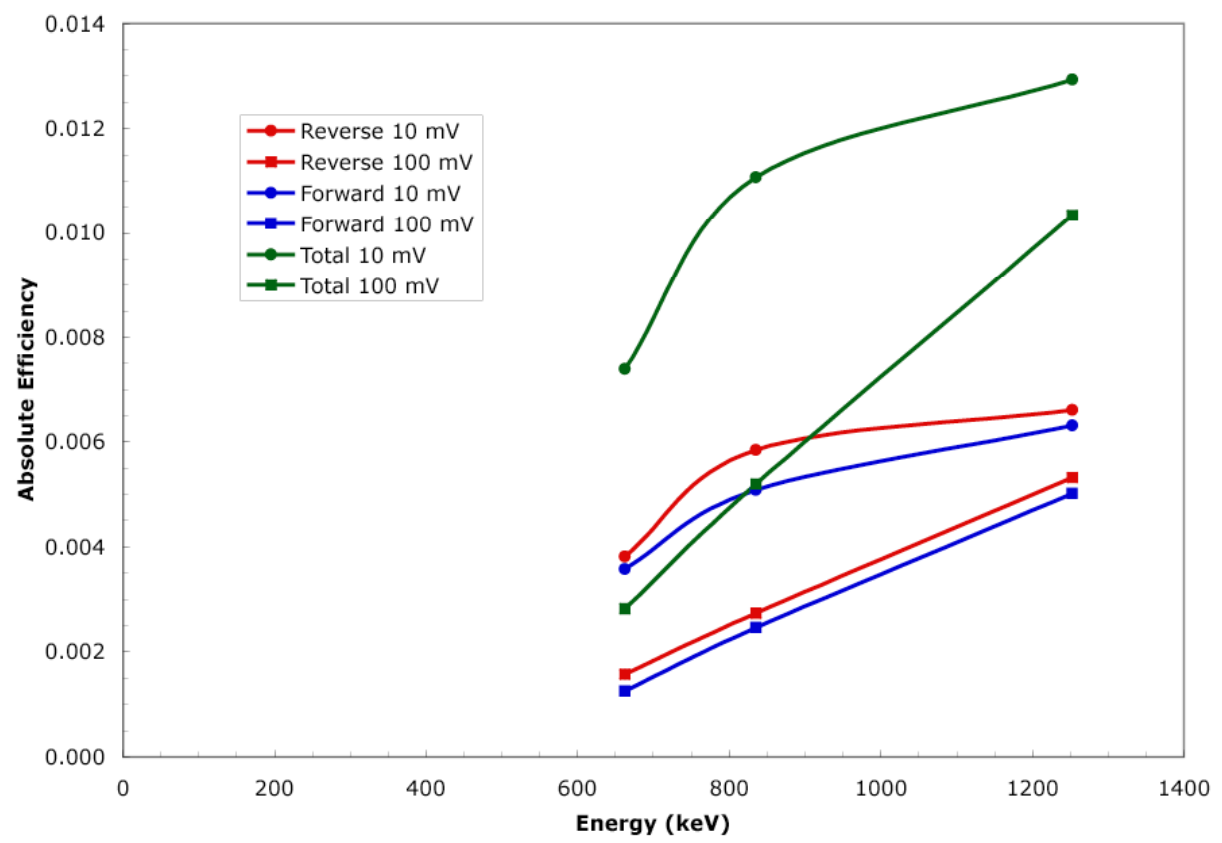

Figure 2.33. Absolute Efficiency as a Function of Gamma Energy for Sources Located $62.2 \mathrm{~cm}$ from the Normal Position 

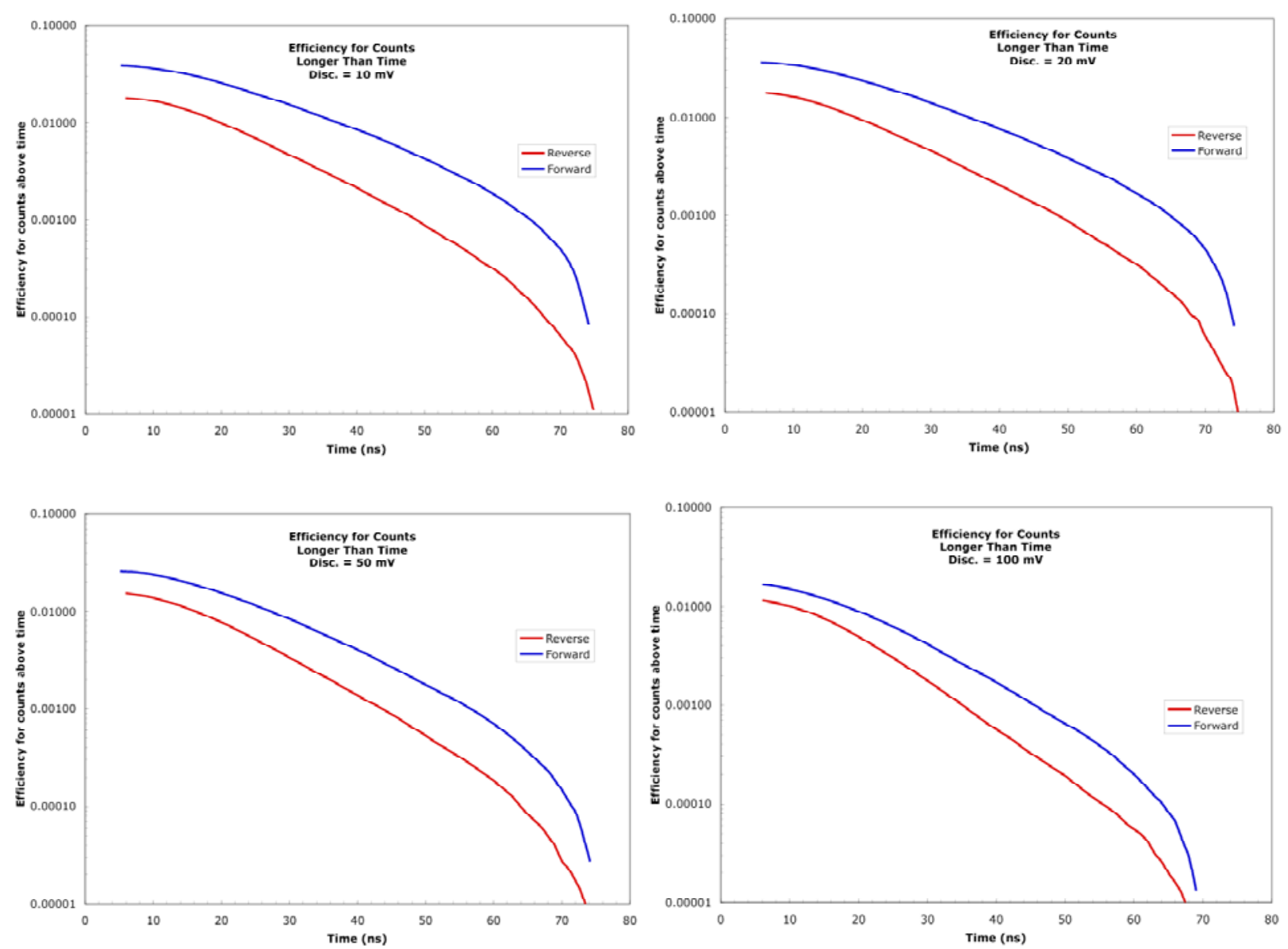

Figure 2.34. Absolute Efficiencies for Neutrons as a Function of Time Threshold

Figure 2.35 shows absolute efficiencies for the Reverse and Forward distributions for all four discriminator levels. The decrease in efficiency as the discriminator increases is clear. The narrowing of the distribution indicates an increase in the detected rate of higher energy neutrons relative to lower energy neutrons; i.e., fewer events at long time intervals mean fewer low-energy neutrons.

Absolute efficiencies as a function of time threshold at different source distances are shown for two discriminator levels in Figure 2.36. The spectral shapes disagree for the two distances, and it appears that the efficiency for back-scattered neutrons (Reverse) is reduced more than the Forward at the longer distance. 

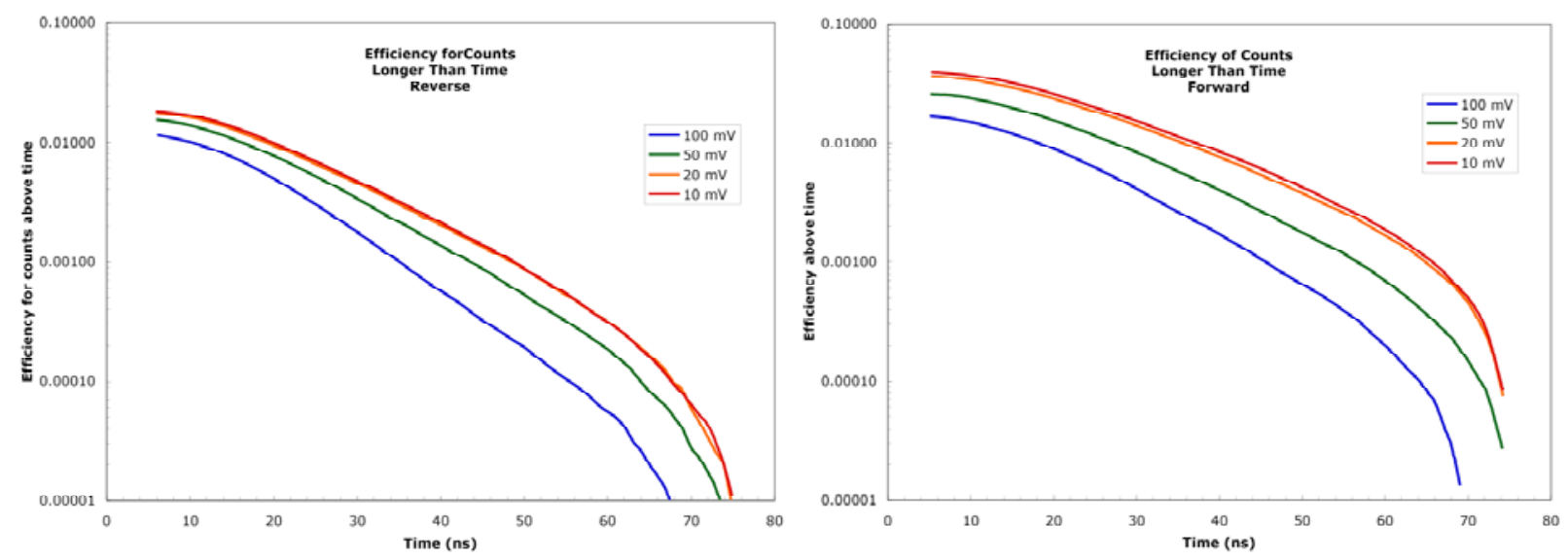

Figure 2.35. Neutron Absolute Efficiencies for the Reverse and Forward Distributions
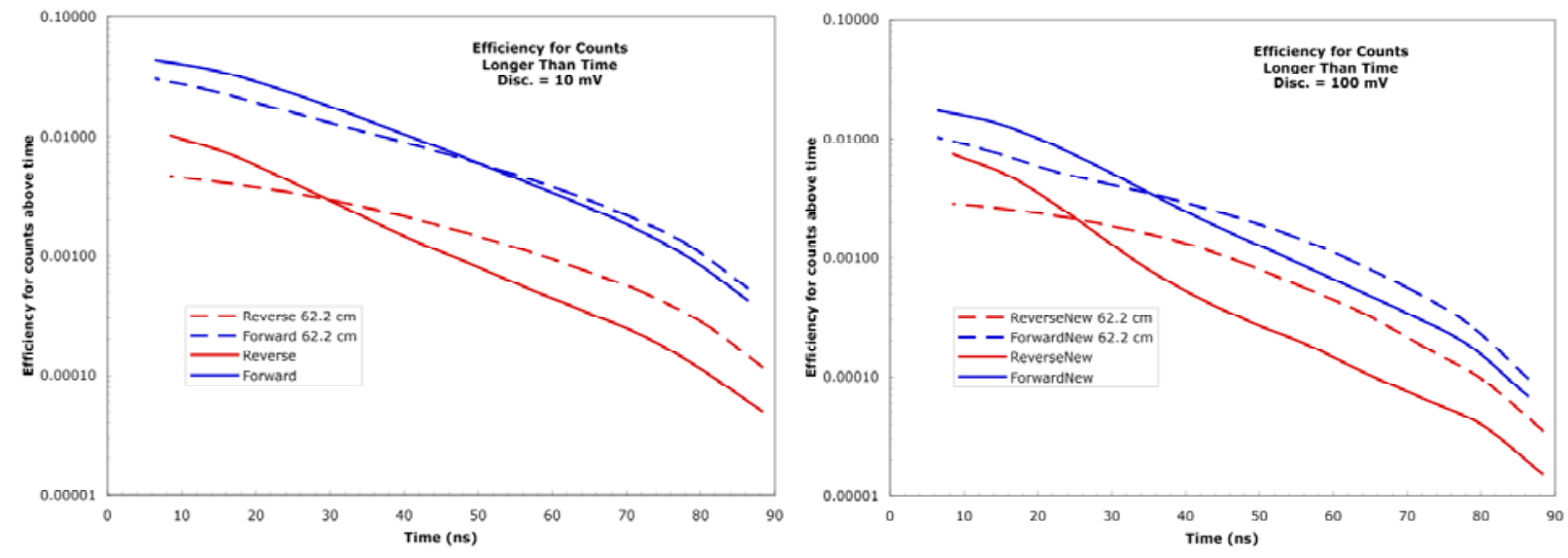

Figure 2.36. Neutron Absolute Efficiencies as a Function of Time Threshold at the Standard Source Location and a Location 62.2-cm Farther away

Figure 2.37 shows the absolute efficiency of total counts as a function of discriminator for the Reverse, Forward, and Total neutron distributions. Note that the maximum neutron efficiency is about $6 \%$ for the sum of the Forward and Reverse spectra. The Forward efficiency is more sensitive to the discriminator than the Reverse. 


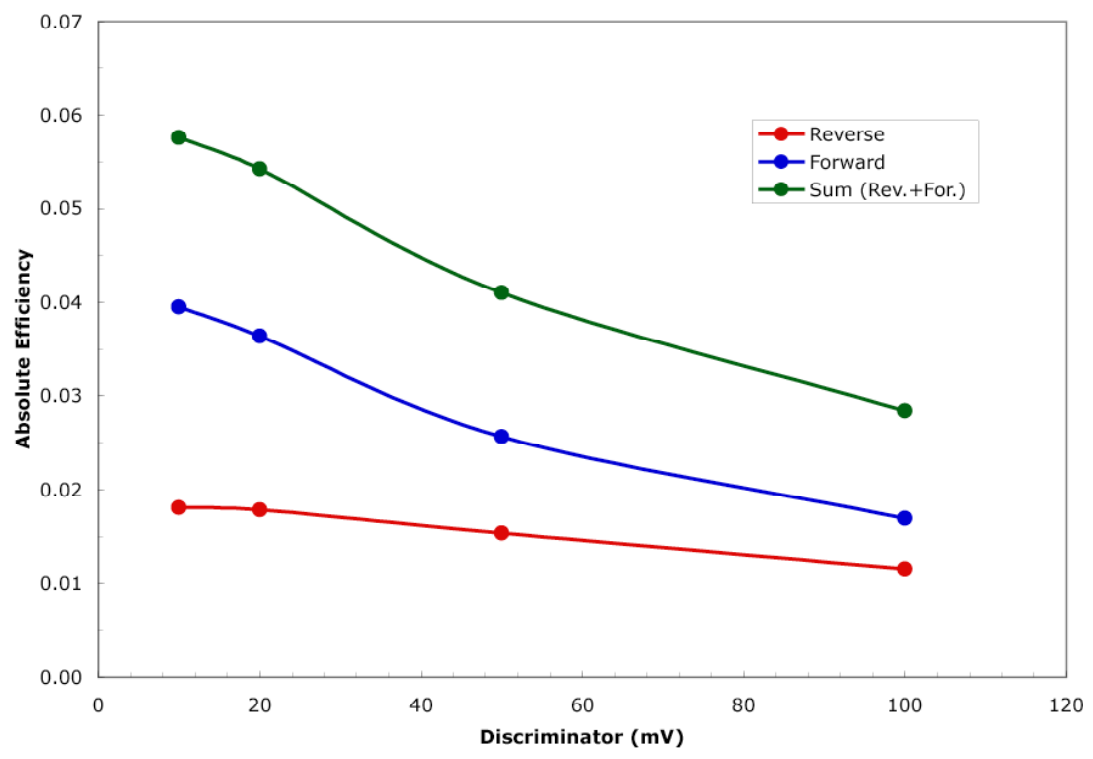

Figure 2.37. Absolute Efficiency of Total Counts in Neutron TOF Spectra as a Function of Discriminator Level

\subsubsection{Comparison of Neutron and Gamma Absolute Efficiencies}

The absolute gamma and neutron efficiencies at the same discriminator level are compared in Figure 2.38 for ${ }^{137} \mathrm{Cs}$ gammas and ${ }^{252} \mathrm{Cf}$ neutrons. These data can be used to quickly determine the gamma contamination at a given time threshold. Note that the gamma efficiencies for sources with higher gamma energy should be increased according to the trends shown in Figure 2.32 and 2.33.

\subsection{Determination of Neutron Energy Spectra}

In most neutron TOF detector systems, the flight path between the neutron detectors is much larger than the dimensions of the scintillator detectors. Thus, the uncertainties in path length caused by the thickness of the detectors or the location of the event within the detectors are small compared to the distance between the detectors. In such systems, the TOF can be converted to a velocity or energy spectrum.

The dimensions of the TOF system tested here, and the limited resolution in each scintillator's interaction position as derived from timing information in the "double-ended" PMT configuration, preclude accurate determination of the particle flight path. Thus only a crude measurement of the incident neutron energy spectrum based upon the TOF (with or without a correction to the flight path based upon the measured interaction position in each scintillator) is possible with the present system, and this crude energy does not enhance the system's capability to distinguish gammas from neutrons. However, the ALPS II detector was designed for optimizing the resolution of the pulse-height spectrum in each scintillator. With six PMTs on a single scintillator, we previously measured a resolution of about $25 \%$ full-width halfmaximum (FWHM) for the Compton edge peak from a ${ }^{137} \mathrm{Cs}$ source. The energy of the Compton edge for the ${ }^{137} \mathrm{Cs}$ gamma is $477 \mathrm{keV}$. This energy is comparable to the energies that might be deposited in each scintillator by scattering of a $1-\mathrm{MeV}$ neutron. On the assumption that a valid neutron TOF event deposits most of the incident neutron energy in the two scintillators, the sum of the pulse heights in each scintillator should be a useful measure of the neutron energy. The ALPS II detector system can thus be 
used to identify an event as a neutron based on the TOF measurements described here. Simultaneously, the energy of the neutron can be determined by the summed pulse heights.
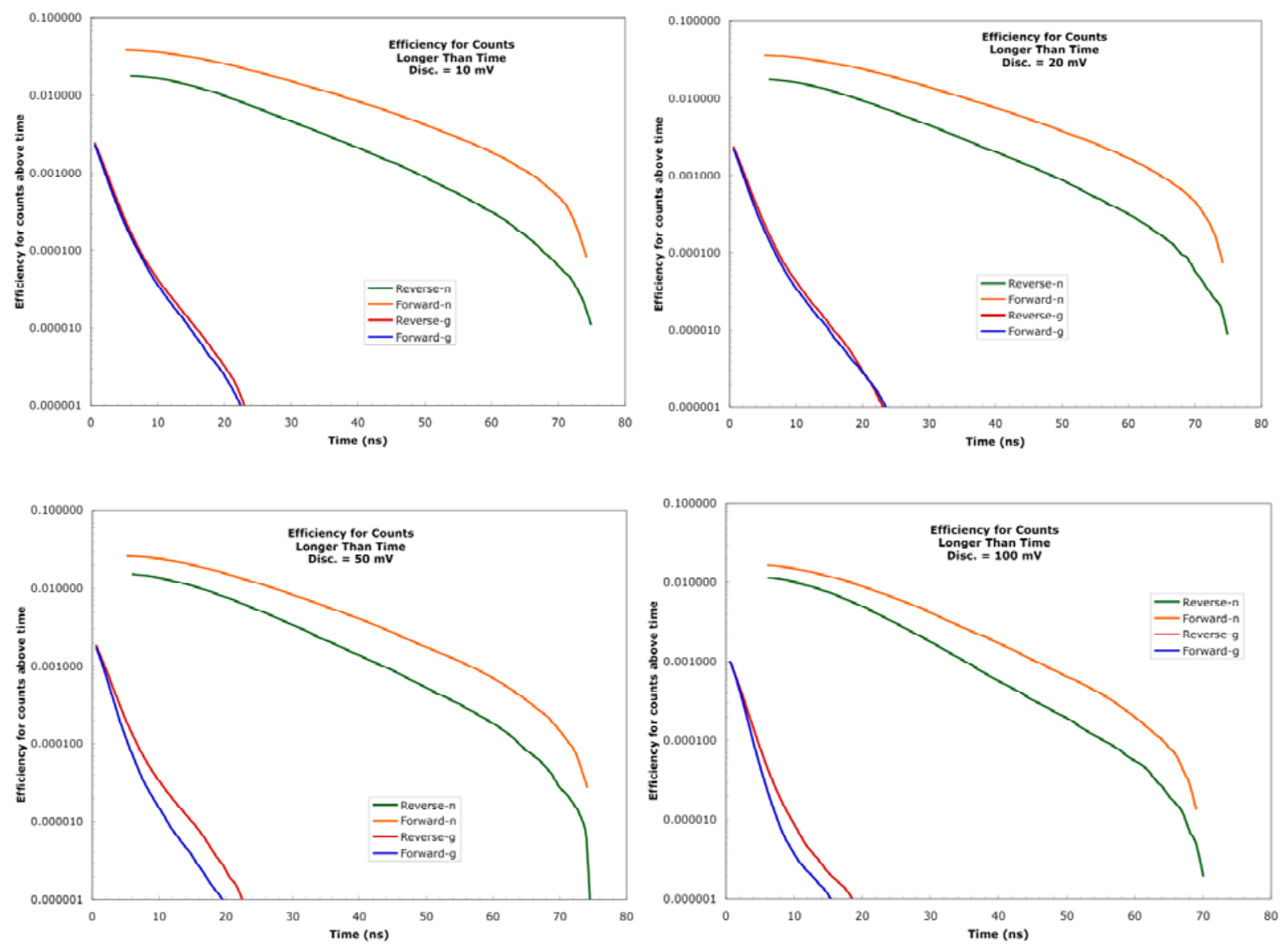

Figure 2.38. Comparison of Absolute Gamma and Neutron Efficiencies as a Function of Time Threshold

\subsubsection{Experiments}

Preliminary experiments to evaluate this concept were performed. The counting electronics shown in Figure 2.5 were modified by adding a multi-channel charge-to-digital converter (QDC). The signals from all six PMTs on the Front scintillator were fed to the QDC as well as the signals from PMTs 8 and 11 on the Back scintillator. An insufficient number of delay boxes prevented the measurement of the other four PMTs on the Back scintillator. The data-acquisition program was modified to record 21 raw and calculated parameters for each event. A number of single- and two-parameter histograms were calculated and displayed on-line. These plots included a pulse-height spectrum for the Front scintillator based on the average pulse-height spectrum of all six PMTs, a pulse-height spectrum for the Back scintillator based on the average pulse-height spectrum from 2 PMTs, a Summed pulse-height spectrum based on the sum of the average Front and Back pulse-height spectra, and plots of TOF versus pulse height of the Front, Back, and Summed. 


\subsubsection{Results}

Figure 2.39 shows the Summed pulse-height spectra for the ${ }^{137} \mathrm{Cs},{ }^{54} \mathrm{Mn}$, and ${ }^{60} \mathrm{Co}$ gamma sources. The pulse-height spectra did not give as good resolution as obtained previously, partly because of incomplete coverage of the Back scintillator by PMTs, but primarily because of electronic "ringing" from an unknown pulsed frequency source. This problem could not be solved in the time available for this experiment.

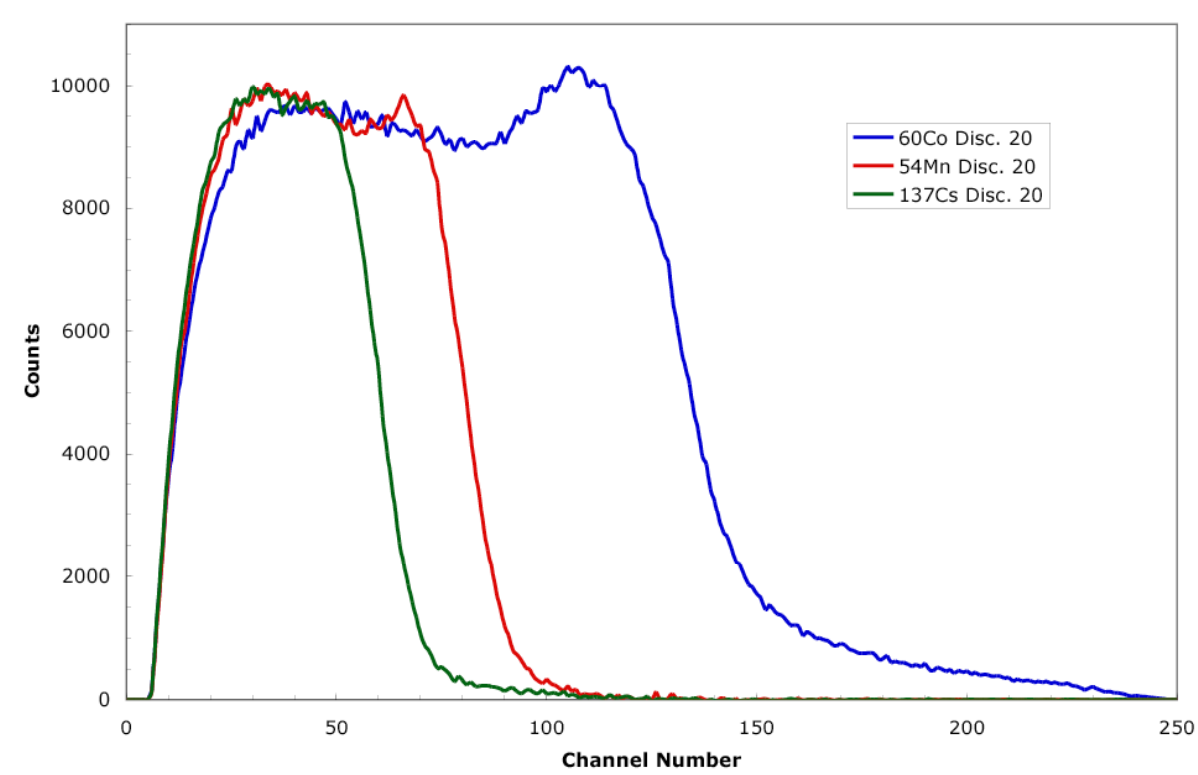

Figure 2.39. Pulse-Height Spectra for Three Gamma Sources Based on the Sum of the Pulse-Height Spectra of the Front and Back Scintillators

The Summed pulse-height spectra for the ${ }^{252} \mathrm{Cf}$ neutron source are shown in Figure 2.40 for two settings of the TOF discriminator levels. The small peak near channel 130 may be an electronic artifact as it is not consistent in the spectra at different discriminator levels.

Note that these spectra include both neutron and gamma events but are dominated by the gamma events. By plotting the TOF data versus the pulse-height data, we can separate the pulse-height spectra of the neutrons from that of the gammas. Figure 2.41 provides an example of the TOF vs Pulse Height with a gamma source. Figure 2.42 shows a similar example for the neutron source. Time zero as defined above for the TOF distributions is in channel 256 for these histograms. In both cases, the gamma events are in a narrow TOF region clustered around time zero. The ${ }^{252} \mathrm{Cf}$ source, however, has additional events broadly distributed on the TOF axis that are caused by the more slowly moving neutrons. 


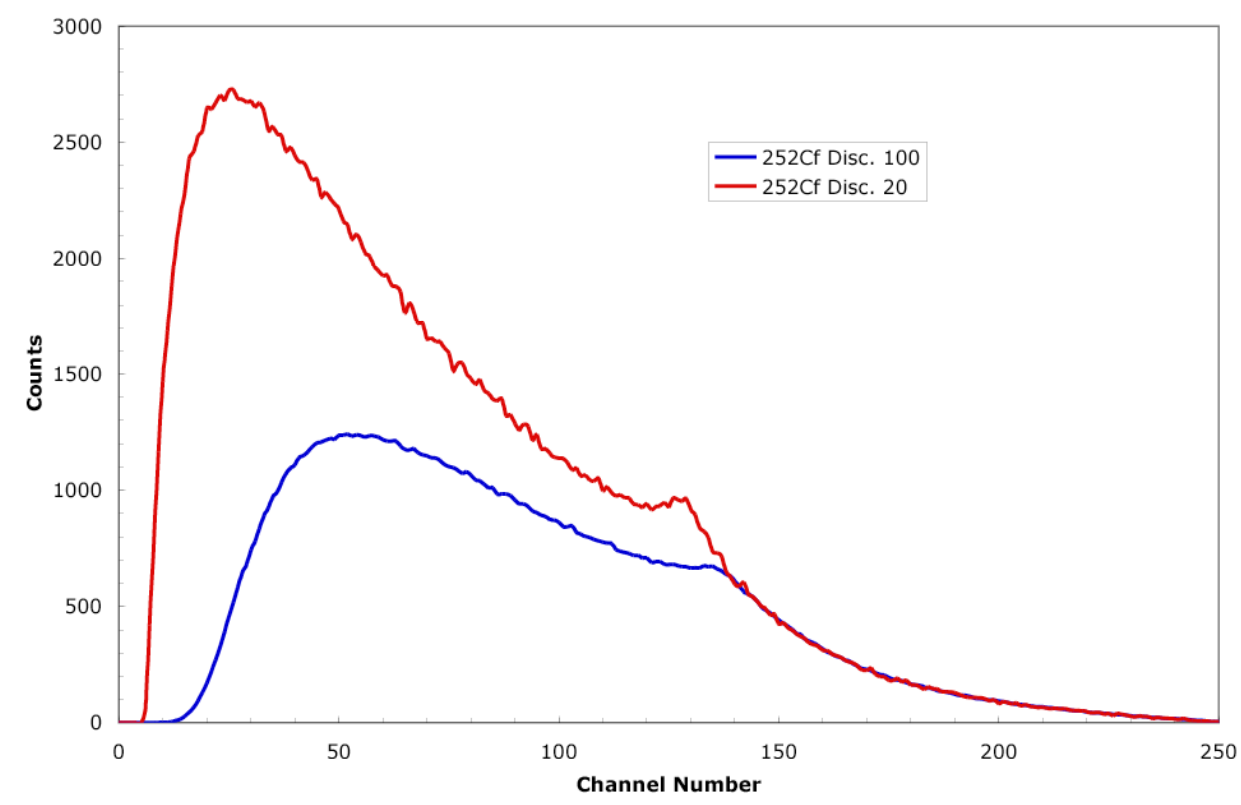

Figure 2.40. Pulse-Height Spectra for Two Discriminator Levels for the ${ }^{252}$ Cf Source Based on the Sum of the Pulse-Height Spectra of the Front and Back Scintillators

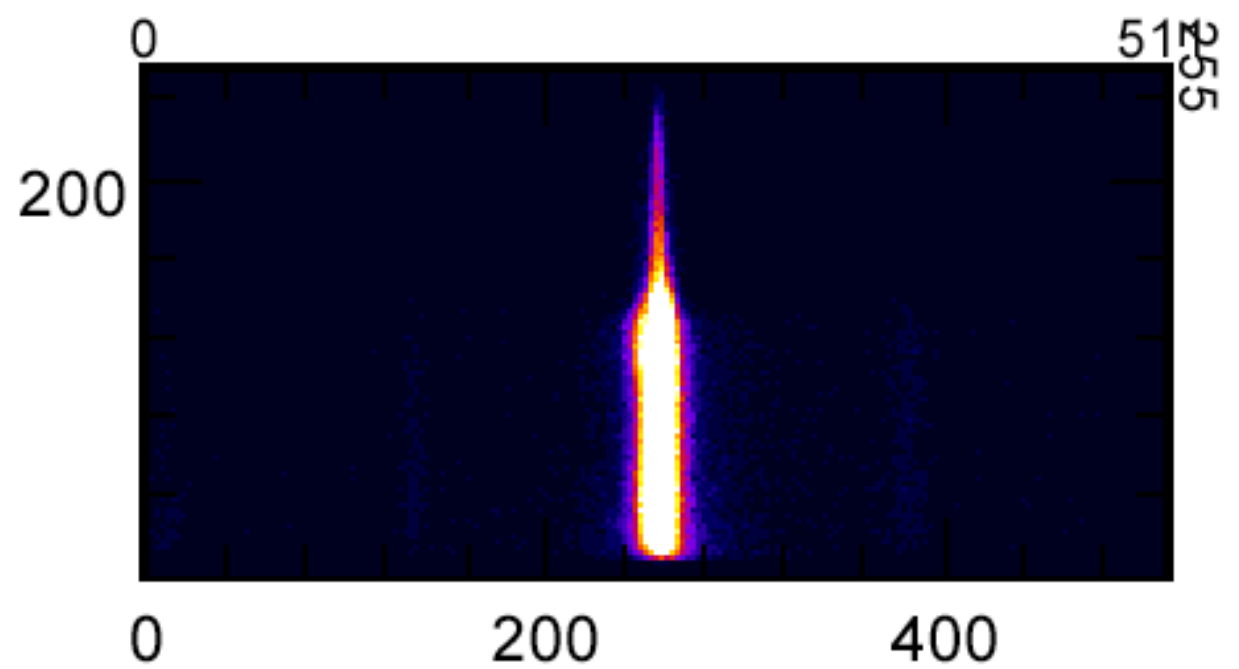

Figure 2.41. TOF (horizontal axis) Versus Summed Pulse Height (vertical axis) for the ${ }^{60} \mathrm{Co}$ Source with the TOF Discriminator at $20 \mathrm{mV}$ 


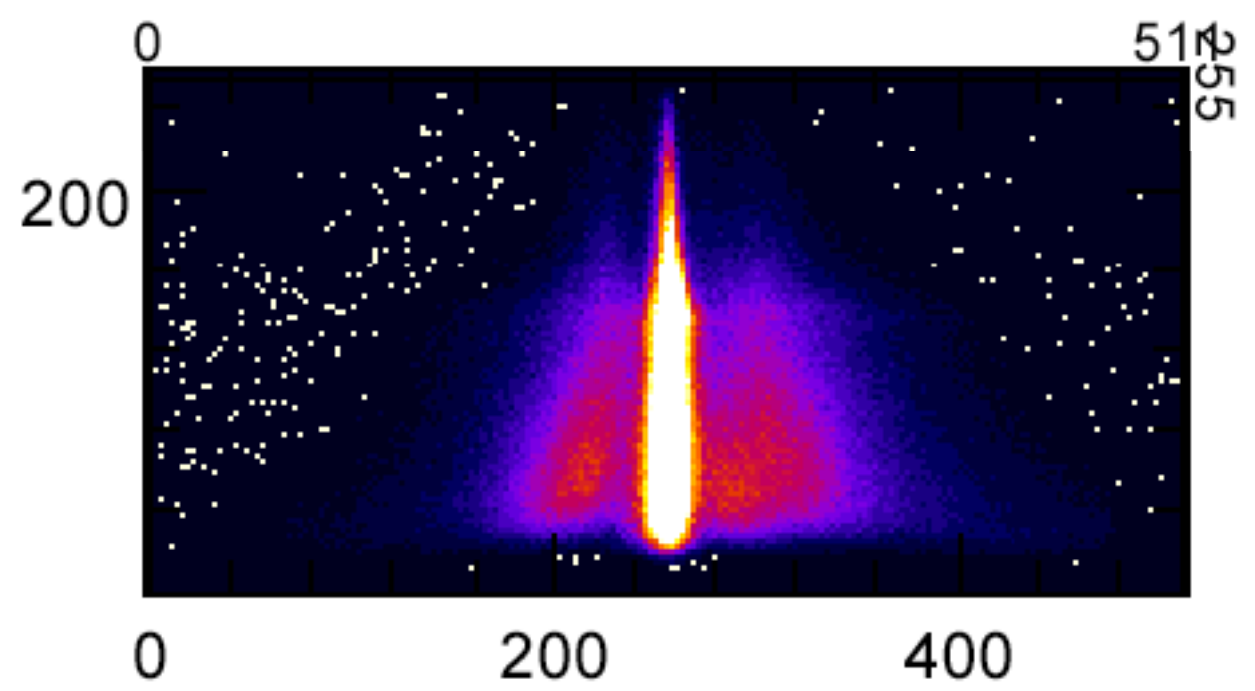

Figure 2.42. TOF (horizontal axis) versus Summed pulse height (vertical axis) for the ${ }^{252} \mathrm{Cf}$ source with the TOF discriminator at $100 \mathrm{mV}$

Setting a gate on the TOF axis to eliminate gamma events allows separation of the neutron pulse-height spectrum from the gamma pulse-height spectrum. For example, setting a gate from channel 10 to 243 on the TOF axis and collapsing the data to the pulse-height axis gives the pulse-height spectrum caused by neutrons with the Reverse mechanism, i.e., neutrons that reflect off the lead shield and interact in the Back scintillator before interacting in the Front scintillator. Setting a gate from channel 277 to 501 on the TOF axis gives the pulse-height spectrum due to neutrons with the Forward mechanism, i.e., neutrons that interact in the Front scintillator before interacting in the Back scintillator. Similarly, the gamma spectrum is obtained by setting the gate on the TOF axis from channel 244 to 276 . The pulse-height spectra for these different processes are compared to the total neutron plus gamma spectrum in Figure 2.43. In the case of ${ }^{252} \mathrm{Cf}$, the shape of the gamma pulse-height spectrum is very similar to the shape of the neutron spectra. From Figure 2.39, we know that the gamma pulse-height spectrum depends on the gamma source. Presumably, the shape of the neutron spectra will also be uniquely characteristic for different neutron sources. This technique should thus be capable of identifying different neutron sources, even in the presence of other gamma sources. ${ }^{\mathrm{a}}$

\footnotetext{
${ }^{a}$ The detailed shape of the neutron TOF distribution will also presumably correlate to the incident neutron energy spectrum. The extent to which the pulse height information described here supplements the inherent capability of the TOF system to distinguish neutron sources could not be quantified in this project's experimental campaign, because measurements with only a single neutron source type $\left({ }^{252} \mathrm{Cf}\right)$ were permitted in the facility housing the sensor.
} 


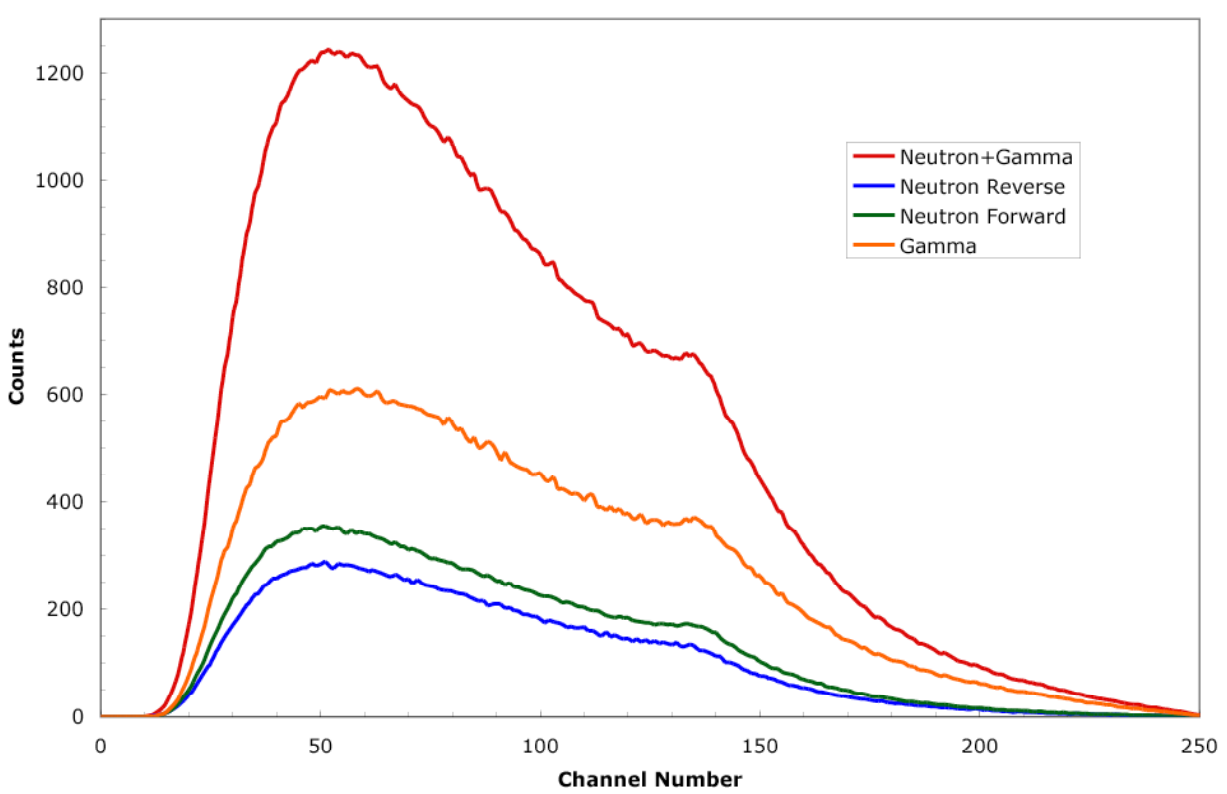

Figure 2.43. Total Pulse-Height Spectra for the ${ }^{252} \mathrm{Cf}$ Source for Different Processes Resulting from Gates set on the TOF Distribution

Pulse-height spectra in the Front scintillator versus TOF and pulse-height spectra in the Back scintillator versus TOF can be analyzed to give pulse-height spectra for specific mechanisms in the same manner as the Total pulse-height spectra shown in Figure 2.43. The resulting spectra have no sharp features, but the shapes of the spectra depend on the type of interaction. A weighted average channel number was calculated for each spectrum. This channel number is proportional to the average energy deposited in each scintillator for the various interactions. The results are listed in Table 2.1, which gives the average channel numbers for the Front, Back, and Total scintillators for the Forward Neutron, Reverse Neutron, and Gamma interactions.

Table 2.1. Weighted Average Channel Numbers for Pulse-Height Spectra Derived from Two-Dimensional Plots of Pulse-Height Spectra Versus TOF

\begin{tabular}{|c|c|c|c|}
\hline & Forward Neutron $^{(a)}$ & Reverse Neutron $^{(b)}$ & Gamma $^{(c)}$ \\
\hline Front Scintillator & 101.3 & 74.8 & 99.7 \\
\hline Back Scintillator & 70.0 & 95.8 & 87.4 \\
\hline Total $^{(\mathrm{d})}$ & 85.7 & 85.4 & 93.5 \\
\hline \multicolumn{4}{|c|}{$\begin{array}{l}\text { (a) Sum of channels } 277 \text { to } 501 \text { on TOF axis. } \\
\text { (b) Sum of channels } 10 \text { to } 243 \text { on TOF axis. } \\
\text { (c) Sum of channels } 244 \text { to } 276 \text { on TOF axis. } \\
\text { (d) Pulse-height values are average of pulse heights in Front and Back scintillators. }\end{array}$} \\
\hline
\end{tabular}

The data in Table 2.1 show that when the neutrons interact first in the Front scintillator, they tend to deposit more energy in the Front scintillator than the Back scintillator. When the neutrons interact first in the Back scintillator, they tend to deposit more energy in the Back scintillator than the Front scintillator. These results are consistent with the fact that the first neutron scattering event necessarily involves a higher energy neutron than the second scattering event. The data also indicate that when the pulse heights 
in the Front and Back scintillators are combined, the resulting Total energy is the same regardless of the sequence of scattering events.

An unexpected result of plotting the TOF versus the pulse height of the Back scintillator alone was the appearance of distinct regions within the Forward distribution of neutron events. These bands appear at about channels 300 and 340 on the TOF axis as shown in Figure 2.44. This suggests that more than one mechanism contributes to the events in the Forward distribution. It is likely that the events in the band around channel 300 are caused by neutrons that interact first in the Front scintillator and then in the Back scintillator. The events near channel 340 may be caused by neutrons that interact in the Front scintillator and then reflect off the lead shield before interacting in the Back scintillator, thus resulting in a longer TOF.

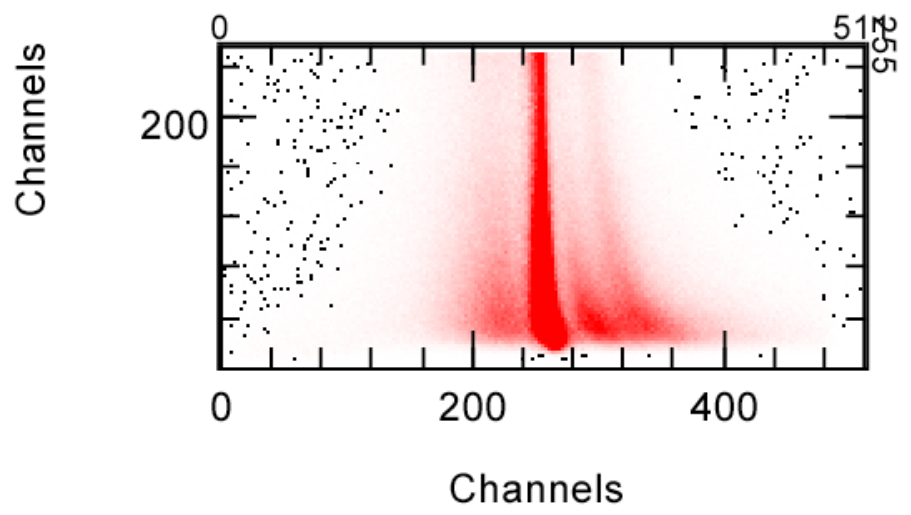
Figure 2.44. TOF (horizontal axis) Versus Pulse Height in the Back Scintillator
(vertical axis) for the ${ }^{252} \mathrm{Cf}$ Source with the TOF Discriminators at $100 \mathrm{mV}$

Another feature visible in Figure 2.44 is that the position of the TOF peaks in the Forward distribution changes as the energy of the event increases. This trend is expected because an event depositing more energy in the scintillator is likely to have been caused by a neutron with greater energy and thus a smaller TOF. A detailed look at the trend is available by plotting the TOF spectrum for a narrow band of channels on the energy axis. Figure 2.45 shows an example of one such slice. 


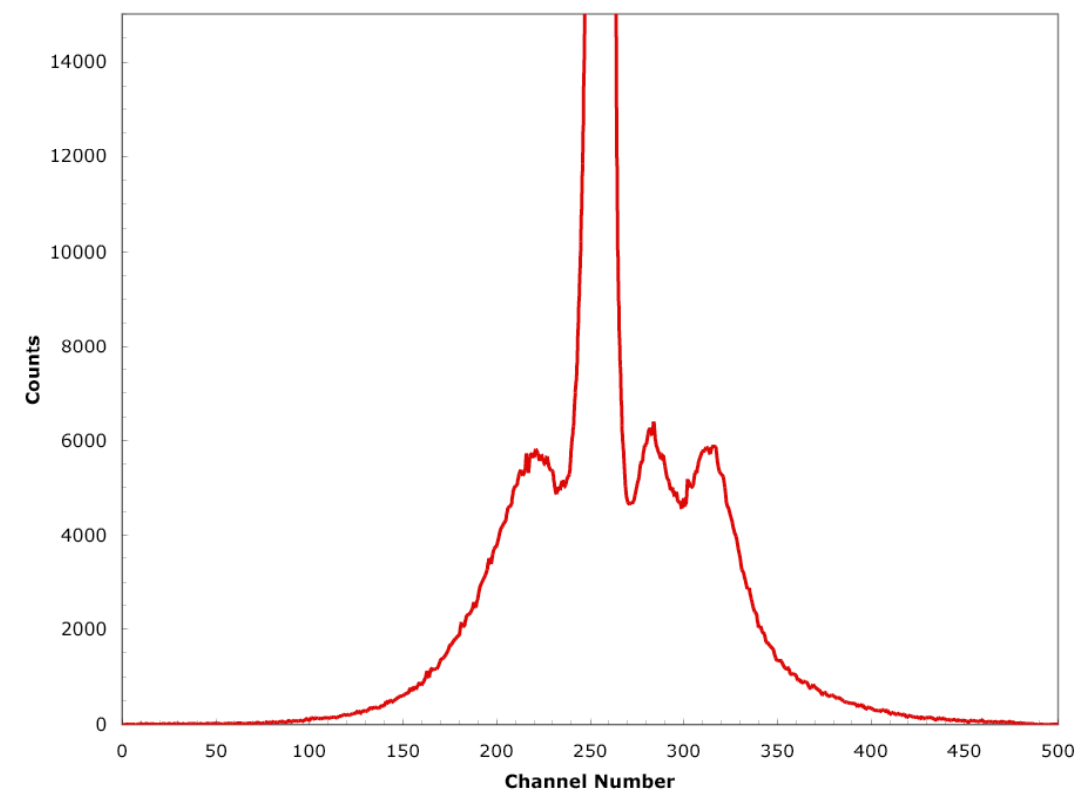

Figure 2.45. TOF Spectrum Gated by a Narrow Region (channels 70-89) on the Back Scintillator Pulse-Height Axis

Figure 2.46 shows the location of the neutron peaks on the TOF axis as a function of the energy bin for the Back scintillator. The peak location does not shift much for events associated with the Reverse mechanism whereas both peaks in the Forward mechanism decrease rapidly as the deposited energy increases and then fall off more slowly. For the Forward mechanism, a lower channel number on the TOF axis corresponds to a higher energy neutron.

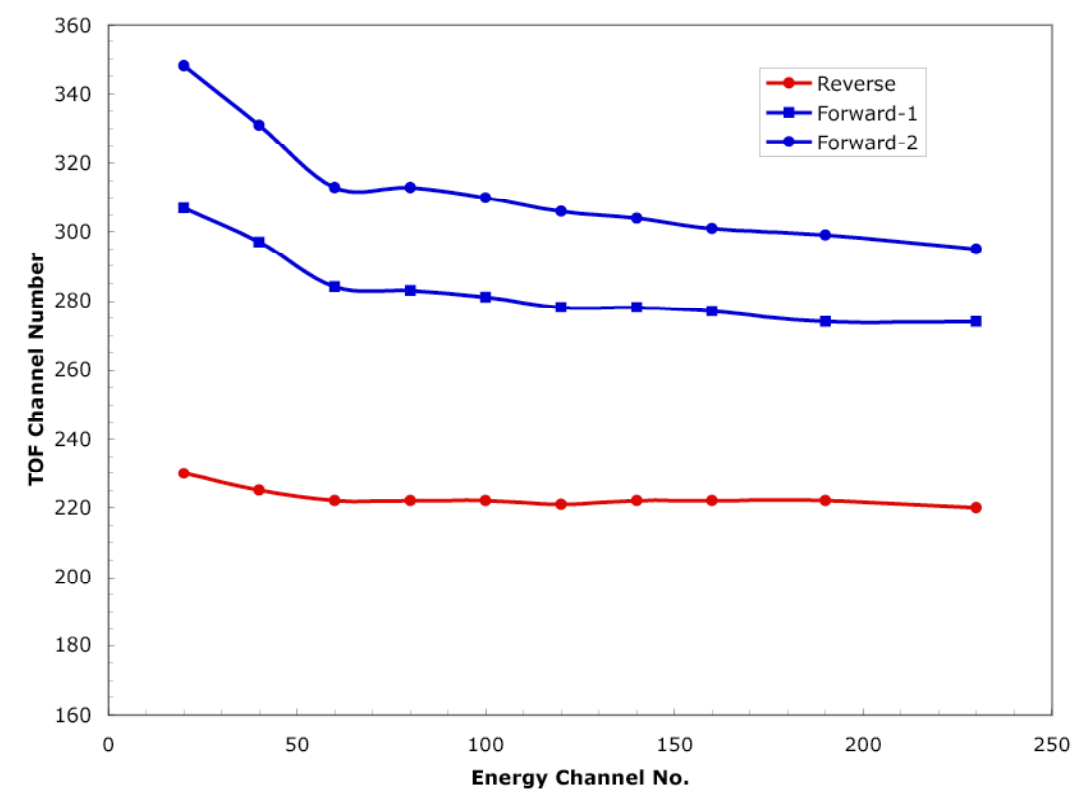

Figure 2.46. Location of Peaks on TOF Axis for TOF Spectra Gated by Narrow Bands on the Pulse-Height Axis for the Back Scintillator 
Quite different trends are observed for the location of the neutron peaks on the TOF axis when gated by increasing energy bins for pulse heights in the Front scintillator as shown in Figure 2.47. In this case, the location of the TOF peak does not change much for the Forward events whereas the location changes significantly for events in the Reverse mechanism. Note that when the peak location increases in the Reverse mechanism, it means that the neutrons are moving faster and correspond to higher energy neutrons. There is no evidence for two peaks on the TOF axis for the Forward mechanism when gating on pulse heights in the Front detector. This may be related to the fact that the Front scintillator is less well shielded by lead and sees fewer lead-scattered events.

The fact that the neutron TOF distributions evidently reveal several contributing mechanisms reinforces the conclusion that precise neutron spectroscopy on an event-by-event basis is not feasible with the current ALPS II sensor using the TOF information alone. Timing information alone does permit efficient fast neutron detection and effective discrimination of neutron from gamma events. Further development could lead to a simultaneous measure of neutron energy spectra based on summed pulse heights in the two detectors. Alternatively, a more precise method of measuring the interaction position within each scintillator (or a subdivision of each monolithic scintillator sheet into limited-width strips, each outfitted with a pair of PMTs) would be required to yield a timing-based measurement of particle velocity.

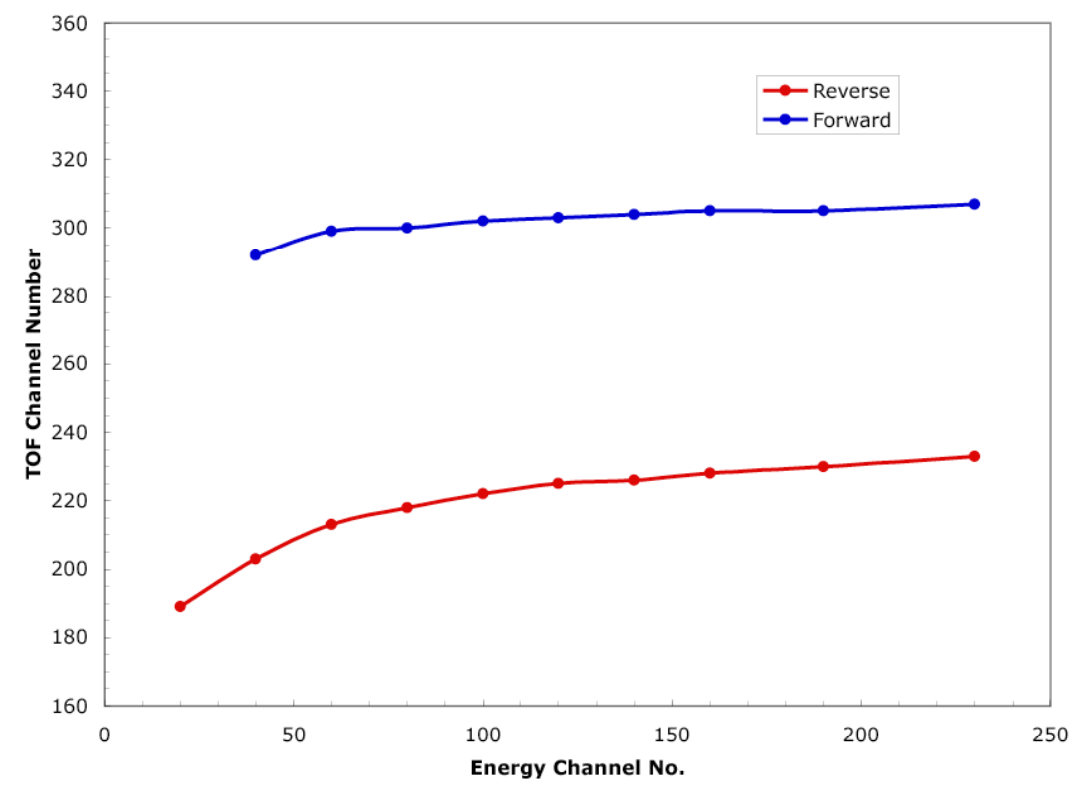

Figure 2.47. Location of Peaks on TOF Axis for TOF Spectra Gated by Narrow Bands on the Pulse-Height Axis for the Front Scintillator

\subsection{Experimental Campaign: Conclusions}

The data presented here are intended to benchmark computer models that might be used to design a fast neutron sensor optimized for efficient detection in a specific application. Referring back to the TOF spectrum for the $30 \times 30 \mathrm{~cm}^{2}$ sensor displayed in Figure 1.1, we anticipate that moving the two scintillator slabs farther apart (i.e., increasing the ratio of separation distance to a characteristic size of each scintillator) will increase the separation between neutron and gamma events in the TOF spectra and thus 
allow greater discrimination of gammas from fast neutrons. The consequence of moving the scintillators farther apart is a lower detection efficiency. Thus, the optimum conditions might best be determined by computer modeling.

In a field instrument without the lead shield, only positive time intervals will be available for detecting neutrons. A positive time threshold will be set below which no events are counted. The efficiency for detecting gammas should be based on the entire gamma distribution, including both positive and negative time intervals. However, only that portion of the gamma distribution above the positive time threshold will contribute a gamma contamination to the number of neutron counts.

The present system does not allow a reasonably precise measurement of the neutron energy spectrum after scattering. This is because the length of the flight path is not well determined by the interaction position information available from each scintillator, precluding an accurate correction to the nominal (normal-incidence) flight path and calculation of a realistic velocity. By supplementing the TOF measurement with PMT pulse height information, it should be possible not only to identify fast neutrons by their TOF, but also to simultaneously measure the energy of the initial neutron by summing the pulse outputs from both scintillator slabs. 


\subsection{Simulation}

Development of a Monte Carlo simulation code to model the neutron and gamma responses of the dualscintillator n-TOF sensor proceeded in parallel with the experimental campaign. Although the Monte Carlo N-Particle Transport Code (MCNP) represents the "gold standard" for neutron transport calculations in many detector modeling applications of relevance to the radiological and national security communities, the simulation code for this experiment was developed using the Geant4 radiation transport modeling suite. Geant4 (referred to colloquially hereafter as "G4") is a C++ based detector Monte Carlo package developed at CERN that supports a full set of standard electromagnetic physics processes (Compton scattering, photoelectric effect, and pair production) for electrons and photons in material media. Neutron interactions are modeled using G4's "high precision" neutron physics package, which includes a description of elastic scattering based on differential scattering cross sections drawn from the ENDSDF nuclear reactions database. The framework also supports optical photon transport for scintillator modeling, although this capability has not been exploited to date in the n-TOF sensor model. The two primary goals of the simulation work were as follows:

- Guide interpretation of the neutron and gamma TOF spectra measured in the experimental campaign

- Develop a modeling tool appropriate for optimizing application-specific n-TOF sensor design, given satisfactory benchmarking against the experimental data.

The primary driver for choosing G4 rather than MCNP in this instance is the ease with which individual interactions, and the timing information associated with these interactions, can be stored, retrieved, and manipulated by the user. In contrast to MCNP, a stochastic modeling tool tailored primarily for the calculation of (for example) particle current or flux integrals and various energy-weighted or crosssection weighted variations on these integrals, G4 is inherently a particle-tracking simulation code. The G4 framework provides the user with convenient access to all relevant kinematic and material-interaction data associated with the tracking of an individual particle and the secondary particles produced in reactions suffered by a particle as it traverses a medium. This type of step-by-step tracking information, while available from MCNP using the PTRAC output option, typically requires parsing of an ascii-format output file and post-processing of the extracted particle history information. A G4 application typically involves storage of the step-by-step tracking info in data structures that the user's application-specific software retrieves at the end of each event, and this tracking data can then be manipulated and histogrammed as desired on-the-fly as a simulation "run" proceeds. In addition to providing a convenient framework for processing track information, the G4 physics suite also supports optical photon transport, so that it is possible in principle to "fully" model a scintillation-based TOF sensor from energy deposition by elastic scattering to calculation of light collection at the sensor's PMTs. In practice, G4's optical photon capability has not been exploited in the results reported here, primarily in an effort to avoid the significant computational overhead required for transport of the many UV photons $(\sim 10,000$ per MeV of deposited energy in a typical plastic scintillator) generated in the interactions of a single fast neutron with a scintillator.

It must be noted that the primary disadvantage to the choice of G4 for the n-TOF sensor simulation is that the "track record" of G4's neutron physics is not nearly as well established as that of MCNP's neutron physics. For the purposes of a TOF sensor simulation, however, one expects elastic scattering of fast 
neutrons (in the scintillators themselves and in shielding materials constituting the sensor housing) to be the predominant interaction mechanism governing the sensor response. Thus, a radiation transport package that can handle fast neutron elastic scattering with reasonable accuracy should suffice for this modeling application, and so G4 (incorporating the so-called "high-precision," or "HP," neutron physics set of classes) was chosen as the application development framework.

Simulating a TOF variable that can be meaningfully compared to data requires some care. As noted above, a realistic model of the experimental apparatus should account not only for primary interactions (e.g., elastic scattering) of the incident neutron within each of the plastic scintillators, leading to spatially well-defined sites at which finite energy deposition in the detector medium occurs, but also for the propagation of scintillation photons from these energy-deposition sites to the PMTs and for the formation of the PMT output signal. In this way, a "full" simulation of the pulse set processed by the sensor's readout electronics can in principle be achieved, yielding a model that faithfully describes the time development of each PMT signal participating in the sensor's event-selection trigger. The pseudo-signal processing incorporated in a full-scale simulation can then be constructed to reproduce the processing of data registered in the apparatus. This approach permits calculation of a simulated "TOF" variable that matches as closely as possible, insofar as its definition in terms of calculations performed upon "raw" quantities (i.e., TDC values) is concerned, the TOF quantity measured in the experimental campaign.

In practice, a substantially simpler computational strategy was adopted for calculating the simulated TOF variable described here, primarily to avoid the prohibitive central processing unit (CPU) overhead required for individual scintillation photon tracking. For each simulated incident neutron (or gamma) history, an energy-weighted mean interaction time in each scintillator is computed from a set of $\mathbf{M}$ tracking "hits" (where a "hit" here refers to a discrete, non-zero energy deposition event in the detector medium at a specified spatial point and at a specified time in the laboratory system, as reported by the tracking framework) according to the following formula:

$$
\overline{\mathbf{T}}_{\mathbf{i}}=\frac{\sum_{\mathrm{j}=1}^{\mathrm{M}} \mathbf{T}_{\mathrm{ij}} \Delta \mathrm{E}_{\mathrm{ij}}}{\sum_{\mathrm{j}=1}^{\mathrm{M}} \Delta \mathrm{E}_{\mathrm{ij}}}, \quad \mathbf{i}=\mathbf{1 , 2}
$$

where $\overline{\mathbf{T}}_{\mathbf{i}}$ is the mean interaction time in scintillator $\mathrm{i}, \Delta \mathbf{E}_{\mathbf{i j}}$ is the energy deposition in hit $\mathrm{j}, \mathrm{j}=1, \ldots, \mathrm{M}$, in scintillator $\mathrm{i}$, and $\mathbf{T}_{\mathrm{ij}}$ is the time (in the laboratory system) at which energy deposition $\Delta \mathrm{E}_{\mathrm{ij}}$ occurs.

Note that the mean interaction time thus corresponds to the energy-weighted mean time of energy deposition in the scintillator. In the event that a neutron suffers only a single elastic collision within a given scintillator, leading to energy deposition by only a single recoiling ion, the mean interaction time will equal the time assigned by the tracking framework to this single energy deposition hit. If, on the other hand, an incident neutron undergoes multiple interactions in the scintillator, or (for a sufficiently energetic incident neutron) ejects secondary neutrons via one or more inelastic nuclear reactions, the mean interaction time calculated for this event will correspond to the mean time of the set of energy deposition hits from all neutron interactions, weighted by the energy deposition in each hit. The motivation for this definition of the mean interaction time is to construct a variable that suggests the time development of each scintillator's output pulse without actually performing the optical photon transport 
required to produce a "realistic" pulse model. The simulated TOF variable is then calculated as the difference of the scintillator 2 and scintillator 1 energy-weighted mean times.

The simulation geometry comprises the following elements:

- Two plastic scintillator slabs of dimensions and separation matching those of the apparatus (namely, $127 \mathrm{~cm} \times 57.2 \mathrm{~cm} \times 5.08 \mathrm{~cm}$, slabs at $17.8 \mathrm{~cm}$ center-to-center separation)

- $\mathrm{A} \mathrm{Pb}$ absorber sheet of thickness $0.32 \mathrm{~cm}$, sandwiched between two sheets of $0.635-\mathrm{cm}$ thickness polycarbonate and positioned mid-way between the two scintillators

- $\mathrm{Pb}$ shielding walls of thickness $5.08 \mathrm{~cm}$ enclosing the detector package on four sides (rear, bottom, and sides), dimensioned and positioned relative to the package as per CAD renderings of the experimental apparatus

- A steel plate of thickness $2.54 \mathrm{~cm}$ mounted $14 \mathrm{~cm}$ in front of the first scintillator.

The simulation's user input front-end supports specification of a variety of source terms, including gamma and neutron particle types, mono-energetic or lookup table-sampled energy distributions, point or extended source position distributions, and several angular distribution options, including isotropic emission and uni-directional (or "pencil beam") emission. Simulation output includes a set of standard histograms of the TOF variable, energy deposition and "signal" timing in each scintillator, and diagnostic spectra of, for example, the generated energy distribution. In addition, the code provides the option for generating list-mode output of energy deposition and timing variables for further manipulation in the event that analysis beyond the standard histogram set is desired. In particular, post-processing of the listmode output provides a convenient means of examining the influence of ad hoc smearing of the scintillator signal timing before calculating the TOF variable, and for defining TDC histograms gated on cuts placed on the energy deposition in each scintillator (to mimic the influence of the experimental PMT discriminator thresholds).

\subsection{Simulation Results: Mono-Energetic Gamma Incident}

Figure 3.1 displays the simulated TOF spectrum for a monoenergetic gamma source, ${ }^{137} \mathrm{Cs}(662 \mathrm{keV})$. The width of the corresponding measured spectrum (see Figure 2.8) is significantly underpredicted by the effective mean scintillator time prescription of Equation (3.1). The FWHM of the main peak of the simulated spectrum is approximately $0.4 \mathrm{~ns}$ in comparison to the measured value of $4.5 \pm 0.7 \mathrm{~ns}$. The sensor's gamma response should be dominated by "single-site" scattering events (e.g. Compton scattering in the first scintillator with a second scattering in the second scintillator), for which the prescription (Eq. 3.1) simply reproduces the timing of the single interaction. Thus, the difference between the simulated and observed TOF peak widths isolates the contributions of 1) scintillation light generation and propagation and 2) PMT pulse formation, to the measured timing dispersion. The small "satellite" peak visible in the TOF distribution at negative time evidently corresponds to Compton back-scattered events from either the $\mathrm{Pb}$ shielding or the second scintillator. For these events, the gamma traverses the front scintillator in the "front to back" direction without interacting, scatters from a structure at the rear of the sensor (either the $\mathrm{Pb}$ shielding wall or the back scintillator), and then deposits energy as it travels in the "back to front" direction to yield scintillator hits in the time sequence (back scintillator, front scintillator). 


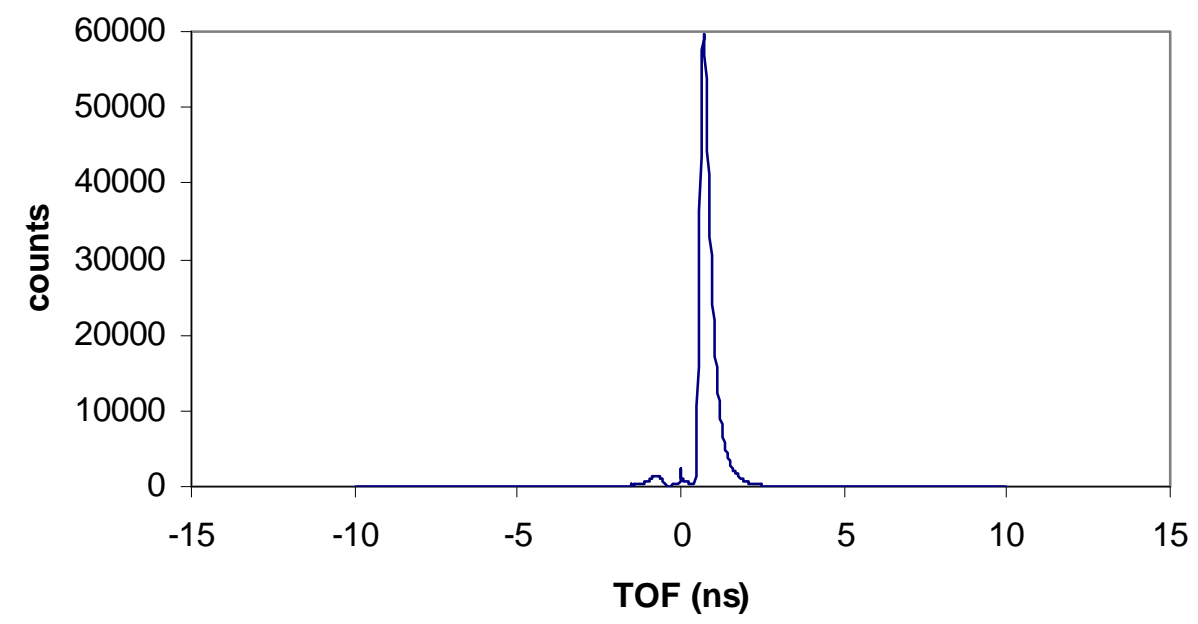

Figure 3.1. Simulated ${ }^{137}$ Cs Gamma TOF Spectrum for the Dual-Sheet Sensor

The dispersion in each scintillator's signal time required to account for the measured TOF width has been investigated in the simulation by applying Gaussian smearing to each of the scintillator times calculated via (Eq. 3.1) before taking the difference of these times to form the simulated TOF variable. The smeared TOF distribution thus obtained is compared to data in Figure 3.2. (The $100-\mathrm{mV}$ threshold data are shown in this figure, but the gamma peak width is essentially independent of the threshold for the 10-, 20-, 50-, and $100-\mathrm{mV}$ values measured.) The standard deviation of the Gaussian component that yields the best match to the width of the measured spectrum is $1.3 \mathrm{~ns}$. Because the neutron TOF spectrum has significant strength over an interval of at least $100 \mathrm{~ns}$, neglecting this contribution to the timing dispersion represents a tolerable approximation in modeling the sensor's neutron response. Clearly, the simulated TOF spectrum with this ad hoc smearing procedure applied does not reproduce the asymmetry of the measured TOF peak. Failure to reproduce the gamma TOF peak shape in detail will compromise the reliability of simulated gamma rejection fraction as a function of TOF threshold channel. Thus, for a design optimization study in which data would not in general be available to calibrate the time smearing (as has been done here), it would be advisable to apply a range of individual scintillator time signal dispersions, spanning values from 1 to $2 \mathrm{~ns}$, to bound the expected performance of the system.

Figure 3.3 compares the sensor's intrinsic gamma detection efficiency (summed over the entire ${ }^{137} \mathrm{Cs}$ TOF distribution) for the simulation and data. The "intrinsic" efficiency refers to the detection efficiency for particles incident on the front face of the sensor, i.e., a geometric factor corresponding to the ratio of the solid angle subtended by the sensor to $4 \pi$ ( 0.35 for the simulated source/sensor configuration) has been divided out of the simulation's absolute efficiency. The simulation's gamma detection efficiencies are plotted as a function of a threshold cut on the energy deposition in each scintillator; i.e., both scintillators must have an energy deposition of at least the threshold value to yield a valid sensor TOF event in the simulation. The corresponding intrinsic efficiencies in the data are plotted as a function of the electronic pulse-processing discriminator threshold for each contributing PMT in the apparatus. The figure shows that the intrinsic detection efficiency of the simulated sensor can be reconciled with that of the apparatus if the $10-\mathrm{mV}$ discriminator threshold (for example) yields an effective single-scintillator energy deposition threshold of approximately $70 \mathrm{keV}$. Note that this threshold correspondence must be interpreted within the context of the coincidence requirements imposed. In the data, four PMTs (two at each end of both scintillators) must generate a pulse exceeding the readout electronics threshold whereas in the simulation, both scintillators must register deposited energy exceeding the software threshold. 


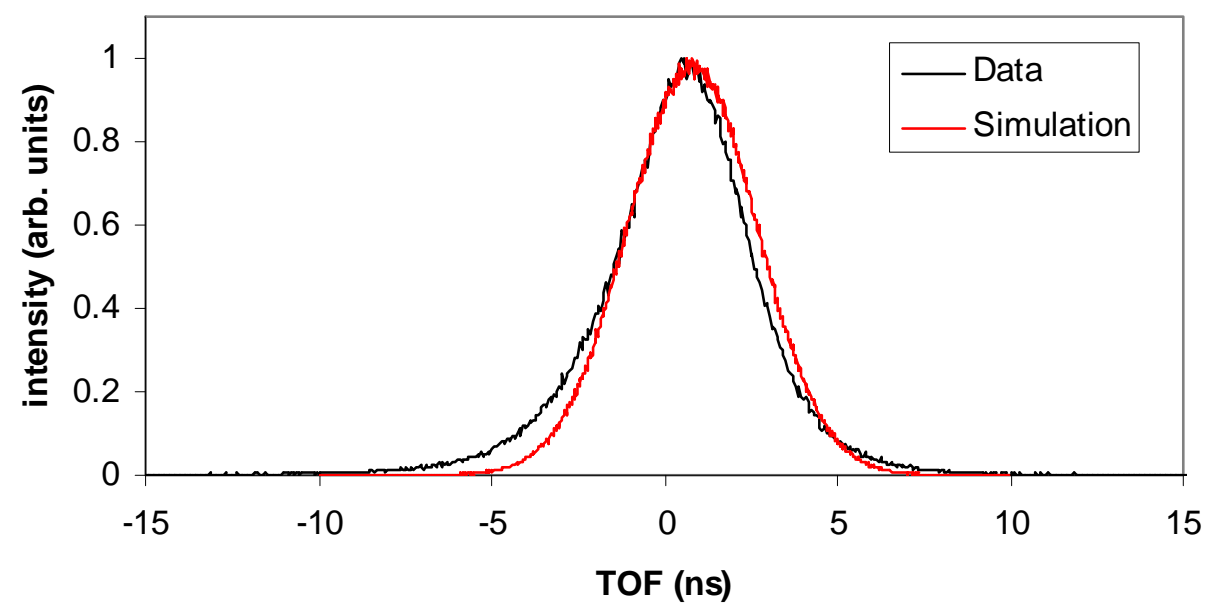

Figure 3.2. Comparison of Simulated ${ }^{137}$ Cs TOF Spectrum (with 1.3-ns Gaussian smearing applied to both scintillator times before calculating TOF) to Data. The "spike" at channel 0 of the data spectrum, visible in Figure 2.8, has been removed for this figure. Both spectra are normalized to unity at the maximum channel for the purposes of comparing the distribution shapes.

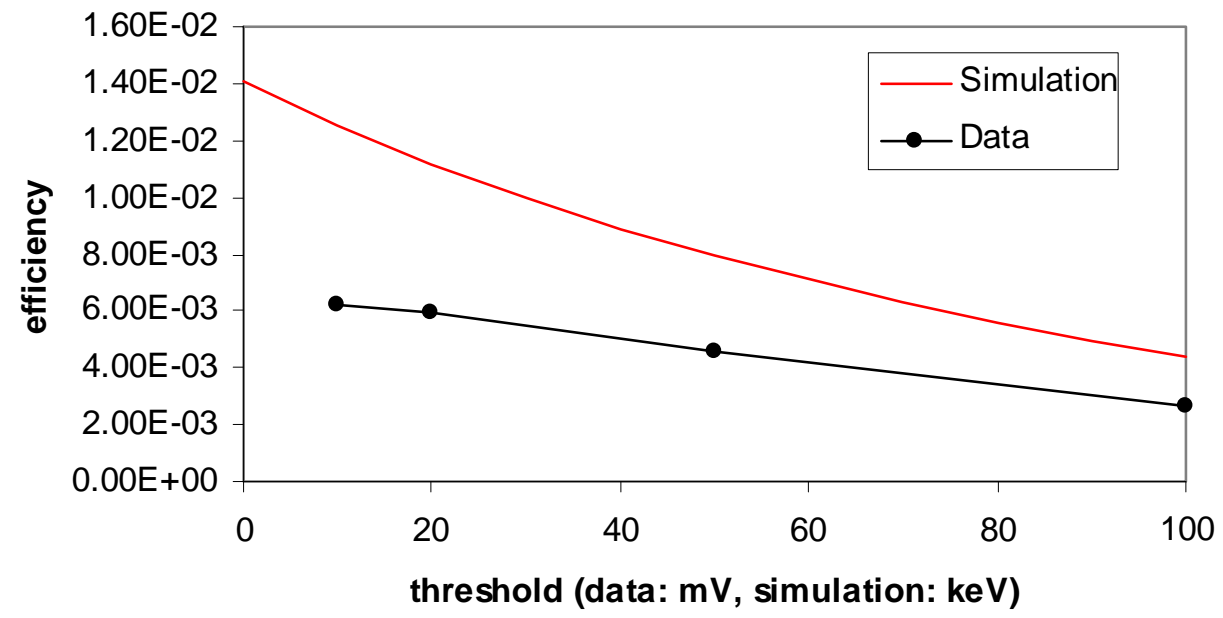

Figure 3.3. Intrinsic Sensor Gamma Detection Efficiency as a Function of Threshold for the Data and the Simulation

\subsection{Simulation Results: ${ }^{252} \mathrm{Cf}$ Neutron Source}

The simulated response of the TOF sensor to a ${ }^{252} \mathrm{Cf}$ spontaneous fission neutron spectrum source term (displayed in Figure 3.4) is depicted in Figure 3.5. The TOF spectra in this figure correspond to the simulation output with and without a 1.3-ns Gaussian smearing applied to the individual scintillator timing signals. Note that the source term does not include explicitly generated gammas, so that the gamma "flash" peak visible near zero TOF arises from secondary gammas produced by the G4 radiation transport framework (e.g., the $2.2-\mathrm{MeV}$ gamma produced by neutron capture on hydrogen). 


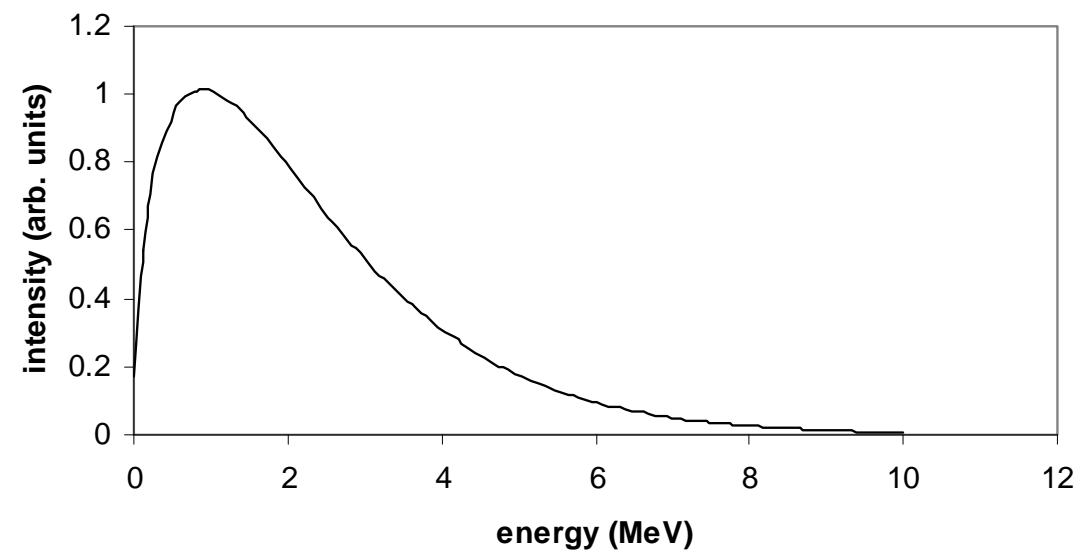

Figure 3.4. ${ }^{252}$ Cf Spontaneous Fission Neutron Spectrum Used as the Source Term for the n-TOF Sensor Response Simulation

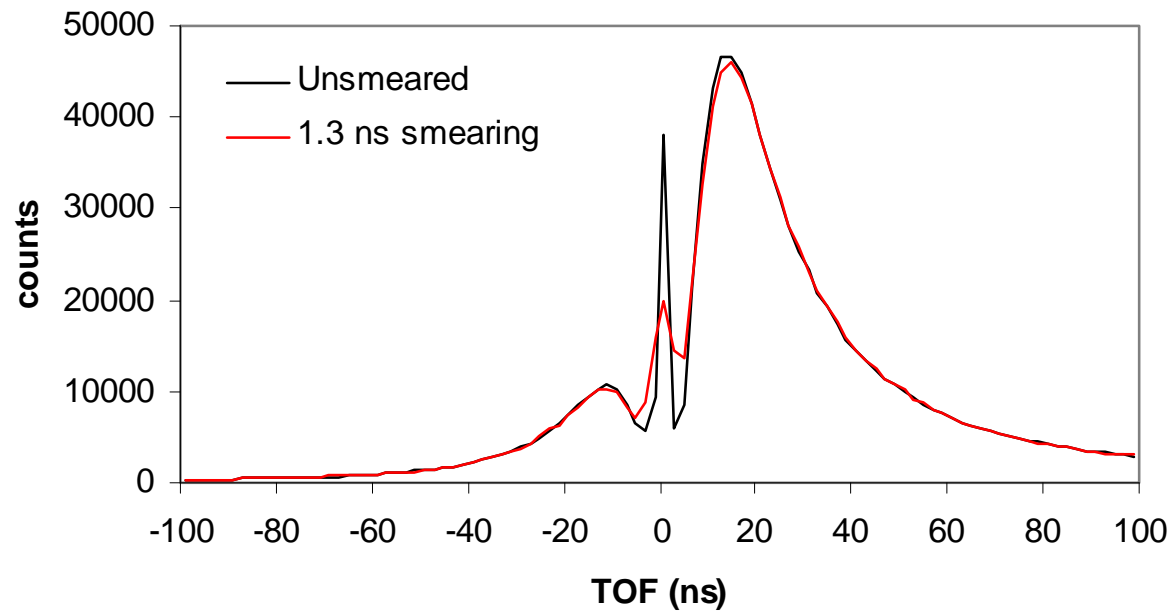

Figure 3.5. Simulated ${ }^{252} \mathrm{Cf}$ Neutron Spectrum Without (black curve) and with (red curve) Gaussian Smearing (1.3-ns standard deviation) Applied to the Time Signals from Both Scintillators

In Figure 3.6, the simulated ${ }^{252} \mathrm{Cf}$ TOF spectrum is overlaid on the measured spectrum at the $10-\mathrm{mV}$ discriminator threshold. Both spectra are normalized to unity at the channel, corresponding to the most probable TOF value. The following features are readily apparent:

- The model reproduces the qualitative nature of the "forward" or "front to back" (i.e., positive TOF) portion of the neutron distribution with reasonable fidelity, although it fails to reproduce the detailed shape of this distribution. The most probable TOF value in the "forward" portion of the simulated distribution is shifted to about $5 \mathrm{~ns}$ earlier time than observed in the data. A comparison of the data and simulation shapes for the "forward" distribution is displayed in an alternative format in Figure 3.7, which depicts the integrated intensity above the TOF threshold, as a function of the TOF threshold channel. Both of these curves are normalized to unity at the minimum TOF threshold, so that only the difference in the curve shapes is relevant. The simulated spectrum has significantly greater strength at large TOF (40 to $80 \mathrm{~ns}$ ) than is observed in the data. 
- The model grossly underpredicts the strength of the "reverse" or "back to front" portion of the TOF distribution relative to the "forward" portion. In the data spectrum for the $10-\mathrm{mV}$ discriminator threshold, the ratio of reverse ( $-90 \mathrm{~ns}$ to $-5 \mathrm{~ns})$ to forward ( $5 \mathrm{~ns}$ to $90 \mathrm{~ns}$ ) events is approximately 0.46 . This ratio is only 0.19 for the simulated spectrum, i.e., the simulation underpredicts the strength of the reverse distribution relative to the forward distribution by a factor of 2.5 .

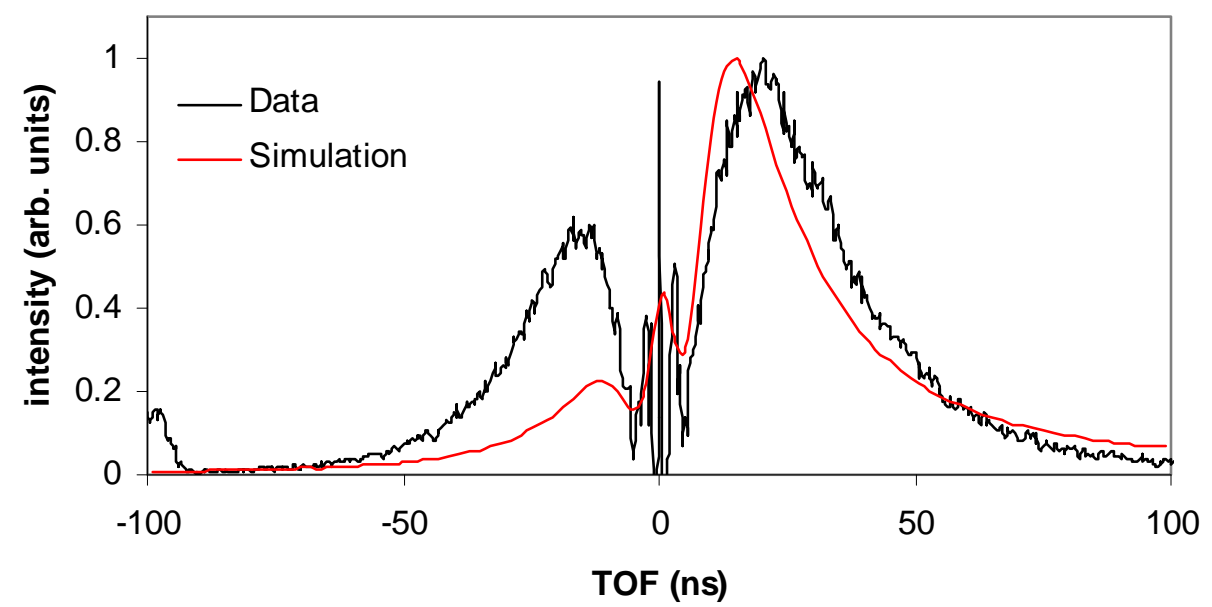

Figure 3.6. Comparison of Simulated ${ }^{252}$ Cf Neutron TOF Spectrum to Measured Spectrum. Both spectra are normalized to unity at the channel corresponding to the most probable TOF value. The data were recorded with 10-mV discriminator thresholds.

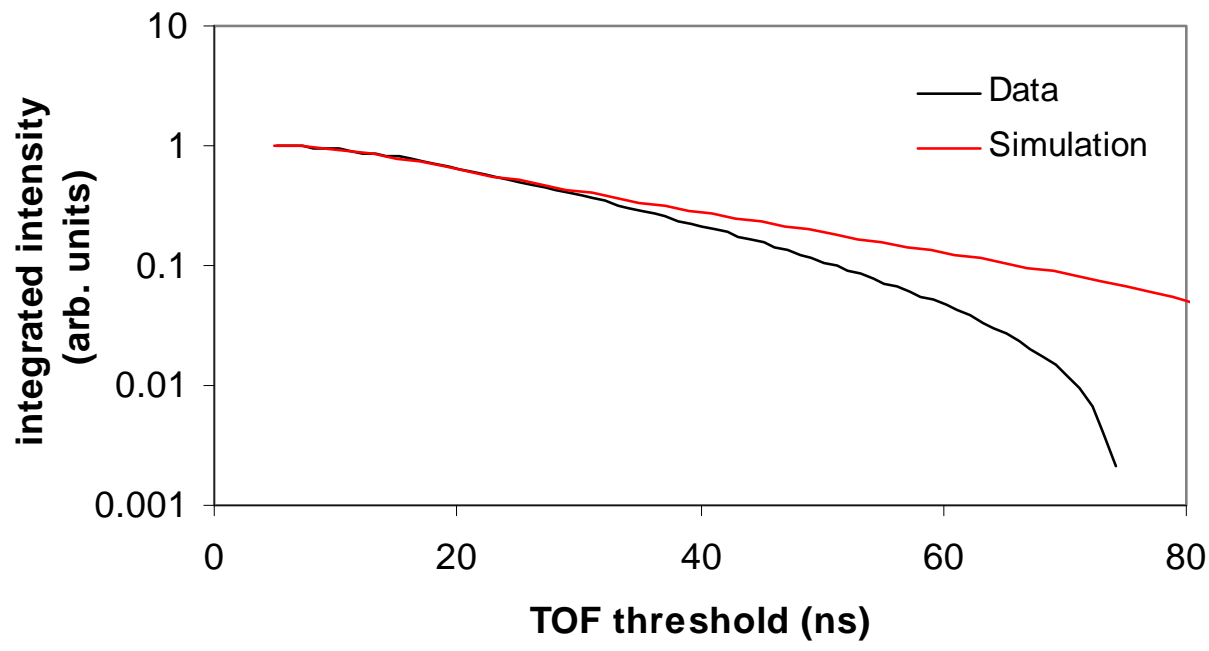

Figure 3.7. Integrated Intensity Above TOF Threshold for the "Forward" Portion of the Sensor's ${ }^{252} \mathrm{Cf}$ Neutron TOF Distribution in Data (10-mV threshold) and Simulation. Both curves have been normalized to unity at a TOF threshold of 5 ns.

The failure of the model to reproduce the intensity of the "reverse" TOF distribution has not yet been satisfactorily explained. One possibility that suggested itself immediately is that the G4 neutron physics framework underpredicts the back-scattering of neutrons from the rear $\mathrm{Pb}$ shielding wall of the detector hut. To address this issue, a comparison modeling study of fast neutron reflection from a spatially 
isolated, 5.08-cm thick, $1-\mathrm{m}^{2}$ slab of $\mathrm{Pb}$ was conducted using identical geometries in two radiation transport codes, MCNP4c and an appropriately adapted version of the n-TOF sensor G4 code. In both cases, the reflected neutron flux passing through a "detector" volume $\left(1 \mathrm{~m}^{2}\right.$ in area $\times 1 \mathrm{~mm}$ thickness $)$ at $1-\mathrm{m}$ standoff distance from the $\mathrm{Pb}$ slab was calculated. The source terms for the simulations were monoenergetic, uni-directional "pencil beams" originating at a point between the slab and the detector volume and pointed toward the slab, so that any flux recorded in the detector volume must arise from a scattering event in the $\mathrm{Pb}$ slab. The two codes agreed in their prediction of the reflected flux (as integrated over the surface of the $1-\mathrm{m}^{2}$ detector volume) to within $10 \%$ over an energy range of $0.1 \mathrm{MeV}$ to $5 \mathrm{MeV}$, an interval that encompasses about $90 \%$ of the ${ }^{252} \mathrm{Cf}$ spontaneous fission spectrum. Thus, to the extent that MCNP4c serves as a reliable benchmark for neutron scattering calculations, the G4 framework appears to treat neutron reflections from a single $\mathrm{Pb}$ shielding slab with adequate precision for the purposes of the $\mathrm{n}$ TOF sensor simulation. The possibility remains that the angular dependence of elastic scattering in $\mathrm{Pb}$ is not reproduced accurately enough in the simulation to correctly model the return flux from multiple-wall interactions; no attempt has been made to investigate this possibility further.

A second possible explanation for the underprediction of the "reverse" neutron response observed in the data is that the energy-weighted mean time prescription adopted for the simulated TOF variable definition cannot adequately reproduce the coincidence timing requirement imposed by the pulse-processing electronics in the experimental apparatus. The coincidence requirement in the apparatus will tend to select correlated pairs of neutron interactions in the two scintillators because only PMT pulses adjacent in time (i.e., falling within a coincident time window) can satisfy the experimental data-acquisition trigger. This correlated-pair enforcement is missing in the prescription (Eq. 3.1), which simply sums over all hits depositing energy in a given scintillator, regardless of when they occur in time. To test whether the TOF variable prescription could be artificially distorting or suppressing the calculated reverse neutron response, the simulated neutron-induced singles rates in each scintillator were compared with and without the rear $\mathrm{Pb}$ shielding wall in place. The presence of the rear $\mathrm{Pb}$ wall enhanced the simulated neutron count rate in the rear scintillator by approximately $50 \%$, but only enhanced the forward scintillator singles rate by about $18 \%$. Because hits in both scintillators are required to define the TOF variable, gross accounting would suggest that the model would never predict more than an $18 \%$ enhancement in the "reverse" neutron response caused by the presence of the $\mathrm{Pb}$ wall, regardless of the approximations inherent in prescription (Eq. 3.1).

Given the inability of the simulation to reproduce the "reverse" neutron response, investigations of the simulation's neutron detection efficiency focused on the "forward" response, for which the simulation provides a qualitatively reasonable, if not perfect, account of the data. The intrinsic neutron detection efficiency for the "forward" distribution (integrated above a TOF threshold of $5 \mathrm{~ns}$ ) is displayed in Figure 3.8 as a function of either PMT discriminator threshold (data) or energy deposition threshold (simulation). By drawing a horizontal line through the two curves, one finds that the efficiency of approximately $4 \%$ observed in the data at $10-\mathrm{mV}$ discriminator threshold corresponds to an energy deposition threshold in the simulation of about $350 \mathrm{keV}$. Note that this threshold is a factor of 5 higher than the ${ }^{137} \mathrm{Cs}$ gamma-response threshold of $70 \mathrm{keV}$ derived from a similar comparison of the graphs plotted in Figure 3.3. 


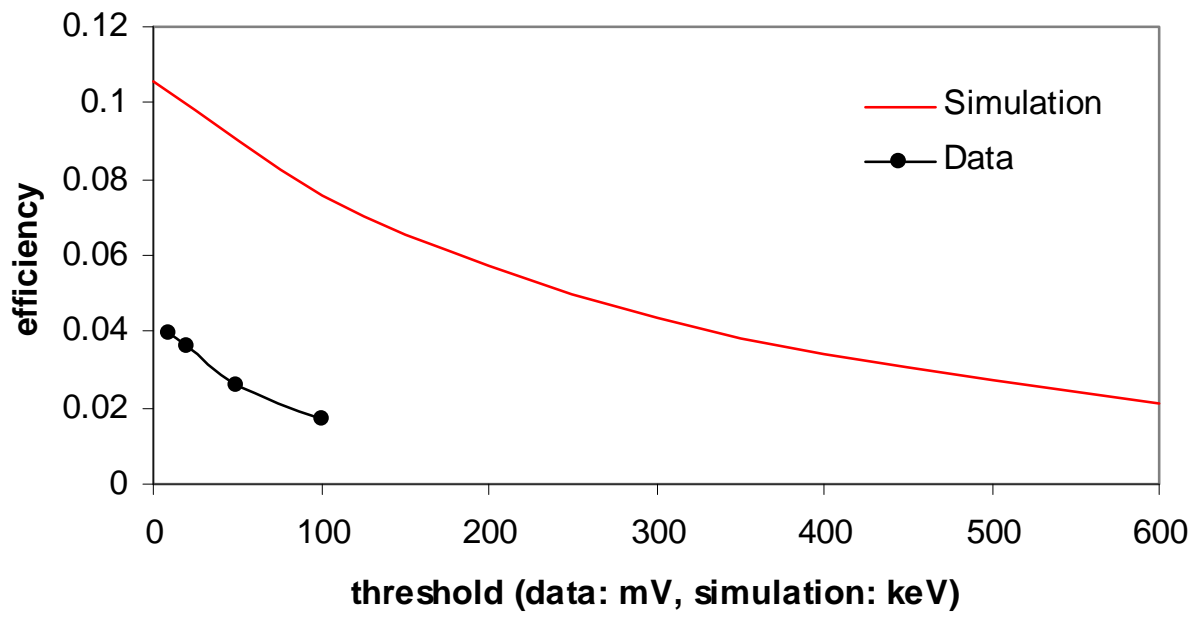

Figure 3.8. Intrinsic Sensor Neutron Detection Efficiency as a Function of Threshold for the Data and the Simulation. In both cases, the efficiency refers to the "forward" portion of the neutron TOF distribution, integrated above a TOF value of 5 ns.

This energy deposition threshold difference is not surprising because the light output of plastic scintillator when subjected to energy deposition by heavy ionizing particles (e.g., protons and ${ }^{12} \mathrm{C}$ ions, the main recoil products of elastic neutron scattering in plastic scintillator) is markedly smaller than the light response produced by fast electrons (i.e., the secondary products of gamma interactions) depositing the same amount of energy. The relative (electron, proton) light output responses of NE102 plastic scintillator (essentially identical to the BC-408 scintillator used in the n-TOF sensor) can be derived from measurements reported in Craun and Smith (1970) and expressed as power laws in the deposited energy as follows:

$$
\begin{array}{ll}
R_{e}(E)=2000 E^{1.4} & \text {, electrons } \\
R_{p}(E)=240 E^{1.7} & \text {, protons }
\end{array}
$$

In these formulas, the energy $\mathrm{E}$ is expressed in $\mathrm{MeV}$, and the scintillation light response $\mathrm{R}$ is expressed in units that are arbitrary but consistent between the two particle types. The formulas provide reasonable representations of the data sets in Craun and Smith (1970), which span the energy range from about 400 $\mathrm{keV}$ to $1 \mathrm{MeV}$ (gamma) and $300 \mathrm{keV}$ to $10 \mathrm{MeV}$ (proton). According to the electron response formula, a threshold of $70 \mathrm{keV}$ in the ${ }^{137} \mathrm{Cs}$ source case corresponds to a light output response $\mathrm{R}_{\mathrm{e}}$ of about 48 units. To obtain the same (recoil) proton-induced light output, $R_{p}$, a proton energy of $390 \mathrm{keV}$ is required according to the second formula. This value is in reasonable agreement with the simulated energy deposition threshold of about $350 \mathrm{keV}$ that reproduces the measured ${ }^{252} \mathrm{Cf}$ neutron efficiency. Of course, this simple analysis neglects the contributions of higher-mass recoil ions to the scintillator light output under exposure to fast neutrons (and these heavier species will tend to generate even less light per unit of energy deposited than protons). But it should be clear that this type of light-output argument provides a plausible explanation to reconcile the simulated energy deposition thresholds required to reproduce the measured gamma and neutron intrinsic detection efficiencies.

Figure 3.9 compares the intrinsic detection efficiencies for data and simulation as a function of TOF threshold over which the sensor response is integrated. The data correspond to the $10-\mathrm{mV}$ "forward" 
distributions previously presented in Figure 2.38. Note that the non-gaussian tail visible in the measured

${ }^{137}$ Cs gamma TOF distribution (see Figure 3.2) yields gamma "contamination" of the neutron distribution at values of the TOF threshold approximately 10 to $15 \mathrm{~ns}$ larger than predicted by the simulation.

Evidently, the time-smearing prescription applied to the simulated scintillator timing signals does not reproduce the mono-energetic gamma response shape accurately enough to permit a reliable prediction of the sensor's gamma rejection in this tail region. At a TOF threshold of $5 \mathrm{~ns}$, the simulation agrees with the data that the ratio of neutron to gamma efficiencies is approximately 100:1, and that as this threshold approaches $20 \mathrm{~ns}$, the rejection improves to better than $10^{4}: 1$. The rate at which the rejection ratio improves with increasing TOF threshold over the range 5 to $20 \mathrm{~ns}$, however, is significantly overestimated by the simulation.

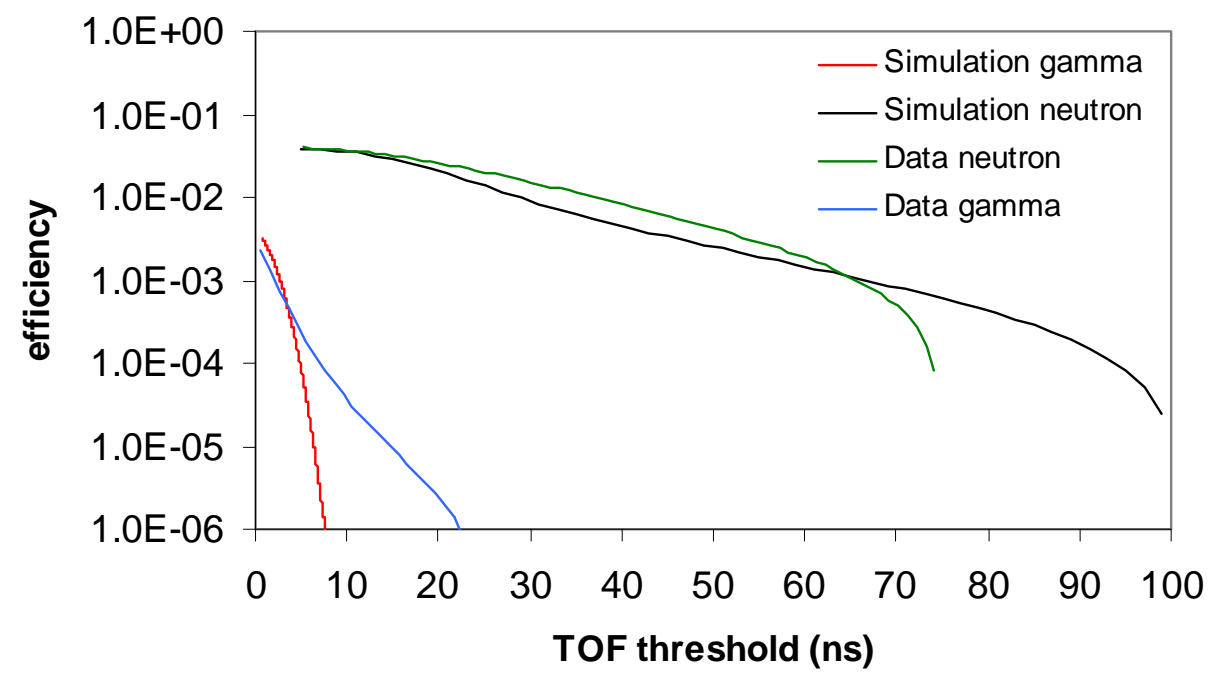

Figure 3.9. Intrinsic Detection Efficiency (detector response integrated above TOF threshold) as a Function of TOF Threshold for Gammas $\left({ }^{137} \mathrm{Cs} 662 \mathrm{keV}\right)$ and Neutrons $\left({ }^{252} \mathrm{Cf}\right)$. The data sets were recorded at 10-mV discriminator threshold and correspond to the "forward" sets in the 10-mV panel of Figure 2.38. The simulation results correspond to energy deposition thresholds of $70 \mathrm{keV}$ (gamma) and $350 \mathrm{keV}$ (neutron).

\subsection{Simulation: Conclusions}

The main conclusions of the n-TOF sensor simulation study can be briefly summarized as follows:

- The Geant4 particle-tracking code framework, which allows the user access to the time associated with energy deposition events (or "hits") in the detector medium, permits calculation of an effective, energy-deposition weighted TOF variable that can be meaningfully compared to data. The signal timing simulation in this approach neglects the propagation of scintillation light through the detector and the formation of a "realistic" PMT output pulse. The primary advantage of this approach is the savings in computational overhead required for individual scintillation photon tracking (a capability that is also available in G4, but was not exploited for this application).

- The simulation underpredicts the width of the sensor's mono-energetic gamma TOF distribution (662 keV incident energy) unless an ad hoc gaussian smearing is applied to the energy-weighted 
mean times of both scintillators. The standard deviation of this gaussian component is approximately $1.3 \mathrm{~ns}$. In contrast to the gamma case, this smearing has a negligible impact on the shape of the neutron TOF distribution, which spans a time range two orders of magnitude larger than the gaussian component's width. Non-gaussian tails in the measured gamma TOF response are not reproduced in this gaussian smearing approach, and thus the rate of change of the sensor's gamma rejection (i.e., the ratio of gamma to neutron efficiencies) as a function of TOF threshold cannot be reliably estimated over the TOF range 5 to $20 \mathrm{~ns}$.

- The simulation reproduces the measured intrinsic gamma detection efficiency at $662 \mathrm{keV}$ upon application of an energy-deposition threshold of $70 \mathrm{keV}$ to each scintillator in the simulation. This threshold is consistent with that required to reproduce the sensor's measured ${ }^{252} \mathrm{Cf}$ neutron efficiency when the difference in scintillator light response between secondary electrons and recoil protons is taken into account.

- The energy-weighted timing prescription cannot reproduce the sensor's measured neutron TOF distribution in detail, but provides a reasonable qualitative picture of the "forward" (i.e., front-toback) portion of the neutron distribution. The model substantially underpredicts the strength of the "reverse" (i.e., back-to-front) distribution observed in the data, a component that presumably arises from one or more elastic scattering "reflections" of the incident neutron flux from the $\mathrm{Pb}$ shielding walls lining the neutron sensor housing.

The simulation code as it stands can be used as a rapid design optimization tool for a large-area, neutron TOF sensor. The primary value of this modeling tool is to predict gamma and neutron detection efficiencies as a function of parameters such as scintillator size and separation, external and internal shielding components, and electronic pulse-processing threshold. The reduction in computational overhead introduced by neglecting the scintillation photon tracking is bought at a price: even with ad hoc gaussian smearing of the simulated scintillator signals, the transition region in the TOF spectrum between 100:1 and 10,000:1 gamma rejection factors cannot be reliably mapped as a function of TOF threshold. However, the detection efficiency of the sensor outside of the non-gaussian tail region of mono-energetic gamma response is reasonably well modeled for both gamma and fast neutron particle types incident, with the caveat that the difference in scintillation light output for secondary electrons and recoil protons must be taken into account when correlating effective energy-deposition thresholds corresponding to a single discriminator setting in the pulse-processing electronics. 


\subsection{Conclusions and Outlook}

The primary goal of the "Methods and Instruments for Fast Neutron Detection" project was to investigate possible alternatives to traditional (i.e., moderator-based) fast neutron detection as an enabling technology in an active, or "near-passive," neutron interrogation system. Project activity focused on quantifying the capabilities and performance of a large-area $\left(\sim 0.7 \mathrm{~m}^{2}\right)$, dual-sheet, plastic scintillator-based neutron TOF sensor. The most important conclusions of the experimental campaign, which exploited existing apparatus at PNNL, can be summarized briefly as follows:

- An intrinsic fast neutron detection efficiency of $4 \%$ to $6 \%$, as averaged over the ${ }^{252} \mathrm{Cf}$ neutron energy distribution (and integrated over the entire neutron TOF distribution from about 5 ns to $80 \mathrm{~ns}$ ), is readily achievable with a sensor of this size. The range in efficiency quoted here represents the contribution of the "reverse," or Pb-wall reflected, component of the neutron TOF distribution, which can yield an increase in the sensor's total fast neutron response of roughly $50 \%$. The corresponding mono-energetic gamma efficiency $(662 \mathrm{keV})$ is approximately $0.5 \%$, integrated over the entire gamma TOF distribution. The gamma "flash" peak is approximately $5 \mathrm{~ns}$ in width (FWHM) and is located within a few ns of zero in the TOF distribution.

- The sensor's gamma-rejection ratio (i.e., ratio of the intrinsic neutron detection efficiency to the intrinsic gamma detection efficiency) is of the order of 10,000:1 when the sensor's response is integrated above a TOF threshold of $20 \mathrm{~ns}$. The sensor's intrinsic neutron detection efficiency (for the "forward" portion of the TOF distribution) drops from about $4 \%$ to $2.5 \%$ when this TOF threshold is imposed as a "neutron" particle identification criterion.

- The TOF variable serves as the most useful particle identification variable in a monolithic sensor of this size. Attempts to reconstruct the particle velocity from the TOF and a crude measurement of the (long-axis) interaction position within each scintillator, as derived from the time differences in the double-ended PMT readout for a given scintillator, failed to yield cleaner gamma discrimination than could be achieved on the basis of the TOF distribution alone. The reasons for this failure are twofold: 1) the long-axis position resolution based on the TCD time difference is only 5 to $10 \mathrm{~cm}$, and 2) the sensor's PMT configuration does not yield a reliable measure of the interaction position along the transverse (i.e., short-axis) dimension in the plane of the scintillator. Because the "level arm" for the TOF measurement is relatively short compared to the scintillator size, these limitations on the interaction position measurement precision can contribute substantially to the uncertainty in the particle flight path between the scintillator slabs.

Note that the trigger conditions employed throughout the experimental campaign required coincident firing of four PMTs, one at each end of both scintillators. This "double-ended" PMT readout method, coupled with mean-timing of the two PMT signals from a given scintillator's two ends, was implemented to minimize dispersion in the scintillator signal timing arising from the transit time of scintillation light across the scintillator. It is likely that the intrinsic detection efficiency could be improved significantly by either 1) relaxing the trigger definition to require that only a single PMT from each scintillator fire, or 2) increasing the number of PMTs at each scintillator end and requiring that only one of each end's PMT set presents a valid pulse to the trigger electronics. The price of scheme 1) is the potential for significant degradation of the scintillator signal timing dispersion, resulting in an increased width of the gamma TOF distribution and greater gamma contamination in the sensor's "neutron" channel. Similarly, the price of implementing scheme 2) is the potential for increased signal timing dispersion because a single PMT can 
no longer be counted on to provide the sensor timing fiducial (i.e., the start signal to the TDC). The impact of scheme 2) on the detection efficiency and gamma/neutron discrimination could be evaluated readily with the existing apparatus because each scintillator end is outfitted with three PMTs. However, in the interests of defining the cleanest (and most easily interpreted) experiment trigger to serve as a baseline in characterizing the sensor performance, only a 4-PMT trigger was investigated in this project.

An ideal approach to quantifying the potential utility of a TOF sensor in an active or "near-passive" interrogation system would involve experimental measurements (or at least a computer simulation) of the sensor response in the beam environment produced by a neutron generator, as configured in a realistic sample-interrogation geometry. An important goal of this type of evaluation, which has not been performed in the present project, would be to evaluate 1) gamma background rates and 2) accidental (i.e., uncorrelated) coincidence rates between the two scintillators. Note that because a TOF sensor exploits scintillator signal timing, rather than pulse-height information, the problem of pulse pileup (i.e., the superposition of sensor responses to two or more gammas in accidentally coincidence, mimicking the response to a single neutron) per se does not limit the total particle flux viewable by the sensor without substantially compromised performance. However, two types of rate-limiting effects are inherent to a TOF system: 1) accidental coincidences between scintillators that generate an event trigger and 2) "pre-emptive" TDC hits for one or more PMTs, leading to corrupted timing information for these PMTs for true coincidences.

In the first rate-limiting event category, two separate and physically uncorrelated particles traverse the two scintillators within the coincidence resolving time of the system and produce PMT pulses of sufficient amplitude to satisfy the event trigger. The accidental trigger rate for these events, $\mathrm{R}_{\mathrm{acc}}$, can be estimated using the formula

$$
R_{\text {acc }}=2 R_{1} R_{2} \Delta t_{\text {coin }}
$$

where $R_{1}$ and $R_{2}$ are the singles rates in scintillators 1 and 2, respectively, and $\Delta t_{\text {coin }}$ is the coincidence resolving time. The singles rates in turn can be calculated by the usual product of the source emission rate, geometrical acceptance factor, and intrinsic detection efficiency for a single scintillator. Figure 4.1 plots the accidental (gamma-induced) trigger rate as a function of point-source emission rate for the sensor dimensions used in this project, assuming 1-m standoff distance, 30\% single-scintillator gamma detection efficiency for each scintillator, and 100-ns coincidence resolving time. Note that the accidental trigger rate is manageable (about $40 \mathrm{~Hz}$ ) at $1 \mathrm{MHz}$ source emission rate. 


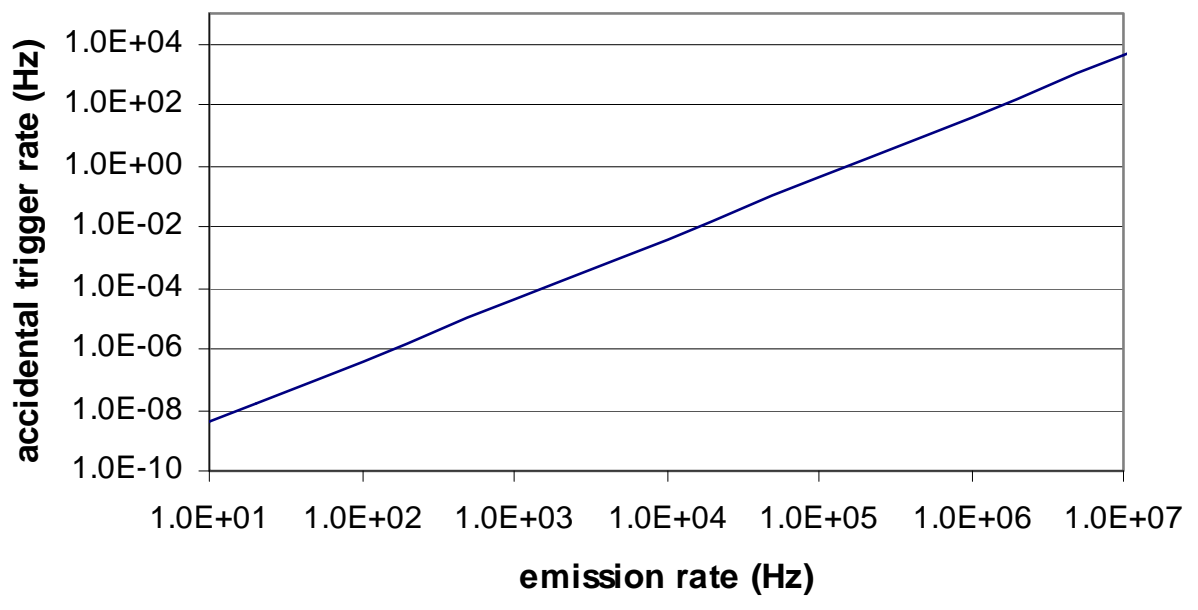

Figure 4.1. Calculated Gamma-Induced Accidental Trigger Rate as a Function of Point-Source Emission Rate for a TOF Sensor of the Same Dimensions as that Used in this Project. The front face of the sensor is at 1-m standoff from the point source.

In the second rate-limiting event category, if the singles rate from each PMT is too large, the measurement of times relative to the TDC timing fiducial, or "start signal," can be distorted by uncorrelated signals arriving at the TDC stop ahead of the set of signals that are actually associated with the particle generating the TDC start. The apparent time intervals registered in the TDC with respect to the fiducial will thus be too small, and the event is "stolen" from its true position in the time distribution for the given PMT and displaced to smaller times. The probability, $\mathrm{P}_{\text {stolen, }}$, that the TDC value for at least one of a set of N PMTs ( $=4$ in the case of the sensor used in this project) will be corrupted, or "stolen," from its correct position in an otherwise uncorrupted time distribution, in the course of readout for a truecoincidence event can be estimated via

$$
\mathbf{P}_{\text {stolen }}=\mathrm{NR}_{\mathrm{PMT}} \Delta \mathrm{t}_{\text {single }}
$$

where $\mathrm{R}_{\mathrm{PMT}}$ is the single-PMT rate, and $\Delta \mathbf{t}_{\text {single }}$ is an effective single-PMT pulse-resolving time determined by a combination of factors, including 1) the input pulse resolving time of the discriminator processing the PMT analog pulses, 2) the output pulse width of the discriminator, and 3) the maximum time interval (e.g., the maximum TOF) characterizing the upper limit on relevant time intervals registered in the TDC. For the purposes of a rough estimate, one can again assume that 50 to $100 \mathrm{~ns}$ is an appropriate range of values for this parameter, in which case the formula yields an event corruption probability of $2 \%$ to $4 \%$ at $100 \mathrm{kHz}$ single-PMT rate for a 4 PMT sensor. At $1 \mathrm{MHz}$ single-PMT rate, the corruption probability becomes unmanageably large at $20 \%$ to $40 \%$. It should be clear from these considerations that background rates are an important consideration in the application of a TOF sensor to an active interrogation system.

Although the neutron and gamma emissions in an active neutron interrogation system have not been simulated, the fast neutron detection efficiency of a TOF sensor comparable to that investigated in this project can at least be compared to the efficiency of a "baseline" ${ }^{3} \mathrm{He}$ system to establish the potential benefits realizable from DFND. With this goal in mind, the ${ }^{252} \mathrm{Cf}$ neutron detection efficiency was calculated using an MCNP model of a simple, but not completely unrealistic, moderated ${ }^{3} \mathrm{He}$ detector. A 
cross-sectional view of the detector model is displayed in Figure 4.2. The moderated detector consists of a row of five 10 -atmosphere ${ }^{3} \mathrm{He}$ tubes, each $5.08 \mathrm{~cm}(2 \mathrm{in}$.) in diameter by $1 \mathrm{~m}(3.3 \mathrm{ft})$ in length, sandwiched between two $1-\mathrm{m} \times 1-\mathrm{m} \times 1-\mathrm{cm}$ layers of polyethylene. The ${ }^{3} \mathrm{He}$ tube row is positioned midway between the two moderator layers, with the long axes of neighboring tubes separated by $20 \mathrm{~cm}$ (7.9 in.). The moderator slabs are separated by $5 \mathrm{~cm}$ ( 2 in.) (center-to-center). The intrinsic detection efficiency of this system was computed using a ${ }^{252} \mathrm{Cf}$ point source positioned $25 \mathrm{~cm}$ (10 in.) away from the front face of the first moderator slab, and a geometrical factor of approximately 0.3 (representing the "effective sensor area" presented by the front moderator slab) divided out of the absolute efficiency to compute the intrinsic efficiency. The MCNP calculation yields an intrinsic fast neutron efficiency of about $4.4 \times 10^{-3}$ for this sensor, or roughly a factor of 5 poorer than the n-TOF sensor's "forward" neutron response at 20 -ns TOF gamma-rejection threshold $(2.5 \%)$.

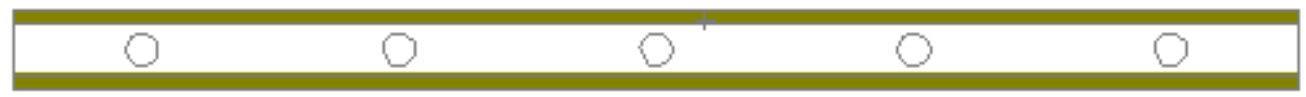

Figure 4.2. Cross-Sectional Schematic of Moderated ${ }^{3} \mathrm{He}$ Neutron Detector Modeled in MCNP. The ${ }^{3} \mathrm{He}$ tubes are $2.54 \mathrm{~cm}(1 \mathrm{in}$.) in diameter and positioned at $20 \mathrm{~cm}$ intervals. The green regions are $1 \mathrm{~cm}$ thick slabs of polyethylene moderator, each $1 \mathrm{~m}^{2}$ in area.

This comparison of neutron-detection efficiencies only, which relies upon a simple moderated-detector design that has not been subjected to optimization in any of its geometrical parameters, does not purport to establish absolute superiority of the TOF method over moderator-based detection. (For one thing, the responses of the two systems to gamma background have been ignored in this analysis.) Instead, the comparison is presented in an attempt to establish the relevance of the n-TOF method as a legitimate and promising alternative to moderator-based detection, an alternative that should not be dismissed out of hand. This is particularly evident when the potential cost benefits of outfitting an n-TOF sensor, relative to outfitting a moderated ${ }^{3} \mathrm{He}$ tube sensor, are taken into account. The two monolithic plastic scintillator slabs at the heart of the sensor investigated in this project could be purchased for slightly more than $\$ 2,000$ apiece in 2004 . Adding in the price of four PMTs, approximately $\$ 2,500$ total for Hamamatsu 5.08 -cm (2-in.) tubes, yields a total of about $\$ 6,500$ for the detector hardware (without readout electronics) in a hypothetical $n-T O F$ sensor. Comparing this to a price range of $\$ 1,000$ to $\$ 1,500$ for even a single 30-cm-long (not 1-m-long, as assumed in the model) ${ }^{3} \mathrm{He}$ tube, and taking at face value the 5:1 efficiency ratio estimated above from the model of a 5-tube system, one concludes that approximately 25 tubes, at a total cost of anywhere from $\$ 25,000$ to $\$ 38,000$, would be required to outfit a moderatorbased system of comparable neutron detection efficiency. These considerations suggest that the n-TOF approach offers an economical alternative to moderator-based sensors for achieving readily "areascaleable" fast neutron detection coverage in an interrogation system. 


\subsection{References}

Anderson DN, DC Stromswold, SC Wunschel, AJ Peurrung, RR Hansen. 2005. "Detection and Location of Gamma-Ray Sources with a Modulating Coded Mask." To appear in Technometrics.

Craun RL, and DL Smith. 1970. "Analysis of response data for several organic scintillators." Nucl. Instrum. and Meth. 80:239, as cited in (Leo 1987).

Fisher RK, VS Zaveryaev, and SV Trusillo. 1997. "Threshold bubble chamber for measurement of knock-on DT neutron tails from magnetic and inertial confinement experiments." Rev. Sci. Instrum. 68:1103.

Hansen RR, PL Reeder, AJ Peurrung, and DC Stromswold. 2000. "Neutron-gamma discrimination in plastic scintillators.” IEEE Transactions on Nuclear Science. 47:2024.

Jordan DV, JH Ely, AJ Peurrung, LJ Bond, JI Collar, M Flake, MA Knopf, WK Pitts, M Shaver, A Sonnenschein, JE Smart, and LC Todd. 2005. "Neutron detection via bubble chambers." Applied Radiation and Isotopes. In press.

Leo WR. 1987. Techniques for Nuclear and Particle Physics Experiments. New York: Springer-Verlag.

Peurrung AJ. 2000. "Recent developments in neutron detection." Nucl. Instrum. and Meth.A 443:400.

Reeder PL, AJ Peurrung, RR Hansen, and DC Stromswold. 1999. "Detection of fast neutrons in a plastic scintillator using digital pulse processing to reject gammas." Nucl. Instrum. and Meth.A. 422:84.

Reeder PL, DL Stephens, DV Jordan, RC Craig, and B Geelhood. 2003. Progress Report for the Advanced Large-Area Plastic Scintillator (ALPS) Project: FY 2003 Final. PNNL-14490, Pacific Northwest National Laboratory, Richland, WA.

Reilly D, N Ennslin, and H Smith (editors.) 1991. Passive Nondestructive Assay of Nuclear Materials. LA-UR-90-732, prepared for Office of Nuclear Regulatory Research, U.S. Nuclear Regulatory Commission.

Schulze J, W Rosenstock, and HL Kronholz. 1992. "Measurements of fast neutrons by bubble detectors." Rad. Prot. Dosim. 44:351.

Stromswold DC, AJ Peurrung, RR Hansen, and PL Reeder. 1999. Direct Fast-Neutron Detection. PNNL-13068, Pacific Northwest National Laboratory, Richland, WA.

Toyokawa H, A Uritani, C Mori, M Yoshizawa, N Takeda, and K Kudo. 1996. "Neutron spectrometer with position-sensitive proportional counters." Nucl. Instrum. and Meth.A 381:481.

Toyokawa H, M Yoshizawa, A Uritani, C Mori, N Takeda, and K Kudo. 1997. "Performance of a spherical neutron counter for spectroscopy and dosimetry.” IEEE Trans. Nucl. Sci. 44:788. 
Woodring M, D Souza, S Tipnis, P Waer, M Squillante, G Entine, and KP Ziock. 1999. "Advanced radiation imaging of low-intensity gamma-ray sources." Nucl. Instrum. and Meth.A 422:709.

Yamaguchi S, A Uritani, H Sakai, C Mori, T Iguchi, H Toyokawa, N Takeda, and K Kudo. 1999. "Spherical neutron detector for space neutron measurement." Nucl. Instrum. and Meth. A 422:600. 


\section{Distribution}

No. of

Copies

\section{OFFSITE}

1 Los Alamos National Laboratory

P.O. Box 1663

Los Alamos, NM 87545

Attn: M. Abhold

2 Lawrence Livermore National Laboratory P.O. Box 808

Livermore, CA 94551-0808

Attn: D. Dietrich

J. Valentine

1 Oak Ridge National Laboratory

P.O. Box 2008

Oak Ridge, TN 37831

Attn: J. Mihalczo
No. of

Copies

ONSITE

12 Pacific Northwest National Laboratory

M. Cooper

P8-20

D. V. Jordan (5)

P8-20

P. L. Reeder

P8-50

K. R. McCormick

P8-20

A. J. Peurrung

K5-26

W.K. Pitts

P8-01

L.E. Smith

P8-50

G. A. Warren

P8-20

Distr. 1 